\title{
Fixing the quantum three-point function
}

\author{
Yunfeng Jiang, ${ }^{a}$ Ivan Kostov, ${ }^{a}$ Florian Loebbert ${ }^{b, c}$ and Didina Serban ${ }^{a}$ \\ ${ }^{a}$ Institut de Physique Théorique, DSM, CEA, URA2306 CNRS, \\ Saclay, F-91191 Gif-sur-Yvette, France \\ ${ }^{b}$ School of Natural Sciences, Institute for Advanced Study, \\ Einstein Drive, Princeton, NJ 08540, U.S.A. \\ ${ }^{c}$ Niels Bohr International Academy 6 Discovery Center, Niels Bohr Institute, \\ Blegdamsvej 17, 2100 Copenhagen, Denmark \\ E-mail: yunfeng.jiang@cea.fr, ivan.kostov@cea.fr, loebbert@ias.edu, \\ didina.serban@cea.fr
}

ABSTRACT: We propose a new method for the computation of quantum three-point functions for operators in $\mathfrak{s u}(2)$ sectors of $\mathcal{N}=4$ super Yang-Mills theory. The method is based on the existence of a unitary transformation relating inhomogeneous and long-range spin chains. This transformation can be traced back to a combination of boost operators and an inhomogeneous version of Baxter's corner transfer matrix. We reproduce the existing results for the one-loop structure constants in a simplified form and indicate how to use the method at higher loop orders. Then we evaluate the one-loop structure constants in the quasiclassical limit and compare them with the recent strong coupling computation.

KEYWORDS: Supersymmetric gauge theory, Gauge-gravity correspondence, Bethe Ansatz, Integrable Field Theories

ARXIV EPRINT: 1401.0384 


\section{Contents}

1 Introduction $\quad 2$

1.1 The result for the three-point function $\quad 5$

2 Inhomogeneous XXX spin chain $\quad 6$

$\begin{array}{lll}2.1 & \text { Corner transfer matrix } & 9\end{array}$

3 Long-range integrable models $r$

$\begin{array}{lll}3.1 & \text { Boost operators } & 14\end{array}$

4 Map from long-range to inhomogeneous models $\quad 17$

$\begin{array}{lll}4.1 & \text { S-operator } & 18\end{array}$

4.2 Morphism property and scalar products 21

4.3 Morphism and theta-morphism 24

5 Three-point function of $\mathfrak{s u ( 2 )}$ fields beyond tree level 26

$\begin{array}{lll}5.1 & \text { Three-point functions at one loop } & 27\end{array}$

6 Three-point functions in the semi-classical limit 31

6.1 Scalar products and norms in the semi-classical limit 32

6.2 One-loop three-point function in the semi-classical limit 33

6.3 Comparison with the string theory results 35

7 Conclusions \& outlook $\quad 39$

A Inhomogeneous CTM at Order $\theta^{3} \quad 40$

B The BDS charges from boost deformations $\quad 40$

C Derivation of the S-operator at order $g^{2} \quad 41$

D From permutations to derivatives 442

D.1 PD relations in the bulk $\quad 42$

D.2 PD relations at the boundary 44

E From S-transformation to theta-morphism 44

$\begin{array}{llr}\text { F Reduction formula } & 48\end{array}$

G Calculation of three-point function $\quad 49$ 


\section{Introduction}

Integrability has already proven to be a powerful tool for finding a solution to the spectral problem of supersymmetric gauge theories (see e.g. [1]), and to test their duality to string theories [2]. In the last few years, the applications of integrability methods were largely extended to other fundamental objects in gauge theory, such as scattering amplitudes or Wilson loops (see e.g. [3-18]) as well as to correlation functions. The majority of these computations was concerned with $\mathcal{N}=4$ super Yang-Mills (SYM) theory which is also subject to this work.

The first computations of correlation functions were performed in the early days of the AdS/CFT correspondence for protected BPS operators [19, 20]. For non-protected operators at weak coupling, progress was made using the map to spin chains [21-23]. The most advanced results concerning "heavy" operators, i.e. operators with large $R$-charge, were obtained at tree-level and in the $\mathfrak{s u}(2)$ sector [23-26], but results for the $\mathfrak{s u}(3)$ [27-29] and $\mathfrak{s l}(2)[30-33]$ sectors are also available. To extend the computation of structure constants to higher loops, one needs as a crucial input the field-theoretical computation of loop corrections to the three-point function [22,34-36]. Results at loop order were obtained for the $\mathfrak{s u}(2)$ sector in [37-39] using the spin chain technology, and in [40] using the coherent state representation and the Landau-Lifshitz model. At strong coupling, an important effort was invested in formulating the problem and in computing special configurations of three-point functions, both using integrability methods [41-46] and string techniques [47-54]. Here the conformal bootstrap was also successfully applied [55-57].

Each of these results covers a particular case of three- (or higher-) point functions, and we do not yet have a comprehensive understanding of the generic structure of correlation functions, as we do for the spectrum. In particular, we do not yet have a method which provides an acceptable recipe for obtaining a particular three-point function. Nevertheless, a coherent picture starts to emerge, and an important step forward is the very recent calculation of $\mathfrak{s u}(2)$ correlation functions by Kazama and Komatsu at strong coupling [46].

In this work, we revisit the computation of quantum three-point functions in [37-39], with the purpose 1) to get reliable expressions in the semi-classical limit, which can be compared to the strong coupling results and 2) to set up a systematic formalism for proceeding to higher loop orders. In order to extend the results from tree-level to loops, we need to have a good description of the wave functions and scalar products of long-range interacting spin chains.

With this motivation in mind, we study a method of generating long-range deformations of nearest-neighbor spin chains. Here we consider the case of the XXX spin chain with spin equal to $1 / 2$, since it is directly applicable to the computation of correlation functions in the $\mathfrak{s u}(2)$ sector of $\mathcal{N}=4$ super Yang-Mills theory. As a prototype of long-range deformation, we consider the BDS model proposed by Beisert, Dippel and Staudacher [58], which was shown [59] to be equivalent to a spin-sector reduction of the one-dimensional Hubbard model at half-filling. The method we use here is very general and it encompasses a large class of deformations. 
Several different methods were employed to describe and solve long-range spin chains, at least partially. Historically, one of the first methods to completely solve a long-range system is based on so-called Dunkl operators [60], and it was used successfully for the HaldaneShastry model, and for some aspects of the infinite length Inozemtsev model [38, 61]. The drawback of this method is that an explicit representation of the Dunkl operators is known only for a restricted class of models. Another restriction is that, with the exception of the Haldane-Shastry model, the Dunkl operators cannot be rendered periodic on a finite lattice. The price to pay for rendering the lattice finite is to introduce a defect [62]. The advantage is that explicit exact expressions for the monodromy matrix can be obtained, and the scalar products are relatively straightforward to compute [38]. Another method to deform the XXX spin chain uses so-called boost and bilocal charges and was proposed in $[63,64]$. This method works again fairly well for long spin chains, but does not include wrapping interactions.

Here, we use yet another method, which is to map the inhomogeneous XXX model to a long-range model. The authors of [58] noticed that the spectral equations, (i.e. the Bethe ansatz equations) of the long-range model they have proposed, can be obtained from those of an inhomogeneous spin chain by carefully choosing the values of the inhomogeneities. This equivalence ceases to hold when wrapping interactions, i.e. interactions of range equal or greater than the length of the spin chain, are taken into account. However, the Hamiltonian of the inhomogeneous spin chain is not a homogeneous long-range spin chain, because it depends on inhomogeneities, which are site-dependent. The observation of BDS was taken further in [64], where it was noticed that if the two spin chains have the same spectrum, then they should be related by a unitary transformation, which was computed up to two-impurity order (or two-loop order in $\mathcal{N}=4 \mathrm{SYM}$ terms). This unitary operator was not explicitly used before to construct the eigenfunctions of the long-range spin chain. Instead, the wave functions of long-range spin chains were constructed via another relation to inhomogeneous spin chains [37, 39] or by the relation to Dunkl operators [38, 62].

In this paper we elaborate on the observation by Bargheer, Beisert and one of the authors [64] and give a systematic method to construct eigenvectors and scalar products of the BDS model which are exact up to wrapping order. We emphasize that we consider the periodic model. The computation of scalar products is straightforward if the existence of the unitary similarity transformation (the S-operator) from the inhomogeneous to the long-range spin chain is assumed. The method is general and it applies to all spin chains that can be obtained perturbatively with boost deformations from the XXX model. To compute explicitly the wave functions, one needs the explicit expression of the unitary transformation S, which we derive here up to quadratic order in the inhomogeneities. ${ }^{1}$ We find that the unitary transformation can be constructed using the long-range boost deformations and an inhomogeneous version of Baxter's corner transfer matrix:

$$
\begin{array}{ccl}
\text { Inhomogeneous spin chain } & \text { S-operator } & \text { Homogenous long-range spin chain } \\
\text { with inhomogeneities } \theta_{k} & \longleftrightarrow & \text { with coupling constants } \sigma_{n}=\sum_{k} \theta_{k}^{n}
\end{array}
$$

\footnotetext{
${ }^{1}$ For the specific BDS inhomogeneities this unitary transformation was already given in [64].
} 
For verification, we demonstrate that applying the following two operations, reproduces the differential operator found in $[37,39]$ plus the required boundary terms: 1) shifting the inhomogeneities from zero to their non-zero (e.g. BDS-like) values and 2) applying the unitary transformation $\mathrm{S}$ which transforms the chain to a homogeneous long-range chain. The procedure, although relatively tedious, is straightforward and can be applied at higher orders. It furthermore proves the conjectures and observations on the all-loop norms (without dressing phase) in [37, 39]. The results are relatively simple and elegant, due to the manifest structure of the transformations.

In the next step, we apply the method described above to the computation of threepoint correlation functions in the $\mathfrak{s u}(2)$ sector of $\mathcal{N}=4$ SYM theory in the planar limit. The key property that we use is the freedom to choose the values of the inhomogeneities, as long as their symmetric sums $\sigma_{n}$ are kept at the model-specific values. This can be done in perturbation theory for sufficiently large chains. The results for the three-point function are summarized in section 1.1.

Our result resembles the asymptotic solution of the spectral problem in the $\mathfrak{s u}(2)$ sector, where fixing the inhomogeneities in the Bethe ansatz to the BDS values was enough to obtain the long-range Bethe ansatz encoding the higher loop spectrum. Here we get the long-range three-point function in a similar way: we take the inhomogeneous three-point vertex, and after fixing the inhomogeneities, we add a correction given by an operator that acts merely on the splitting points of the involved spin chains.

The result is a very concise expression for the structure constant in terms of the rapidities of the three states. An attractive property of this expression is that it allows to obtain without pain the semiclassical limit of three heavy operators. We computed the quasiclassical limit of the one-loop structure constant and compared it with the Frolov-Tseytlin limit of the result of [46]. Both expressions are given by contour integrals of dilogarithm functions, up to terms that vanish in the Frolov-Tseytlin limit. In the Frolov-Tseytlin limit the insertions at the splitting points are of subleading order, and the gauge theory result is given by the inhomogeneous three-point vertex, after fixing the inhomogeneities. We find that the integrands match, which is already a strong evidence that the correspondence with the string theory persists at one loop. Moreover, we reveal through this comparison the reason for the asymmetric form of the gauge theory structure constant, while the string theory result is completely symmetric with respect to permutations of the three operators. To complete the result one should also compare the integration contours. This is a subtle issue which is still lacking complete understanding, both in the gauge and in the string theory. At the present stage the contours of integration are chosen case by case by taking into account the analytic properties of the solution.

The structure of the paper is the following: in section 2 we remind of the definition of the inhomogeneous XXX spin chain and we define its conserved charges. In section 2.1 we define the corner transfer matrix (CTM) and its inhomogeneous version and we remind of the link between the CTM and the (first) boost operator. Section 3 is devoted to longrange spin chains, including the BDS spin chain, and more generally to the local boost deformations of the XXX Hamiltonian. In section 4, we make explicit the map between the local boost deformations and inhomogeneous spin chains, by defining the operator $\mathrm{S}$ and 
determining it to order $g^{2}$. We compare with the result obtained from the CTM and then compute the scalar products up to wrapping order. We also explore the morphism of the Yangian algebra defined by the operator $\mathrm{S}$ and we derive the action of this morphism on the elements of the monodromy matrix and on the Bethe vectors. In section 5 we show how to compute the three-point function at one-loop order. In section 6 we take the semiclassical limit of the one-loop expression and compare it with the Frolov-Tseytlin limit of [46].

\subsection{The result for the three-point function}

In this section we summarize our results for the three-point function of operators in different $\mathfrak{s u}(2)$ sectors taking the generic form

$$
\left\langle\mathcal{O}^{(1)}\left(x_{1}\right) \mathcal{O}^{(2)}\left(x_{2}\right) \mathcal{O}^{(3)}\left(x_{3}\right)\right\rangle=\frac{N_{c}^{-1} \sqrt{L^{(1)} L^{(2)} L^{(3)}} C_{123}\left(g^{2}\right)}{\left|x_{12}\right|^{\Delta^{(1)}+\Delta^{(2)}-\Delta^{(3)}}\left|x_{13}\right|^{\Delta^{(1)}+\Delta^{(3)}-\Delta^{(2)}}\left|x_{23}\right|^{\Delta^{(2)}-\Delta^{(3)}-\Delta^{(1)}}} .
$$

The operators are chosen such that they have definite conformal dimensions $\Delta^{(1)}, \Delta^{(2)}$ and $\Delta^{(3)}$, and belong to three different $\mathfrak{s u}(2)$ sectors

$$
\mathcal{O}^{(1)} \in\{Z, X\}, \quad \mathcal{O}^{(2)} \in\{\bar{Z}, \bar{X}\}, \quad \mathcal{O}^{(3)} \in\{Z, \bar{X}\} .
$$

In the language of spin chains, they are characterized by three Bethe vectors $\left|\mathbf{u}^{(1)}\right\rangle,\left|\mathbf{u}^{(2)}\right\rangle$, and $\left|\mathbf{u}^{(3)}\right\rangle$ with lengths $L^{(1)}, L^{(2)}$ and $L^{(3)}$, respectively. By $\mathbf{u}^{(a)}$ we denote the set of the magnon rapidities $\left\{u_{1}^{(a)}, \ldots, u_{N^{(a)}}^{(a)}\right\}$. The renormalization scheme invariant part $C_{123}\left(g^{2}\right)$ can be expressed in the spin-chain language as [21, 22]

$$
C_{123}\left(g^{2}\right)=\frac{\left\langle\mathbf{u}^{(1)}, \mathbf{u}^{(2)}, \mathbf{u}^{(3)}\right\rangle}{\left(\left\langle\mathbf{u}^{(1)} \mid \mathbf{u}^{(1)}\right\rangle\left\langle\mathbf{u}^{(2)} \mid \mathbf{u}^{(2)}\right\rangle\left\langle\mathbf{u}^{(3)} \mid \mathbf{u}^{(3)}\right\rangle\right)^{1 / 2}} .
$$

In the expression above, $\left\langle\mathbf{u}^{(a)} \mid \mathbf{u}^{(a)}\right\rangle$ are the square norms of the Bethe vectors, which were evaluated in $[23,26]$ using the Gaudin-Korepin formula $[65,66]$. Our result for the threepoint function concerns the loop expression of the cubic vertex

$$
\left\langle\mathbf{u}^{(1)}, \mathbf{u}^{(2)}, \mathbf{u}^{(3)}\right\rangle=\left(1+g^{2} \hat{\Delta}_{21}+\mathcal{O}\left(g^{4}\right)\right) \mathscr{A}_{\mathbf{u}^{(1)} \cup \mathbf{u}^{(2)}, \boldsymbol{\theta}^{(12)}}\left(1+g^{2} \hat{\Delta}_{03}+\mathcal{O}\left(g^{4}\right)\right) \mathscr{A}_{\mathbf{u}^{(3)}, \boldsymbol{\theta}^{(13)}} .
$$

Above, the functional $\mathscr{A}_{\mathbf{u}, \boldsymbol{\theta}}$ is expressible in terms of a determinant, see section 4.2 , where the set of inhomogeneities $\boldsymbol{\theta}^{(a b)}$ is given by the BDS-like values $[58,59]$

$$
\theta_{l}^{(a b)}=2 g \sin \frac{2 \pi l}{L^{(a b)}}, \quad l=1, \ldots, L^{(a b)}, \quad 2 L^{(a b)}=L^{(a)}+L^{(b)}-L^{(c)} .
$$

It gives the main contribution to the loop-order three-point function and captures the main effect of the mixing of operators at loop order, at least for heavy operators. The operators $\hat{\Delta}_{21}$ and $\hat{\Delta}_{03}$ in (1.4) compute the effect of the insertions [22, 34] and of the mixing near the splitting points. The operators $\hat{\Delta}_{a b}$ act as follows (by convention we take the vacuum to be the state $\left|\mathbf{u}^{(0)}=\emptyset\right\rangle=|\Omega\rangle$ )

$$
\hat{\Delta}_{a b} \mathscr{A}_{\mathbf{u}^{(a)} \cup \mathbf{u}^{(b)}, \boldsymbol{\theta}^{(b c)}}=\left.\left(\partial_{1}^{(b)} \partial_{2}^{(b)}-i \delta \mathrm{E}_{2} \partial_{1}^{(b)}+i \delta \mathrm{E}_{3}-\frac{1}{2} \delta \mathrm{E}_{2}^{2}\right) \mathscr{A}_{\mathbf{u}^{(a)} \cup \mathbf{u}^{(b)}, \boldsymbol{\theta}^{(b c)}}\right|_{\theta=0},
$$


with $\partial_{j}^{(a)} \equiv \partial / \partial \theta_{j}^{(a)}$ and $\delta \mathrm{E}_{r}=\mathrm{E}_{r}^{(b)}-\mathrm{E}_{r}^{(a)}$ being the difference of the conserved charges between the ket and bra states.

We obtained the quasiclassical limit of (1.4) and compare it with the FrolovTseytlin [67] limit ${ }^{2}$ of the strong coupling result of [46]. In the quasiclassical limit the roots from the set $\mathbf{u}^{(a)}$ condense into one or several cuts (describing macroscopic Bethe strings) and the state $\left|\mathbf{u}^{(a)}\right\rangle$ is characterised by its quasimomentum $p^{(a)}$, which has discontinuities across the cuts. Up to terms that can be neglected in the Frolov-Tseytlin limit, ${ }^{3}$ the logarithm of the structure constant is given by the contour integral

$$
\begin{aligned}
\log C_{123}(g) & \simeq \oint_{\mathcal{C}^{(12 \mid 3)}} \frac{d u}{2 \pi} \operatorname{Li}_{2}\left(e^{i p^{(1)}(u)+i p^{(2)}(u)-i q^{(3)}(u)}\right) \\
& +\oint_{\mathcal{C}^{(13 \mid 2)}} \frac{d u}{2 \pi} \operatorname{Li}_{2}\left(e^{i p^{(3)}(u)+i q^{(1)}(u)-i q^{(2)}(u)}\right)-\frac{1}{2} \sum_{a=1}^{3} \int_{\mathcal{C}^{(a)}} \frac{d z}{2 \pi} \operatorname{Li}_{2}\left(e^{2 i p^{(a)}(z)}\right) .
\end{aligned}
$$

The integration contours should be placed taking into account the analytical properties of the integrand. The three quasimomenta $p^{(a)}$ depend on $g$ through the distribution of the inhomogeneities. The functions $q^{(a)}$ are obtained from $p^{(a)}$ by subtracting the resolvent for the Bethe roots $\mathbf{u}^{(a)}$. Assuming that the contours of integration are the same, the difference between (1.6) and the contour integral obtained in [46] resides in the integrand. ${ }^{4}$ In this paper we show that the integrand of (1.6) coincides with the linear order in $g^{2}$ of the expansion of the integrand in the string solution. The comparison shows that the asymmetry of the integrand in (1.6) in the three quasimomenta is a consequence of the specific choice of the three $\mathfrak{s u}(2)$ sectors in $\mathfrak{s o}(4)$ used in the weak coupling computation.

\section{Inhomogeneous XXX spin chain}

In this section we gather some well-known facts about the (periodic) inhomogeneous XXX spin chain. It is is defined by the expression of its monodromy matrix

$$
\mathrm{M}_{\alpha}(u ; \theta)=\prod_{k=1}^{L} \mathrm{R}_{\alpha k}\left(u-\theta_{k}-\frac{i}{2}\right),
$$

where the rational R-matrix takes the form ${ }^{5}$

$$
\mathrm{R}_{\alpha \beta}(u)=\frac{u}{u+i} \mathrm{I}_{\alpha \beta}+\frac{i}{u+i} \mathrm{P}_{\alpha \beta} .
$$

\footnotetext{
${ }^{2}$ If we introduce a scale for the lengths, with $L^{(a)} \rightarrow \infty, L^{(a)} / L$ and $N^{(a)} / L$ finite, then the FrolovTseytlin limit means that $g / L \ll 1$.

${ }^{3}$ The subleading terms are the contributions of the operators $\hat{\Delta}_{a b}$.

${ }^{4}$ Also, there are certain terms that vanish by kinematical reasons in the gauge theory computation and which do not seem to vanish in the string theory computation. We believe that this issue will be resolved soon.

${ }^{5}$ This normalization for the $\mathrm{R}$ matrix is convenient for obtaining the good conserved quantities, however, for constructing the eigenvectors we find it more convenient to use the normalization $\mathrm{R}^{\prime}=\mathrm{I}+i \mathrm{P} / u$.
} 
and the operator $\mathrm{P}_{\alpha \beta}$ represents a permutation of the spins in the spaces $\alpha$ and $\beta$. The monodromy matrix $\mathrm{M}_{\alpha}(u ; \theta)$ obeys the Yang-Baxter equation ${ }^{6}$

$$
\mathrm{R}_{\alpha \alpha^{\prime}}(u-v) \mathrm{M}_{\alpha}(u) \mathrm{M}_{\alpha^{\prime}}(v)=\mathrm{M}_{\alpha^{\prime}}(v) \mathrm{M}_{\alpha}(u) \mathrm{R}_{\alpha \alpha^{\prime}}(u-v) .
$$

When the inhomogeneities $\theta_{k}$ are set to zero, or they are all equal to each other, this is the usual homogeneous XXX spin chain. The inhomogeneities can be interpreted as some extra degrees of freedom which have been frozen. It will be convenient to write the monodromy matrix in the auxiliary space denoted by the index $\alpha$ :

$$
\mathrm{M}_{\alpha}(u)=\left(\begin{array}{ll}
\mathrm{A}(u) & \mathrm{B}(u) \\
\mathrm{C}(u) & \mathrm{D}(u)
\end{array}\right)_{\alpha}
$$

As for the homogeneous spin chain, the transfer matrix

$$
\mathrm{T}(u)=\operatorname{Tr}_{\alpha} \mathrm{M}_{\alpha}(u)=\mathrm{A}(u)+\mathrm{D}(u)
$$

commutes with itself for any value of the spectral parameter (i.e. $[T(u), T(v)]=0$ ) and it therefore generates the integrals of motion.

Since $\mathrm{M}(u)$ obeys the Yang-Baxter equation with the rational R-matrix $\mathrm{R}(u)=$ $(u+i \mathrm{P}) /(u+i)$ the algebra of the matrix elements is the same as for the homogeneous XXX model:

$$
\begin{aligned}
\mathrm{A}(v) \mathrm{B}(u) & =\frac{u-v+i}{u-v} \mathrm{~B}(u) \mathrm{A}(v)-\frac{i}{u-v} \mathrm{~B}(v) \mathrm{A}(u), \\
\mathrm{D}(v) \mathrm{B}(u) & =\frac{u-v-i}{u-v} \mathrm{~B}(u) \mathrm{D}(v)+\frac{i}{u-v} \mathrm{~B}(v) \mathrm{D}(u) .
\end{aligned}
$$

The Hilbert space is spanned by states obtained from the pseudo vacuum $|\Omega\rangle=|\uparrow \uparrow \ldots \uparrow\rangle$ by acting with the "raising operators" $\mathrm{B}(u)$ :

$$
|\mathbf{u}\rangle=\mathrm{B}\left(u_{1}\right) \ldots \mathrm{B}\left(u_{M}\right)|\Omega\rangle .
$$

If the rapidities $\mathbf{u}=\left\{u_{1}, \ldots, u_{M}\right\}$ are generic, the state is called "off-shell", and the state is called "on-shell" if the rapidities obey the Bethe ansatz equations

$$
\frac{a\left(u_{j}\right)}{d\left(u_{j}\right)}=\prod_{\substack{k=1 \\ k \neq j}}^{M} \frac{u_{j}-u_{k}+i}{u_{j}-u_{k}-i}
$$

where $a(u)$ and $d(u)$ are the eigenvalues of the diagonal operators $\mathrm{A}(u)$ and $D(u)$ :

$$
a(u)=1 \quad d(u)=\prod_{l=1}^{L} \frac{\left(u-\frac{i}{2}-\theta_{l}\right)}{\left(u+\frac{i}{2}-\theta_{l}\right)} .
$$

\footnotetext{
${ }^{6}$ In the following we will skip the variables $\theta=\left\{\theta_{1}, \ldots, \theta_{L}\right\}$ from the notations, since the algebraic relations are generic. To denote the homogeneous (short-range) quantities we will use the index [0] or SR.
} 
The "on-shell" states are eigenstates of the transfer matrix $\mathrm{T}(u)$ with the eigenvalue

$$
t(u)=\frac{Q(u-i)}{Q(u)}+\frac{d(u)}{a(u)} \frac{Q(u+i)}{Q(u)}, \quad \text { with } \quad Q(u)=\prod_{k=1}^{M}\left(u-u_{k}\right) .
$$

We define the integrals of motion of the inhomogeneous spin chain model conventionally as the logarithmic derivatives of the transfer matrix $\mathrm{T}(u)$ around the point $u=i / 2$ :

$$
\mathrm{Q}_{r}^{\theta}=\left.\frac{1}{i(r-1) !} \frac{d^{r-1}}{d u^{r-1}} \ln \mathrm{T}(u)\right|_{u=i / 2} .
$$

Any combination of the above integrals of motion is an integral of motion and we are going to use later this property in order to define a more convenient basis of charges. The definition given above is convenient if the values of the inhomogeneities are small, $\theta_{k} \sim g$, where $g$ is a perturbation parameter which will be specified later. It extends the definition of the homogeneous case (i.e. $\theta_{k}=0$ ), for which the first conserved quantity is the shift operator:

$$
\mathrm{U}_{0} \equiv \mathrm{T}_{0}\left(\frac{i}{2}\right)=\operatorname{Tr}_{\alpha} \prod_{k=1}^{L} \mathrm{P}_{\alpha k}=\mathrm{P}_{L-1, L} \mathrm{P}_{L-2, L-1} \ldots \mathrm{P}_{12} .
$$

The homogeneous shift $\mathrm{U}_{0}$ translates the chain by one lattice spacing, that is we have

$$
\mathrm{U}_{0} \mathrm{P}_{k, k+1} \mathrm{U}_{0}^{-1}=\mathrm{P}_{k-1, k} .
$$

Periodicity of the chain means that $\mathrm{U}_{0}^{L}=1$. The first homogeneous Hamiltonians take the form

$$
\mathrm{Q}_{2}^{\mathrm{SR}}=\sum_{k=1}^{L} \mathrm{H}_{k}, \quad 2 \mathrm{Q}_{3}^{\mathrm{SR}}=i \sum_{k=1}^{L}[\mathrm{H}]_{k-1}, \quad 3 \mathrm{Q}_{4}^{\mathrm{SR}}=\sum_{k=1}^{L}\left([[\mathrm{H}]]_{k-1}+\mathrm{H}_{k}[\mathrm{H}]_{k-1}-[\mathrm{H}]_{k-1}-\mathrm{H}_{k}\right)
$$

where we have introduced the compact recursive notation

$$
\mathrm{H}_{k}=\mathrm{I}_{k, k+1}-\mathrm{P}_{k, k+1}, \quad[\mathrm{H}]_{k}=\left[\mathrm{H}_{k}, \mathrm{H}_{k+1}\right], \quad[[\mathrm{H}]]_{k}=\left[[\mathrm{H}]_{k}, \mathrm{H}_{k+2}\right] .
$$

For completeness we note that in terms of the R-matrix the nearest-neighbor Hamiltonian is given by

$$
\mathrm{H}_{k}=-\left.i \mathrm{R}_{k, k+1}^{-1}(u) \frac{d \mathrm{R}_{k, k+1}(u)}{d u}\right|_{u=0},
$$

and the homogeneous transfer matrix can be expressed in the convenient form

$$
\mathrm{T}_{0}\left(u+\frac{i}{2}\right)=\mathrm{U}_{0} \exp \left[i \sum_{r=2}^{L} u^{r-1} \mathrm{Q}_{r}^{\mathrm{SR}}\right] .
$$

In the inhomogeneous case the conserved quantities do not take the simple form (2.14), but it is useful for later purposes to write them as an expansion in the value of the inhomogeneities. The momentum is no longer a conserved quantity, since the inhomogeneous 
chain is not translationally invariant. However, the periodicity condition $\mathrm{U}_{0}^{L}=1$ still holds. The conserved quantity which replaces the shift $\mathrm{U}_{0}$ is the operator

$$
\mathrm{U}_{\theta}=\operatorname{Tr}_{\alpha} \prod_{k=1}^{L} \mathrm{R}_{\alpha k}\left(-\theta_{k}\right)
$$

whose expansion in $\theta$ exponentiates to ${ }^{7}$

$$
\mathrm{U}_{\theta}=\mathrm{U}_{0} \exp \left[-i \sum_{k} \theta_{k} \mathrm{H}_{k}-\frac{1}{2} \sum_{k} \theta_{k-1} \theta_{k}[\mathrm{H}]_{k-1}+\mathcal{O}\left(\theta^{2}\right)\right] .
$$

Note that for $\theta_{k}=-u$ the inhomogeneous shift $\mathrm{U}_{\theta}$ gives back the homogeneous transfer matrix $\mathrm{T}_{0}(u+i / 2)(2.17)$. The expansion of the inhomogeneous Hamiltonian takes the form

$$
\mathrm{Q}_{2}^{\theta}=\sum_{k=1}^{L}\left[\mathrm{H}_{k}-i \theta_{k}[\mathrm{H}]_{k-1}+\theta_{k}^{2}\left(\mathrm{H}_{k}[\mathrm{H}]_{k-1}-[\mathrm{H}]_{k-1}-\mathrm{H}_{k}\right)+\theta_{k} \theta_{k+1}[[\mathrm{H}]]_{k-1}\right]+\mathcal{O}\left(\theta^{3}\right)
$$

The $M$-magnon eigenvalues $E_{r}$ of the conserved quantities $\mathrm{Q}_{r}$ are the sum over one-magnon eigenvalues

$$
E_{r}^{\theta}=\sum_{j=1}^{M} q_{r}\left(u_{j}\right)+\mathcal{O}\left(\theta^{L}\right)
$$

where $q_{r}(u)$ takes the standard form of the XXX one-magnon eigenvalues

$$
q_{r}(u)=\frac{i}{r-1}\left(\frac{1}{\left(u+\frac{i}{2}\right)^{r-1}}-\frac{1}{\left(u-\frac{i}{2}\right)^{r-1}}\right) .
$$

Here $u_{1}, \ldots, u_{M}$ are solutions of the Bethe ansatz equations (2.8) and as such they depend on the values of the inhomogeneities $\theta_{k}$.

\subsection{Corner transfer matrix}

An interesting quantity with regard to the construction of integrals of motion is Baxter's corner transfer matrix (CTM) [68]. After a brief review of some aspects of the CTM for homogeneous spin chains, we define an inhomogeneous CTM that will be useful in the subsequent sections.

Homogeneous chains. Let us briefly review the definition of a homogeneous CTM and its relation to the so-called nearest-neighbor boost operator, cf. [68-70]. In the following we will assume to work on infinite chains $(L \rightarrow \infty)$ or in the bulk of a periodic chain, respectively. ${ }^{8}$ First we introduce a half-row matrix $\mathrm{G}_{A}$ ranging from site $A(<L)$ to site $L$ :

$$
\mathrm{G}_{A}(u)=\hat{\mathrm{R}}_{L-1, L}(u) \hat{\mathrm{R}}_{L-2, L-1}(u) \ldots \hat{\mathrm{R}}_{A+1, A+2}(u) \hat{\mathrm{R}}_{A, A+1}(u) .
$$

\footnotetext{
${ }^{7}$ We assume periodic boundary conditions, $k+L \equiv k$.

${ }^{8}$ Note that typically some spins on the edge of the CTM are fixed.
} 


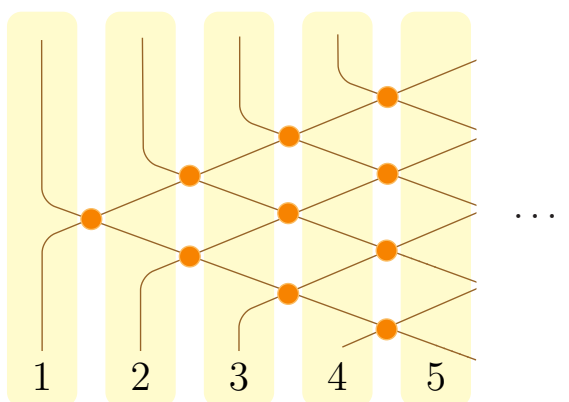

Figure 1. Corner transfer matrix (CTM) acting on a spin chain. We assume an infinite lattice on the right hand side. The small discs denote $\hat{\mathrm{R}}$-matrices defined in (2.24).

Here we have defined the symbol $\hat{\mathrm{R}}(u)$ as the R-matrix times the permutation operator: ${ }^{9}$

$$
\hat{\mathrm{R}}_{k, k+1}(u)=\mathrm{P}_{k, k+1} \mathrm{R}_{k, k+1}(u) .
$$

Then we define the CTM as a stack of half-row matrices of different lengths according to (cf. figure 1)

$$
\mathcal{A}(u)=\mathrm{G}_{1}(u) \ldots \mathrm{G}_{L-2}(u) \mathrm{G}_{L-1}(u) .
$$

Note that the triangular definition of the CTM originates in the context of vertex models. In fact, this matrix can be defined for every quadrant of a square lattice of R-matrices (vertices). In the bulk the half-row matrix $\mathrm{G}_{A}$ has (up to the shift) the same structure as the parity inverted row-to-row transfer matrix $\mathrm{T}^{-1}\left(-u+\frac{i}{2}\right)$ and consequently a similar expansion $^{10}$

$$
\mathrm{G}_{A}(u)=1+i u \sum_{k=A}^{L} \mathrm{H}_{k}+\mathcal{O}\left(u^{2}\right) .
$$

This form makes it clear that the CTM expands as

$$
\mathcal{A}(u)=1+i u \mathcal{B}\left[\mathrm{Q}_{2}^{\mathrm{SR}}\right]+\mathcal{O}(u)^{2},
$$

where $\mathcal{B}\left[\mathrm{Q}_{2}^{\mathrm{SR}}\right]$ denotes the so-called boost operator of the nearest-neighbor Hamiltonian $\mathrm{Q}_{2}^{\mathrm{SR}}=\sum_{k} \mathrm{H}_{k}$. For a generic local operator $\mathcal{L}$ with local density $\mathcal{L}_{k}$, the boost is defined as

$$
\mathcal{B}[\mathcal{L}]=\sum_{k} k \mathcal{L}_{k}
$$

It is well-known that the boost of the nearest-neighbor Hamiltonian allows to obtain higher integrable Hamiltonians of a short-range spin chain model based on a rational (or trigonometric) R-matrix [71]:

$$
\mathrm{Q}_{r+1}^{\mathrm{SR}}=-\frac{i}{r}\left[\mathcal{B}\left[\mathrm{Q}_{2}^{\mathrm{SR}}\right], \mathrm{Q}_{r}^{\mathrm{SR}}\right]
$$

\footnotetext{
${ }^{9}$ Usually the CTM is defined in terms of ordinary R-matrices or vertex weights and the ingoing site $k$ is identified with the outgoing site $k+1$ when mapping the vertex model to a spin chain. Here it seems convenient to circumvent the vertex model interpretation to avoid confusion.

${ }^{10}$ Note that $\mathrm{R}^{-1}(u)=\mathrm{R}(-u)$.
} 
In fact, on infinite chains the homogeneous CTM can be expressed as the exponential of the nearest-neighbor boost operator as shown in $[68,69]$ for the XYZ model:

$$
\mathcal{A}(u)=\exp \left(i u \mathcal{B}\left[\mathrm{Q}_{2}^{\mathrm{SR}}\right]\right) .
$$

Since the row-to-row transfer matrix $\mathrm{T}(u)$ is the generating function of the local integrals of motion, (2.29) is equivalent to the differential equation $[69,72]$

$$
\frac{d}{d u} \mathrm{~T}\left(u+\frac{i}{2}\right)=i\left[\mathcal{B}\left[\mathrm{Q}_{2}^{\mathrm{SR}}\right], \mathrm{T}\left(u+\frac{i}{2}\right)\right], \quad \mathrm{T}\left(\frac{i}{2}\right)=\mathrm{U}_{0},
$$

where we have fixed the initial value of the transfer matrix to be the homogeneous shift operator. This implies that a finite boost transformation corresponds to a shift of the rapidity parameter of the row-to-row transfer matrix:

$$
\mathcal{A}^{-1}(u) \mathrm{T}(v) \mathcal{A}(u)=\mathrm{T}(u+v) .
$$

In particular, one can understand the row-to-row transfer matrix as being generated by the CTM through a transformation of the shift operator $\mathrm{U}_{0}=\mathrm{T}(i / 2)$ :

$$
\mathrm{T}\left(u+\frac{i}{2}\right)=\mathcal{A}^{-1}(u) \mathrm{U}_{0} \mathcal{A}(u) .
$$

Inhomogeneous chains. Now we would like to extend the above considerations to inhomogeneous spin chains. We define the inhomogeneous CTM as a stack of homogeneous half-row matrices with different rapidity shifts: ${ }^{11}$

$$
\mathcal{A}_{\theta}(u)=\mathrm{G}_{1}\left(u-\theta_{1}\right) \mathrm{G}_{2}\left(u-\theta_{2}\right) \ldots \mathrm{G}_{L}\left(u-\theta_{L}\right) .
$$

Expanding this inhomogeneous CTM evaluated at $u=0$ in terms of the inhomogeneities $\theta$ we find ${ }^{12}$

$$
\mathcal{A}_{\theta}(0)=\exp \left[i \sum_{k} \nu_{k} \mathrm{H}_{k}-\frac{1}{2} \sum_{k} \hat{\rho}_{k}[\mathrm{H}]_{k-1}+\mathcal{O}\left(\theta^{3}\right)\right] .
$$

where the coefficients $\nu_{k}$ and $\hat{\rho}_{k}$ are given by

$$
\nu_{k}=-\sum_{x=1}^{k} \theta_{x}, \quad \hat{\rho}_{k}=-\theta_{k} \nu_{k}-\sum_{x=1}^{k} \theta_{x}^{2} .
$$

In analogy to (2.33) we may interpret the inhomogeneous shift operator as being generated by the operator $\mathcal{A}_{\theta}$ on infinite chains:

$$
\mathrm{U}_{\theta}=\mathcal{A}_{\theta}^{-1}(0) \mathrm{U}_{0} \mathcal{A}_{\theta}(0) .
$$

While we have no proof for this transformation property in general, we have verified it up to order $g^{2}$. Similarly one can check that the inhomogeneous bulk Hamiltonian is generated according to $\mathrm{Q}_{2}^{\theta}=\mathcal{A}_{\theta}^{-1}(0) \mathrm{Q}_{2}^{\mathrm{SR}} \mathcal{A}_{\theta}(0)$, at least up to order $g^{2}$. In section 4 we will rediscover the inhomogeneous CTM in the context of a map between inhomogeneous and long-range spin chains. It would be very interesting to investigate in greater detail how this CTM generates the asymptotic inhomogeneous spin chain model from an ordinary short-range chain.

\footnotetext{
${ }^{11}$ In [73] it was speculated on the connection of the long-range deformations discussed in the subsequent sections to an inhomogeneous version of the CTM. We have not found any discussion of the inhomogeneous CTM defined in (2.34) in the literature.

${ }^{12}$ For an expansion of the inhomogeneous CTM at order $\theta^{3}$ see appendix A.
} 


\section{Long-range integrable models}

By deforming the homogeneous short-range XXX model, one can obtain long-range spin chain models. One possibility is to define these models exactly, for any value of the deformation parameter and for any length of the chain. For example this is the case for the Inozemtsev model [74] whose Hamiltonian takes the form

$$
\mathrm{H}_{\mathrm{I}}=\prod_{\substack{k=1 \\ k \neq l}}^{L} \mathcal{P}_{L, i \pi / \kappa}(k-l) \mathrm{P}_{k l} .
$$

Here $\mathcal{P}_{L, i \pi / \kappa}$ is the Weierstrass function with periods $L$ and $i \pi / \kappa$. At $\kappa \rightarrow \infty$ this model gives back the short-range Heisenberg model. Another limiting case of this model is the $\kappa \rightarrow$ 0 limit, which yields the Haldane-Shastry model [75, 76], and which was widely studied in connection with exclusion statistics. Another possibility to define long-range deformations is to define the model through a series expansion in the deformation parameter. This was done for example for the dilatation operator of $\mathcal{N}=4$ SYM theory [77], which corresponds to an (asymptotically) integrable spin chain Hamiltonian. Integrability can then be defined perturbatively; for example if the deformed conserved charges are given by an expansion in the deformation parameter $g$ of the form ${ }^{13}$

$$
\mathrm{Q}_{r}(g)=\sum_{k \geq 0} \mathrm{Q}_{r}^{[k]} g^{2 k}
$$

then the terms in the expansion can be computed order by order and to test integrability to order $\ell$, one checks that

$$
\left[\mathrm{Q}_{r}(g), \mathrm{Q}_{s}(g)\right]=\mathcal{O}\left(g^{2(\ell+1)}\right) .
$$

We say then that the model is integrable up to $\ell$-loop order.

The BDS spin chain. An important example of a long-range spin chain that we will use in this work is the BDS chain [58]. It was defined in the perturbative sense as a longrange spin chain whose first three orders coincide with the dilatation operator of $\mathcal{N}=4$ supersymmetric Yang-Mills theory in the $\mathfrak{s u}(2)$ sector:

$$
D(g)=L+2 \sum_{k \geq 1} g^{2 k} D^{[k-1]}
$$

The coupling constant $g$ is related to the 't Hooft coupling constant of the gauge theory as $\lambda=16 \pi^{2} g^{2}$. The first three non-trivial orders of the dilatation operator were computed

\footnotetext{
${ }^{13}$ Here we suppose that only even powers of $g$ appear in the small $g$ expansion, as it is the case for the $\mathcal{N}=4$ SYM dilatation operator in the $\mathfrak{s u}(2)$ sector.
} 
by Beisert, Kristjansen and Staudacher [77] and they are given by

$$
\begin{aligned}
& D^{[0]}=\sum_{k=1}^{L}\left(1-\mathrm{P}_{k, k+1}\right), \\
& D^{[1]}=\sum_{k=1}^{L}\left(4 \mathrm{P}_{k, k+1}-\mathrm{P}_{k, k+2}-3\right), \\
& D^{[2]}=\sum_{k=1}^{L}\left(-14 \mathrm{P}_{k, k+1}+4 \mathrm{P}_{k, k+2}+10-\mathrm{P}_{k, k+3} \mathrm{P}_{k+1, k+2}+\mathrm{P}_{k, k+2} \mathrm{P}_{k+1, k+3}\right) .
\end{aligned}
$$

In the initial BDS paper [58], the model was defined beyond three-loop order by the Bethe ansatz equations it was supposed to obey:

$$
\left(\frac{x\left(u_{j}+\frac{i}{2}\right)}{x\left(u_{j}-\frac{i}{2}\right)}\right)^{L}=\prod_{\substack{k=1 \\ k \neq j}}^{M} \frac{u_{j}-u_{k}+i}{u_{j}-u_{k}-i}, \quad \quad e^{i p}=\frac{x\left(u+\frac{i}{2}\right)}{x\left(u-\frac{i}{2}\right)}
$$

with the rapidity map $x(u)$ and its inverse given by the Zhukovsky relation

$$
x(u)=\frac{u}{2}\left(1+\sqrt{1-\frac{4 g^{2}}{u^{2}}}\right), \quad u(x)=x+\frac{g^{2}}{x} .
$$

In [59] it was shown that the Hamiltonian (3.4) and the Bethe ansatz (3.6) can be obtained by reducing the one-dimensional half-filled Hubbard model to the spin sector. In principle, the higher order terms in (3.4) can be computed from perturbation theory of the Hubbard model, and at increasing perturbative order they involve interactions connecting more and more spins. The difference between the Hubbard model prediction and the Bethe ansatz equations appears at order $g^{2 L}$, which is the order at which wrapping interactions start to contribute.

Notably, the above Bethe equations for the BDS model equal the inhomogeneous Bethe equations (2.8) up to wrapping, if the inhomogeneities are fixed to [58 ${ }^{14}$

$$
\theta_{k}^{\mathrm{BDS}}=2 g \sin \frac{2 \pi k}{L}
$$

In consequence, the spectra of the two models are the same up to wrapping order and their Hamiltonians can be related by a similarity transformation [64]. In the subsequent sections we will pursue the investigation of this relation between the two spin chain models.

Beyond three loops neither the Hubbard model nor the inhomogeneous or BDS Hamiltonian yield the correct asymptotic dilatation operator of $\mathcal{N}=4$ super Yang-Mills theory. The different physical quantities obtained from these models have to be corrected by the so-called dressing phase contributions [78-80].

\footnotetext{
${ }^{14}$ For odd values of the length $L$ one should add a twist to the inhomogeneities that we neglect here for simplicity [59].
} 


\subsection{Boost operators}

In this section we review a general method for the construction of long-range spin chains using a deformation equation that preserves integrability $[63,64]$. We then discuss the BDS spin chain in this context.

The starting point for these long-range deformations is a given short-range system with mutually commuting Hamiltonians $\mathrm{Q}_{r}^{\mathrm{SR}}, r=2,3, \ldots$, (e.g. generated through (2.29)) that act locally and homogeneously on a spin chain. The long-range charges $\mathrm{Q}_{r}(g)$ are then defined by the deformation equation

$$
\frac{d}{d g} \mathrm{Q}_{r}(g)=i\left[\mathrm{X}(g), \mathrm{Q}_{r}(g)\right], \quad \mathrm{Q}_{r}(0) \equiv \mathrm{Q}_{r}^{[0]}=\mathrm{Q}_{r}^{\mathrm{SR}},
$$

whose solutions $\mathrm{Q}_{r}(g)$ are mutually commuting by construction. The generators of longrange deformations $\mathrm{X}(g)$ are constrained by the requirement that the $\mathrm{Q}_{r}(g)$ are local and homogeneous operators. In $[63,64]$ two main classes of generators $\mathrm{X}$ were identified and their physical interpretation was studied: ${ }^{15}$

$$
\begin{array}{lll}
\text { Boost charges: } & \mathrm{X}=\mathcal{B}\left[\mathrm{Q}_{r}\right]=\left[\mathrm{I} \mid \mathrm{Q}_{r}\right] & \text { (rapidity map) } \\
\text { Bilocal charges: } & \mathrm{X}=\left[\mathrm{Q}_{r} \mid \mathrm{Q}_{s}\right] & \text { (dressing phase) }
\end{array}
$$

Here the bilocal composition of two local operators $\mathcal{L}_{1}$ and $\mathcal{L}_{2}$ is defined as

$$
\left[\mathcal{L}_{1} \mid \mathcal{L}_{2}\right]=\sum_{k<\ell} \mathcal{L}_{1, k} \mathcal{L}_{2, \ell}
$$

Furthermore one may deform the charges by local operators $\mathrm{X}=\mathcal{L}$ which amounts to a similarity transformation not changing the spectrum; deformations with local conserved charges $\mathrm{X}=\mathrm{Q}_{r}$ are trivial. As mentioned before, the basis of local charges can be transformed without spoiling integrability. Typically the initial basis of short-range Hamiltonians is chosen in such a way that the charge $\mathrm{Q}_{r}(0)$ acts on at most $r$ neighboring spin chain sites at the same time.

Let us note that the boost operator (2.28) transforms under translations as

$$
\mathrm{U}_{0} \mathcal{B}[\mathcal{L}] \mathrm{U}_{0}^{-1}=\mathcal{B}[\mathcal{L}]+\mathcal{L},
$$

and is therefore not well-defined globally, since it is not compatible with the periodicity condition $\mathrm{U}_{0}^{L}=1$. However, if $\mathcal{L}$ is a conserved charge, the above boost recursions (2.29), (3.9) are well-defined locally, since the defining relations yield a local homogeneous operator. The fact that the boost is not well-defined globally insures that the deformation (3.9) is not just a similarity transformation and that the spectrum of the deformed model is different from the spectrum of the undeformed model. Similar arguments apply to deformations with bilocal charges.

The BDS spin chain introduced in the previous sections is obtained from a specific combination of the above boost deformations and basis transformations. Therefore we will

\footnotetext{
${ }^{15}$ Note that more types of generators can be specified depending on the deformed short-range model (see for instance the discussions of open boundary conditions [81] or the XXZ model [82]).
} 
here focus on boost deformations (3.10) of the XXX chain and leave the study of bilocal deformations in this context for future work. In order to obtain the full integrable model describing the asymptotic $\mathfrak{s u}(2)$ sector of $\mathcal{N}=4$ SYM theory (including the dressing phase contributions), also the bilocal charges (3.11) have to be switched on. The BDS and the full $\mathcal{N}=4$ SYM theory chain in the $\mathfrak{s u}(2)$ sector correspond to a specific choice of parameters in the large class of different long-range models that can be generated by the above method.

It is important to note that generically the interaction range of the solutions $\mathrm{Q}_{r}(g)$ to (3.9) increases with each order of the coupling parameter $g$. This implies that for a given spin chain, the range of the charge $\mathrm{Q}_{r}(g)$ exceeds the length of the chain from a given perturbative order in $g$. It is not known how to define the action of the charges beyond this so-called wrapping order. Hence, the validity of the considered long-range model is limited to the asymptotic regime of long states.

For our purposes in the following sections it is useful to distinguish two sets of commuting charges defined as deformations of the same short-range spin chain model (below we will consider deformations of the homogenoeus XXX spin chain). The charges $\mathrm{Q}_{r}(g)$ and $\overline{\mathrm{Q}}_{r}(g)$ are defined by the following two deformation equations: ${ }^{16}$

$$
\begin{aligned}
\frac{d}{d g} \mathrm{Q}_{r}(g) & =\sum_{k=3}^{\infty} \tau_{k} i\left[\mathcal{B}\left[\overline{\mathrm{Q}}_{k}(g)\right], \mathrm{Q}_{r}(g)\right], \\
\frac{d}{d g} \overline{\mathrm{Q}}_{r}(g) & =\sum_{k=3}^{\infty} \tau_{k}\left(i\left[\mathcal{B}\left[\overline{\mathrm{Q}}_{k}(g)\right], \overline{\mathrm{Q}}_{r}(g)\right]+\frac{r+k-2}{k-1} \overline{\mathrm{Q}}_{r+k-1}\right) .
\end{aligned}
$$

Furthermore we set $\mathrm{Q}_{r}(0)=\overline{\mathrm{Q}}_{r}(0)$ such that the two sets of charges differ only by the perturbative basis transformation on the r.h.s. of (3.15). As indicated above, the charges from the two sets commute among themselves by construction. In addition, the charges from different sets commute among each other since we have

$$
\frac{d}{d g}\left[\mathrm{Q}_{r}(g), \overline{\mathrm{Q}}_{s}(g)\right]=\sum_{k=3}^{\infty} \tau_{k}\left(i\left[\mathcal{B}\left[\overline{\mathrm{Q}}_{k}(g)\right],\left[\mathrm{Q}_{r}(g), \overline{\mathrm{Q}}_{s}(g)\right]\right]+\frac{r+k-2}{k-1}\left[\mathrm{Q}_{r}(g), \overline{\mathrm{Q}}_{s+k-1}(g)\right]\right) .
$$

Thus, if the charges commute at order zero (e.g. they are deformations of the same shortrange model), they also commute at higher orders in $g$. We conclude that both sets of charges should have the same basis of eigenstates. ${ }^{17}$ Following the lines of [64], one finds that both sets of charges are diagonalized by the same Bethe ansatz equations:

$$
\left(\frac{f\left(u+\frac{i}{2}\right)}{f\left(u-\frac{i}{2}\right)}\right)^{L}=\prod_{\substack{i=1 \\ i \neq k}}^{M} \frac{u_{j}-u_{k}+i}{u_{j}-u_{k}-i}, \quad e^{i p}=\frac{f\left(u+\frac{i}{2}\right)}{f\left(u-\frac{i}{2}\right)},
$$

\footnotetext{
${ }^{16}$ For a more detailed discussion of the relation between long-range deformations and the Bethe ansatz see $[63,64]$. For comparison to the notation used in [64] we note that $\tau_{k}=\Pi_{k} / d g$, where $\Pi_{k}$ is a one-form defined in that paper.

${ }^{17}$ Below we will fix $\tau_{k}$ such that the charges $\overline{\mathrm{Q}}_{r}(g)$ correspond to the BDS charges with minimized interaction range at each order in $g$. Ultimately we will be interested in comparing the eigenstates of the BDS charges to the eigenstates of the inhomogeneous model; for this purpose the basis of charges $\mathrm{Q}_{r}(g)$ is more convenient.
} 
where the rapidity map $f(u)$ is related to $\tau_{k}$ by

$$
\frac{d f(u)}{d g}=-\sum_{k=3}^{\infty} \frac{\tau_{k}}{(k-1)} \frac{1}{f^{k-2}} .
$$

When expressed as functions of the rapidity $u$, the one-magnon eigenvalues of the charges $\mathrm{Q}_{r}$ take the ordinary short-range form (2.22) and the deformation enters only via the Bethe equations. For the charges $\bar{Q}_{r}$, the one-magnon eigenvalues are given by

$$
\bar{q}_{r}(u)=\frac{i}{r-1}\left(\frac{1}{f\left(u+\frac{i}{2}\right)^{r-1}}-\frac{1}{f\left(u-\frac{i}{2}\right)^{r-1}}\right) .
$$

The eigenvalue functions turn out to be related by the following relation [82]:

$$
\bar{q}_{r}(u)=q_{r}(u)+\sum_{s=r+1}^{\infty} \gamma_{r, s}(g) q_{s}(u), \quad \frac{1}{f(u)^{r-1}}=\sum_{s=r}^{\infty} \gamma_{r, s}(g) \frac{r-1}{s-1} \frac{1}{u^{s-1}},
$$

where $\gamma_{r, s}(g)$ is defined by expansion of the second equation. Similarly, the corresponding charge operators should be related to each other.

Let us now compare the Bethe Ansatz equations (3.17) for the deformed spin chain with those for the inhomogeneous spin chain (2.8). We notice that they look similar, up to terms of order $\theta^{L}$ at least, if we write

$$
\frac{d \ln f(u)}{d u}=\frac{1}{L} \sum_{k=0}^{\infty} \frac{\sigma_{k}}{u^{k+1}}, \quad \text { with } \quad \sigma_{k}=\sum_{i=1}^{L} \theta_{i}^{k},
$$

and we relate $\tau_{k}$ to the symmetric sums $\sigma_{k}$ as prescribed by the relations (3.18) and (3.21). Since the functional form of the charge eigenvalues $q_{r}(u)$ also coincides with (2.22), we conclude that the spectra of the inhomogeneous model and the corresponding deformed model are the same. Because the spectrum depends only on the value of the symmetric sums, any permutation of the values of the impurities gives a model with the same spectrum (but not the same Hamiltonian). One may therefore suspect that the two types of models are mutually related by a unitary transformation, cf. [64]. In the next sections, this transformation is defined, and determined explicitly for the first two orders in perturbation. The values of the symmetric sums $\sigma_{k}$ in (3.21) can be translated into values of the coupling constants $\tau_{k}$ for the long-range deformations. These coupling constants define a whole family of long-range integrable models, since the values of the first $L$ symmetric sums can be tuned independently. Among these models we are particularly interested in the BDS model.

The BDS spin chain. Let us consider the recursive definition of the BDS chain in some more detail. In this case the rapidity map and its inverse are given by $f^{\mathrm{BDS}}(u) \equiv x(u)$, see (3.7). We may use this explicit form to evaluate (3.18) according to

$$
-\frac{d x(u)}{d g}=\frac{d u(x)}{d g} / \frac{d u(x)}{d x}=\frac{2 g x}{x^{2}-g^{2}}=\sum_{k=3}^{\infty} \frac{\tau_{k}^{\mathrm{BDS}}}{(k-1) x^{k-2}},
$$


such that writing the left hand side as a series we find the BDS expressions

$$
\tau_{2 k}^{\mathrm{BDS}}=0, \quad \tau_{2 k+1}^{\mathrm{BDS}}=4 k g^{2 k-1} .
$$

Then the BDS charges are given by $\overline{\mathrm{Q}}_{r}(g)$ as defined by $(3.15)$, where the $\overline{\mathrm{Q}}_{r}(0) \equiv \overline{\mathrm{Q}}_{r}^{[0]}$ denote the integrable charges of the XXX Heisenberg spin chain. In appendix B we give explicit solutions for the BDS charges in terms of boost deformations up to four-loop order.

In the following we will be interested in studying the effect of the above deformation on eigenstates of the charge operators and compare them to the inhomogeneous spin chain model. For this purpose it is more convenient to consider the charges $\mathrm{Q}_{r}(g)$. As indicated above, these charges have the same eigenstates as the BDS Hamiltonians $\overline{\mathrm{Q}}_{r}(g)$, and they have the same eigenvalues as those of the inhomogeneous models with the values of the symmetric sums given by ${ }^{18}$

$$
\sigma_{2 k+1}^{\mathrm{BDS}}=0, \quad \sigma_{2 k}^{\mathrm{BDS}}=L g^{2 k} \frac{(2 k) !}{(k !)^{2}} .
$$

In particular this is true for the model with the inhomogeneities specified in formula (3.8).

We thus consider the deformation equation (3.14) evaluated for the BDS connection (3.23). We may solve this equation in the form

$$
\mathrm{Q}_{r}(g)=e^{i \Phi(g)} \mathrm{Q}_{r}^{[0]} e^{-i \Phi(g)} \equiv \mathrm{S}_{\mathcal{B}} \mathrm{Q}_{r}^{[0]} \mathrm{S}_{\mathcal{B}}^{-1},
$$

where $\mathrm{Q}_{r}^{[0]}=\mathrm{Q}_{r}^{\mathrm{SR}}$ is the nearest-neighbor charge, and at the first perturbative orders we have

$$
\Phi(g)=g^{2} \mathrm{Y}^{[0]}+\frac{g^{4}}{2} \mathrm{Y}^{[1]}+\frac{g^{3}}{6}\left(\mathrm{Y}^{[2]}+\frac{i}{2}\left[\mathrm{Y}^{[1]}, \mathrm{Y}^{[0]}\right]\right)+\mathcal{O}\left(g^{8}\right)
$$

with

$$
\mathrm{Y}^{[\ell]}=2 \sum_{k=1}^{\ell+1} k \mathcal{B}\left[\overline{\mathrm{Q}}_{2 k+1}^{[\ell-k+1]}\right] .
$$

This explicitly defines the unitary boost transformation $\mathrm{S}_{\mathcal{B}}=\exp \left[i \Phi\left(g^{2}\right)\right]$ up to terms corresponding to the four-loop order in $\mathcal{N}=4$ SYM theory and can easily be written down to higher loops. Note that the local density $\Phi_{k}(g)$ of the operator $\Phi(g)$ is not periodic in the spin chain site $k$ since it is defined in terms of boost charges.

\section{Map from long-range to inhomogeneous models}

In this section we elaborate on the relation between long-range and inhomogeneous spin chains. After studying the unitary transformation $\mathrm{S}$ that relates the charge operators of the two models at leading orders, we argue that the operator S originates from a combination of boost operators and an inhomogeneous version of Baxter's corner transfer matrix discussed above. Finally we comment on the morphism defined by the S-operator and the relation to the theta-morphism introduced in [39].

\footnotetext{
${ }^{18}$ Let us emphasize that two inhomogeneous models obtained from one another by permutation of inhomogeneities have the same spectrum but different conserved charges, so they can be considered as being different.
} 


\subsection{S-operator}

We would like to understand better how the inhomogeneous spin chain model is related to the long-range system. To this end we follow the observation in [64] that the inhomogeneous charge operators can be mapped to the BDS charges by a unitary similarity transformation S. In [64] this transformation was specified for the BDS model and up to terms of order $g^{2}$. Here we study the relation between the inhomogeneous charges $\mathrm{Q}_{r}^{\theta}$ and generic long-range charges $\mathrm{Q}_{r}(g)$ obtained by the method of boost deformation: ${ }^{19}$

$$
\mathrm{Q}_{r}(g)=\mathrm{SQ}_{r}^{\theta} \mathrm{S}^{-1}
$$

The existence of the operator $\mathrm{S}$ is motivated by comparison of the spectra of the BDS chain and the inhomogeneous model for the BDS-values of the inhomogeneities [58].

Definition of the S-operator. In order to define the operator S, it is simplest to evaluate the same transformation as for the local charges on the shift operator

$$
\mathrm{U}(g)=\mathrm{S} \mathrm{U}_{\theta} \mathrm{S}^{-1},
$$

and to extract the form of $\mathrm{S}$ from this equation. Since both shift operators $\mathrm{U}(g)$ and $\mathrm{U}_{\theta}$ are defined to all orders, this yields an all-order definition of the S-operator in the parameter $g$. On the one hand, the inhomogeneous shift operator is defined by equation (2.18) from which we can read off its expansion $(\theta \sim g)$ :

$$
\mathrm{U}_{\theta}=\mathrm{U}_{0}\left[1-i \sum_{k=1}^{L} \theta_{k} \mathrm{H}_{k}-\frac{1}{2} \sum_{k, l=1}^{L} \theta_{k} \theta_{l} \mathrm{H}_{k} \mathrm{H}_{l}-\frac{1}{2} \sum_{k=1}^{L} \theta_{k-1} \theta_{k}[\mathrm{H}]_{k-1}\right]+\mathcal{O}\left(g^{3}\right) .
$$

For the boost induced long-range models on the other hand, we may apply the deformation equation (3.14) to the shift operator in analogy to deforming the local charges:

$$
\frac{d}{d g} \mathrm{U}(g)=i \sum_{k=3}^{\infty} \tau_{k}\left[\mathcal{B}\left[\overline{\mathrm{Q}}_{k}(g)\right], \mathrm{U}(g)\right], \quad \mathrm{U}(0)=\mathrm{U}_{0} .
$$

Here $\tau_{k}$ is defined by the rapidity map $f(u)$, which in turn is defined by the spectrum of the shift operator of the underlying model, cf. (3.17), (3.18) and [63, 64].

Let us assume that the expansion of $\tau_{2 k+1}$ starts at $g^{2 k-1}$ and that $\tau_{2 k}=0 .{ }^{20}$ Then we can use the shift property $(3.13)$ of the boost charges $\left(\left[\mathcal{B}[\mathcal{L}], \mathrm{U}_{0}\right]=-\mathrm{U}_{0} \mathcal{L}\right)$ to write down the first two non-trivial orders of $\mathrm{U}\left(g^{2}\right)$ :

$$
\mathrm{U}\left(g^{2}\right)=\mathrm{U}_{0}\left[1-i \bar{\tau}_{3} \mathrm{Q}_{3}^{\mathrm{SR}}+\bar{\tau}_{3}^{2}\left(\left[\mathcal{B}\left[\mathrm{Q}_{3}^{\mathrm{SR}}\right], \mathrm{Q}_{3}^{\mathrm{SR}}\right]-\frac{1}{2}\left(\mathrm{Q}_{3}^{\mathrm{SR}}\right)^{2}\right)-i\left(\bar{\tau}_{5}+\bar{\tau}_{3}^{2}\right) \mathrm{Q}_{5}^{\mathrm{SR}}\right]+\mathcal{O}\left(g^{6}\right) .
$$

\footnotetext{
${ }^{19}$ Note that the $\mathrm{Q}_{r}$ differ from the charges with minimized interaction range (e.g. the BDS charges) by a basis transformation as explained above. This does not make any difference for the transformation of eigenstates.

${ }^{20}$ The former assumption is motivated by the interaction range of the local charges being constrained by the gauge theory. The latter assumption corresponds to a parity conserving model. Both assumptions are satisfied for the BDS chain.
} 
Here we have defined ${ }^{21}$

$$
\bar{\tau}_{k}=\int_{0}^{g} \tau_{k}\left(g^{\prime}\right) d g^{\prime}
$$

Thus, both shift operators $\mathrm{U}_{\theta}$ and $\mathrm{U}(g)$ are defined to all orders in $g$ and (4.2) furnishes an all-order definition of the operator S. In the following section 2.1 we will elaborate more on the generic structure of the S-operator.

Let us now explicitly derive the perturbative expression for the unitary transformation that relates the two models up to order $g^{2}$. We make the same ansatz as in [64], namely

$$
\mathrm{S}=\exp i \sum_{k}\left[\nu_{k} \mathrm{H}_{k}+\frac{i}{2} \rho_{k}[\mathrm{H}]_{k-1}+\mathcal{O}\left(g^{3}\right)\right],
$$

but do not fix the constants $\nu_{k}$ and $\rho_{k}$ to their BDS values. Instead we will obtain generic expressions for $\nu_{k}$ and $\rho_{k}$ in terms of the periodic inhomogeneities $\theta_{k}$ that obey certain constraints. Here we assume that $\theta_{k} \sim g, \nu_{k} \sim g$ and $\rho_{k} \sim g^{2}$. We can now compare the two shift operators (4.3) and (4.5) and derive the constraints following from (4.2).

First order. We apply the ansatz (4.7) for the S-operator to the inhomogeneous shift and evaluate the expression at order $g$ :

$$
\mathrm{S} \mathrm{U}_{\theta} \mathrm{S}^{-1}=\mathrm{U}_{0}\left[1-i \sum_{k=1}^{L} \mathrm{H}_{k}\left(\nu_{k}-\nu_{k-1}+\theta_{k}\right)+\left(\nu_{L}-\nu_{0}\right) \mathrm{H}_{1}\right]+\mathcal{O}\left(g^{2}\right) .
$$

Here we have used that $\mathrm{H}_{k} \mathrm{U}_{0}=\mathrm{U}_{0} \mathrm{H}_{k+1}$. Since the long-range shift operator has no contribution at order $g^{1},(4.2)$ yields the constraints

$$
\nu_{k}-\nu_{k-1}+\theta_{k}=0, \quad \nu_{L}-\nu_{0}=0 .
$$

These equations are solved by the explicit expression

$$
\nu_{k}=\nu_{0}-\sum_{x=1}^{k} \theta_{x}
$$

and the periodicity condition for the first-order parameters yields

$$
\nu_{k+L}=\nu_{k} \quad \Rightarrow \quad \sum_{x=1}^{L} \theta_{x}=0 .
$$

The latter condition guarantees that the operator $\mathrm{S}$ is periodic, i.e. it represents a welldefined transformation on a periodic spin chain at the considered order.

Second order. Proceeding to terms at order $g^{2}$ in (4.2) we assume that the above constraints (4.9) hold. Also at this order we require $\mathrm{S}$ to be periodic which amounts to $\rho_{k+L}=\rho_{k}$. We may again evaluate the right hand side of (4.2) and after some manipulations (cf. appendix C) we arrive at

$$
\mathrm{S} \mathrm{U}_{\theta} \mathrm{S}^{-1}=\mathrm{U}_{0}\left[1+\frac{1}{2} \sum_{k=1}^{L}\left(\rho_{k}-\rho_{k-1}+\nu_{k-1}\left(\theta_{k}-\theta_{k-1}\right)\right)[\mathrm{H}]_{k-1}\right]+\mathcal{O}\left(g^{3}\right) .
$$

\footnotetext{
${ }^{21}$ For instance we have $\bar{\tau}_{2 k+1}^{\mathrm{BDS}}=2 g^{2 k}$.
} 
We may now compare this expression to the long-range shift operator (4.5) which gives the constraint equation for the second order parameters $\rho_{k}$ :

$$
\rho_{k}-\rho_{k-1}=\bar{\tau}_{3}-\nu_{k-1}\left(\theta_{k}-\theta_{k-1}\right)
$$

This equation is solved by (here we assume for simplicity $\nu_{0}=0$ )

$$
\rho_{k}=\rho_{0}+\bar{\tau}_{3} k-\sum_{x=1}^{k} \nu_{x-1}\left(\theta_{x}-\theta_{x-1}\right)=\rho_{0}+\bar{\tau}_{3} k-\theta_{k} \nu_{k}-\sum_{x=1}^{k} \theta_{x}^{2},
$$

and periodicity for the second order parameter yields

$$
\rho_{k+L}=\rho_{k} \quad \Rightarrow \quad \sum_{x=1}^{L} \theta_{x}^{2}=\sigma_{2}=\bar{\tau}_{3} L .
$$

Conclusion. In this section we have given an all-order definition of the operator $\mathrm{S}$ by the transformation relating the inhomogeneous and long-range shift operators (4.2). We have then computed the operator S up to terms of order $g^{2}$. The S-operator takes the form

$$
\mathrm{S}=\exp i\left[\bar{\tau}_{3} \mathcal{B}\left[\mathrm{Q}_{3}^{\mathrm{SR}}\right]+\sum_{k=1}^{L}\left(\nu_{k} \mathrm{H}_{k}+\frac{i}{2} \hat{\rho}_{k}[\mathrm{H}]_{k-1}\right)\right]+\mathcal{O}\left(g^{3}\right)
$$

and for the inhomogeneities set to the BDS values it gives the expression already determined in [64]. Notably the S-operator can be split into two contributions

$$
\mathrm{S}=\mathrm{S}_{\mathcal{B}} \times \mathrm{S}_{\theta}^{-1}, \quad \mathrm{~S}_{\mathcal{B}}=\exp i \Phi, \quad \mathrm{S}_{\theta}^{-1}=\exp i \Theta .
$$

Here the boost and inhomogeneous generator are given by

$$
\Phi=\bar{\tau}_{3} \mathcal{B}\left[\mathrm{Q}_{3}^{\mathrm{SR}}\right]+\mathcal{O}\left(g^{4}\right), \quad \Theta=\sum_{k=1}^{L}\left(\nu_{k} \mathrm{H}_{k}+\frac{i}{2} \hat{\rho}_{k}[\mathrm{H}]_{k-1}\right)+\mathcal{O}\left(g^{3}\right) .
$$

and we have defined the inhomogeneous parameter $\hat{\rho}_{k}$ to separate the boost and inhomogeneous piece:

$$
\hat{\rho}_{k}=\rho_{0}-\theta_{k} \nu_{k}-\sum_{x=1}^{k} \theta_{x}^{2} .
$$

Note that the boost part agrees with the first order of (3.26) as expected. This splitting into a boost and an inhomogeneous piece is natural knowing that the boost deformations generate the long-range model from the short-range (here Heisenberg) model (cf. section 3.1). In particular this implies two important features for the inhomogeneous part $S_{\theta}^{-1}$ of the S-operator:

- In the bulk, $\mathrm{S}_{\theta}^{-1}$ sets all inhomogeneities to zero and hence represents the generator of the inhomogeneous rapidity shift as indicated in section 2.1 .

- At the boundary, $\mathrm{S}_{\theta}^{-1}$ completes $\mathrm{S}_{\mathcal{B}}$ to a periodic operator $\mathrm{S}$. 
Remarkably, the expression for $\mathrm{S}_{\theta}^{-1}$ in (4.17) agrees with the expansion of the inhomogeneous corner transfer matrix $\mathcal{A}_{\theta}(2.35)$. That is to say that the parameters $\nu_{k}$ and $\hat{\rho}_{k}$ are the same functions of $\theta$ as defined in (2.36) (for $\nu_{0}=0$ and $\rho_{0}=0$ ). We have thus found that the expansion of $\mathrm{S}_{\theta}^{-1}$ is identical with the expansion of the inhomogeneous CTM at first orders:

$$
\mathrm{S}_{\theta}^{-1}=\mathcal{A}_{\theta}(0)+\mathcal{O}\left(\theta^{3}\right) .
$$

Assuming that the map between $\mathrm{S}_{\theta}$ and $\mathcal{A}_{\theta}$ holds at higher orders, it seems natural to use the CTM to define the operator $\mathrm{S}_{\theta}$. In fact, the inhomogeneous CTM is defined to all orders in $\theta$ according to (2.34) in terms of R-matrices. Together with the boost deformations discussed in the previous sections this could furnish an explicit definition of the complete $\mathrm{S}$-operator. Note that at higher orders it remains to be shown that an operator $\mathrm{S}_{\theta}$ defined in this way has the desired property to combine with the boost part into the transformation translating between long-range and inhomogeneous spin chains.

\subsection{Morphism property and scalar products}

In the previous section we have shown how to obtain the integrals of motion for the longrange (LR) model by transforming the inhomogeneous integrals of motion with the unitary operator S. The same unitary transformation can be applied to the monodromy matrix $\mathrm{M}$ as well,

$$
\mathrm{M}^{\mathrm{LR}}(u)=\mathrm{SM}(u ; \boldsymbol{\theta}) \mathrm{S}^{-1}
$$

where the values of $\boldsymbol{\theta}$ are chosen as explained in (3.21). It is straightforward to show that the monodromy matrix of the long-range model $\mathrm{M}^{\mathrm{LR}}(u)$ obeys the Yang-Baxter equation, and that its matrix elements obey the same algebra as the inhomogeneous (or homogeneous) ones (2.6). The unitary transformation is therefore a morphism of the Yangian algebra,

$$
\mathrm{M}_{\alpha}^{\mathrm{LR}}(u) \mathrm{M}_{\beta}^{\mathrm{LR}}(v)=\mathrm{S} \mathrm{M}_{\alpha}(u ; \boldsymbol{\theta}) \mathrm{M}_{\beta}(v ; \boldsymbol{\theta}) \mathrm{S}^{-1},
$$

for any spaces $\alpha$ and $\beta$. It is important to note that this morphism works for periodic chains of arbitrary length, up to wrapping order $g^{2 L}$. This is in contradistinction to the morphism considered in [38, 62], based on the Dunkl operators, where a defect was added at the point where the chain closes. Of course, the difference between the two is small for large chains. Let us explore the consequences of this morphism. First, the Bethe vectors for the long-range model, on-shell or of-shell, can be written simply as

$$
|\mathbf{u}\rangle_{\mathrm{LR}}=\mathrm{S}|\mathbf{u} ; \boldsymbol{\theta}\rangle, \quad \operatorname{LR}\langle\mathbf{u}|=\langle\mathbf{u} ; \boldsymbol{\theta}| \mathrm{S}^{-1} .
$$

This means that the scalar products, including the norms, are the same for the long-range model and the corresponding inhomogeneous model,

$$
{ }_{\mathrm{LR}}\langle\mathbf{v} \mid \mathbf{u}\rangle_{\mathrm{LR}}=\langle\mathbf{v} ; \boldsymbol{\theta} \mid \mathbf{u} ; \boldsymbol{\theta}\rangle
$$

The evaluation of the scalar products in the long-range model, up to wrapping order, is then straightforward. According to Slavnov [83], the scalar product of an on-shell and and 
off-shell vector can be written in terms of a determinant

$$
\langle\mathbf{v} ; \boldsymbol{\theta} \mid \mathbf{u} ; \boldsymbol{\theta}\rangle=\prod_{j=1}^{N} d\left(u_{j}\right) a\left(v_{j}\right) \mathscr{S}_{\mathbf{u}, \mathbf{v}}, \quad \mathscr{S}_{\mathbf{u}, \mathbf{v}}=\frac{\operatorname{det}_{j k} \Omega\left(u_{j}, v_{k}\right)}{\operatorname{det}_{j k} \frac{1}{u_{j}-v_{k}+i}} .
$$

The Slavnov kernel $\Omega(u, v)$ is ${ }^{22}$

$$
\Omega(u, v)=t(u-v)-e^{2 i p_{\mathbf{u}}(v)} t(v-u), \quad t(u)=\frac{1}{u}-\frac{1}{u+i},
$$

where $p_{\mathbf{u}}(u)$ is the quasimomentum defined modulo $\pi$ by

$$
e^{2 i p(u)}=\frac{Q_{\mathbf{u}}(u+i)}{Q_{\mathbf{u}}(u-i)} \frac{Q_{\boldsymbol{\theta}}(u-i / 2)}{Q_{\boldsymbol{\theta}}(u+i / 2)} .
$$

Here $Q_{\boldsymbol{\theta}}(u)=\prod_{j=1}^{L}\left(u-\theta_{j}\right)$ and $Q_{\mathbf{u}}(u)=\prod_{j=1}^{N}\left(u-u_{j}\right)$ are Baxter polynomials. Taking the limit $\mathbf{v} \rightarrow \mathbf{u}$, one obtains a determinant expression for the norm (the Gaudin-Korepin determinant)

$$
\begin{aligned}
\langle\mathbf{u} ; \boldsymbol{\theta} \mid \mathbf{u} ; \boldsymbol{\theta}\rangle & =\frac{\prod_{j=1}^{N} 2 d^{2}\left(u_{j}\right)}{\prod_{j<k}\left(u_{j}-u_{k}\right)^{2}} \operatorname{det}\left(\frac{\left(1-\delta_{j k}\right)}{u_{j k}^{2}+1}+\left.\partial_{u} p_{\mathbf{u}}(u)\right|_{u_{j}} \delta_{j k}\right) \\
& =\frac{\prod_{j=1}^{N} 2 d^{2}\left(u_{j}\right)}{\prod_{j<k}\left(u_{j}-u_{k}\right)^{2}} \operatorname{det}_{j k} \partial_{u_{j}} p_{\mathbf{u}}\left(u_{k}\right) .
\end{aligned}
$$

The diagonal term in the above determinant should be understood as $\left.\partial_{u} p_{\mathbf{u}}(u)\right|_{u=u_{j}}$.

In [84] the Slavnov determinant formula was simplified, based on the results in [26], to the symmetric-looking formula ${ }^{23}$

$$
\langle\mathbf{v} ; \boldsymbol{\theta} \mid \mathbf{u} ; \boldsymbol{\theta}\rangle=\prod_{j=1}^{N} d\left(u_{j}\right) a\left(v_{j}\right) \mathscr{A}_{\mathbf{u} \cup \mathbf{v}, \boldsymbol{\theta}}^{+}=\prod_{j=1}^{N} d\left(u_{j}\right) d\left(v_{j}\right) \mathscr{A}_{\mathbf{u} \cup \mathbf{v}, \boldsymbol{\theta}}^{-}
$$

where the functional $\mathscr{A}_{\mathbf{w}, \boldsymbol{\theta}}^{ \pm}$is defined as follows:

$$
\mathscr{A}_{\mathbf{w}, \boldsymbol{\theta}}^{ \pm} \equiv \frac{1}{\Psi_{\mathbf{w}, \boldsymbol{\theta} \pm i / 2}} \prod_{j=1}^{2 N}\left(1-e^{ \pm i \partial / \partial w_{j}}\right) \Psi_{\mathbf{w}, \boldsymbol{\theta} \pm i / 2}, \quad \Psi_{\mathbf{w}, \boldsymbol{\theta}}=\frac{\prod_{j<k}^{2 N}\left(w_{j}-w_{k}\right)^{2}}{\prod_{j=1}^{2 N} \prod_{l=1}^{L}\left(w_{j}-\theta_{l}\right)}
$$

The two representations are compatible due to the identity below, which follows directly from the definition,

$$
\mathscr{A}_{\mathbf{w}, \boldsymbol{\theta}}^{+}=\prod_{j=1}^{2 N} \frac{Q_{\boldsymbol{\theta}}^{-}\left(w_{j}\right)}{Q_{\boldsymbol{\theta}}^{+}\left(w_{j}\right)} \mathscr{A}_{\mathbf{w}, \boldsymbol{\theta}}^{-}, \quad \mathbf{w}=\mathbf{u} \cup \mathbf{v},
$$

\footnotetext{
${ }^{22}$ We are using a different normalization than in the original paper [83].

${ }^{23}$ In proving (4.29) it is essential that the rapidities $\mathbf{u}$ are on-shell. In [84] only the first identity is proved; the second one is obtained by the same method. With the chosen normalization of the monodromy matrix we should use the second representation.
} 
where we use the consequence of the Bethe equations,

$$
\prod_{j=1}^{N} \frac{Q_{\boldsymbol{\theta}}^{-}\left(u_{j}\right)}{Q_{\boldsymbol{\theta}}^{+}\left(u_{j}\right)} \equiv \prod_{j=1}^{N} \frac{d\left(u_{j}\right)}{a\left(u_{j}\right)}=1 .
$$

As a consequence, the functionals $\mathscr{A}^{+}$and $\mathscr{A}^{-}$differ by a phase factor, with the phase equal to the total momentum of the magnons with rapidities $\mathbf{v}$ of the off-shell state. Since the phase factor does not have physical meaning, sometimes we will omit the \pm . The $\mathscr{A}$-functional can be also written in the form of a Fredholm-like determinant [85]

$$
\mathscr{A}_{\mathbf{u}, \boldsymbol{\theta}}^{ \pm}=\operatorname{det}_{j k}\left(\delta_{j k}-\frac{i E_{j}^{ \pm}}{u_{j}-u_{k}+i}\right), \quad E_{j}^{ \pm} \equiv \frac{Q_{\boldsymbol{\theta}}\left(u_{j} \mp i / 2\right)}{Q_{\boldsymbol{\theta}}\left(u_{j} \pm i / 2\right)} \prod_{k(\neq j)} \frac{u_{j}-u_{k} \pm i}{u_{j}-u_{k}} .
$$

The inhomogeneous $\mathscr{A}$-functional is a symmetric function of the variables $\boldsymbol{\theta}$ and it can be straightforwardly transformed into a long-range $\mathscr{A}$-functional, $\mathscr{A}_{\mathbf{u}, \boldsymbol{\theta}}=\mathscr{A}_{\mathbf{u}}^{\mathrm{LR}}+\mathcal{O}\left(g^{2 L}\right)$, with

$$
\mathscr{A}_{\mathbf{u}}^{\mathrm{LR}, \pm}=\operatorname{det}_{j k}\left(\delta_{j k}-\frac{i E_{j}^{\mathrm{LR}, \pm}}{u_{j}-u_{k}+i}\right), \quad E_{j}^{\mathrm{LR}, \pm} \equiv\left(\frac{f\left(u_{j} \mp i / 2\right)}{f\left(u_{j} \pm i / 2\right)}\right)^{L} \prod_{k(\neq j)} \frac{u_{j}-u_{k} \pm i}{u_{j}-u_{k}}
$$

The function $f(u)$ was defined in (3.21) in terms of the symmetric sums $\sigma_{k}=\sum_{j} \theta_{j}^{k}$ and it enters the long-range Bethe ansatz (3.17). A formula equivalent to (4.34) is implicit in [38] and was subsequently conjectured and checked for the norms in [39]. Let us emphasize that we do not need to know the explicit form of the operator $\mathrm{S}$ in order to compute the scalar products of the long-range spin chain. The above formulas are readily adapted for going to the semiclassical limit where $L$ and $N$ are large.

The dressing phase and the inhomogeneities. The inhomogeneities can also be used to emulate the effect of the dressing phase, provided that we allow their value to depend on the value of the rapidities. This amounts to allowing the symmetric sums to be symmetric functions of the rapidities $\mathbf{u}\left(\sigma_{k}=\sigma_{k}(\mathbf{u})\right)$ so that we have for the eigenstates of the model with the dressing phase, for example with the BES phase [80],

$$
|\mathbf{u}\rangle_{\mathrm{BES}}=\mathrm{S}(\mathbf{u})|\mathbf{u} ; \boldsymbol{\theta}(\mathbf{u})\rangle .
$$

Since the operator $\mathrm{S}(\mathbf{u})$ depends now on the state on which it acts, we cannot compute the scalar products in the same straightforward manner, but at least we can compute the norms of the Bethe ansatz vectors,

$$
{ }_{\mathrm{BES}}\langle\mathbf{u} \mid \mathbf{u}\rangle_{\mathrm{BES}}=\langle\mathbf{u} ; \boldsymbol{\theta}(\mathbf{u}) \mid \mathbf{u} ; \boldsymbol{\theta}(\mathbf{u})\rangle=\lim _{\mathbf{v} \rightarrow \mathbf{u}}\langle\mathbf{v} ; \boldsymbol{\theta}(\mathbf{u}) \mid \mathbf{u} ; \boldsymbol{\theta}(\mathbf{u})\rangle
$$

The above scalar product can be computed as a usual scalar product in the inhomogeneous model. In particular, the last expression, before taking the limit, is a usual scalar product with one vector on-shell and the other off-shell, which can be computed using (4.25). After replacing the symmetric sums with their values $\sigma_{k}(\mathbf{u})$ we get the matrix (4.26), with $p_{\mathbf{u}}(v)$ replaced by $p_{\mathbf{u}}^{\mathrm{BES}}(v)$. After taking the limit $\mathbf{v} \rightarrow \mathbf{u}$, we obtain

$$
\operatorname{BES}\langle\mathbf{u} \mid \mathbf{u}\rangle_{\mathrm{BES}}=\frac{\prod_{j=1}^{N} 2 d^{2}\left(u_{j}\right)}{\prod_{j<k}\left(u_{j}-u_{k}\right)^{2}} \operatorname{det}\left(\frac{\left(1-\delta_{j k}\right)}{u_{j k}^{2}+1}+\left.\partial_{u} p_{\mathbf{u}}^{\mathrm{BES}}(u)\right|_{u_{j}} \delta_{j k}\right) .
$$


This formula looks different from the conjecture for the norm with the dressing phase given by Gromov and Vieira [39].

\subsection{Morphism and theta-morphism}

In this section we analyze the relation between the unitary S-transformation and the "thetamorphism" introduced by Gromov and Vieira [37, 39]. The idea of Gromov and Vieira was to construct the states of the BDS long-range model by acting with a differential operator, which they called theta-morphism, on the states of the inhomogeneous model. We find that a purely differential operator cannot realize the morphism property, see below. The failure to fulfill the morphism property results in the cross-terms of [39]. Instead, we introduce the morphism associated to the S-operator via

$$
\mathrm{M}^{\mathrm{BDS}}(u)=\left.\mathrm{S} \mathrm{M}\left(u ; \boldsymbol{\theta}^{\mathrm{BDS}}\right) \mathrm{S}^{-1} \equiv \mathscr{D}_{\theta} \mathrm{M}(u ; \boldsymbol{\theta})\right|_{\theta=0} .
$$

This definition has the following advantages:

- Unlike the Gromov-Vieira theta-morphism, the inhomogeneity translation plus the unitary transformation amounts to an exact morphism of the Yangian algebra. It works not only on Bethe vectors, but on arbitrary elements of the monodromy matrix.

- The S-transformation produces the required boundary terms and therefore it is free of the "cross-term" issue which complicates the computation in [39].

- The relation to boost deformations and the inhomogeneous corner transfer matrix indicates a natural extension to higher orders.

We find that, up to terms of order $\mathcal{O}\left(g^{3}\right)$, the action of $\mathscr{D}_{\theta}$ on (an arbitrary matrix element of) the monodromy matrix $\mathrm{M}$ amounts to

$$
\begin{aligned}
& \left.\mathscr{D}_{\theta} \mathrm{M}(u ; \boldsymbol{\theta})\right|_{\theta=0} \\
& \equiv \mathrm{M}-\frac{g^{2}}{2} \sum_{k=1}^{L} \mathrm{D}_{k}^{2} \mathrm{M}-g^{2} \sum_{k=1}^{L} \mathrm{D}_{k} \mathrm{M}\left(\mathrm{P}_{k, k+1}+\delta_{k, L} \mathrm{Q}_{2}\right)+\left.g^{2}\left[\frac{\mathrm{Q}_{2}^{2}}{2}+i \mathrm{Q}_{3}+\mathrm{P}_{1 L} \mathrm{Q}_{2}, \mathrm{M}\right]\right|_{\theta=0} .
\end{aligned}
$$

To avoid cumbersome notations, in this section and below, we drop the indices on $\mathrm{Q}_{r}^{[0]}=$ $\mathrm{Q}_{r}^{\mathrm{SR}}$ to denote the leading order charges simply by $\mathrm{Q}_{r}$, and we have ${ }^{24}$

$$
\mathrm{D}_{k} \equiv i\left(\partial_{k}-\partial_{k+1}\right)=i\left(\partial_{\theta_{k}}-\partial_{\theta_{k+1}}\right) .
$$

The operator $\mathscr{D}_{\theta}$ differs from the theta-morphism of Gromov and Vieira [39] given by

$$
\mathcal{D}_{\theta}^{\mathrm{GV}}=1-\frac{g^{2}}{2} \sum_{k=1}^{L} \mathrm{D}_{k}^{2}+\mathcal{O}\left(g^{4}\right),
$$

by the last two terms in the second line of equation (4.39). These two extra terms account for the cross-terms in [39] and they insure that the morphism property is exact

$$
\left.\mathscr{D}_{\theta}\left(\mathrm{M}\left(u_{1} ; \boldsymbol{\theta}\right) \mathrm{M}\left(u_{2} ; \boldsymbol{\theta}\right)\right)\right|_{\theta=0}=\left.\left.\mathscr{D}_{\theta} \mathrm{M}\left(u_{1} ; \boldsymbol{\theta}\right)\right|_{\theta=0} \mathscr{D}_{\theta} \mathrm{M}\left(u_{2} ; \boldsymbol{\theta}\right)\right|_{\theta=0} .
$$

\footnotetext{
${ }^{24}$ Our definition of $\mathrm{D}_{k}$ differs from that of [39] by a factor of $i$.
} 
On the Bethe vectors, the action of the operator $\mathscr{D}_{\theta}$ reduces to

$$
|\mathbf{u}\rangle_{\mathrm{BDS}}=\mathrm{S}\left|\mathbf{u} ; \boldsymbol{\theta}^{\mathrm{BDS}}\right\rangle=\left.\mathscr{D}_{\theta}|\mathbf{u} ; \boldsymbol{\theta}\rangle\right|_{\theta=0}=\left.\left(\mathcal{D}_{\theta}^{\mathrm{GV}}+\frac{g^{2}}{2}\left(\mathrm{Q}_{2}^{2}+2 i \mathrm{Q}_{3}+2 \mathrm{P}_{1 L} \mathrm{Q}_{2}\right)\right)|\mathbf{u} ; \boldsymbol{\theta}\rangle\right|_{\theta=0} .
$$

To obtain this expression, we use that $\sum_{k} \mathrm{D}_{k}=0$ and that the vacuum eigenvalues of $\mathrm{Q}_{2}$ and $\mathrm{Q}_{3}$ are zero. If the Bethe vectors are on-shell, the charges $\mathrm{Q}_{2}$ and $\mathrm{Q}_{3}$ become numbers and we obtain

$$
\begin{aligned}
|\mathbf{u}\rangle_{\mathrm{BDS}} & =\left.\left[\mathcal{D}_{\theta}^{\mathrm{GV}}+g^{2}\left(\frac{1}{2} E_{2}^{2}+E_{2}+i E_{3}-\mathrm{H}_{1 L} \mathrm{Q}_{2}\right)\right]|\mathbf{u} ; \boldsymbol{\theta}\rangle\right|_{\theta=0} \\
& =\left[1+g^{2}\left(\frac{1}{2} E_{2}^{2}+E_{2}+i E_{3}\right)\right]|\mathbf{u}\rangle_{\mathrm{BDS}}^{\mathrm{GV}} .
\end{aligned}
$$

We thus see that our eigenvectors differ from those of Gromov and Vieira by a statedependent factor. The imaginary contribution does not affect the norms, while the real part changes the normalization. The scalar products of two arbitrary Bethe states, on-shell or off-shell, is:

$$
{ }_{\mathrm{BDS}}\langle\mathbf{u} \mid \mathbf{v}\rangle_{\mathrm{BDS}}=\left.\mathscr{D}_{\theta}\langle\mathbf{u} ; \boldsymbol{\theta} \mid \mathbf{v} ; \boldsymbol{\theta}\rangle\right|_{\theta=0}=\left.\mathcal{D}_{\theta}^{\mathrm{GV}}\langle\mathbf{u} ; \boldsymbol{\theta} \mid \mathbf{v} ; \boldsymbol{\theta}\rangle\right|_{\theta=0}
$$

Let us now sketch the derivation of the expression (4.39). More details are given in appendix D. First, we account for the shift in the inhomogeneities by

$$
\mathrm{M}\left(u ; \boldsymbol{\theta}^{\mathrm{BDS}}\right)=\left.\exp \left(\sum_{l=1}^{L} \theta_{l}^{\mathrm{BDS}} \partial_{\theta_{l}}\right) \mathrm{M}(u ; \boldsymbol{\theta})\right|_{\theta=0} .
$$

The second ingredient is to transform the action of the permutation operators contained in $\mathrm{S}$ into derivatives. The simplest ones were given in [37],

$$
\left[\mathrm{H}_{k}, \mathrm{M}(u)\right]=\left.\left(\mathrm{Q}_{2} \delta_{k, L}-\mathrm{D}_{k}\right) \mathrm{M}(u ; \boldsymbol{\theta})\right|_{\theta=0} .
$$

At higher orders in the expansion we have to deal with multiple permutations. The case of non-overlapping permutations is simple,

$$
\left[\mathrm{H}_{l},\left[\mathrm{H}_{k}, \mathrm{M}\right]\right]=\left(\mathrm{Q}_{2} \delta_{l, L}-\mathrm{D}_{l}\right)\left(\mathrm{Q}_{2} \delta_{k, L}-\mathrm{D}_{k}\right) \mathrm{M}, \quad|l-k|>1 .
$$

For overlapping permutations in the bulk, $k \neq L-1, L$, we obtain,

$$
\begin{aligned}
{\left[[\mathrm{H}]_{k}, \mathrm{M}\right]=} & \frac{1}{2}\left(\mathrm{D}_{k}^{2}-\mathrm{D}_{k+1}^{2}\right) \mathrm{M}+\mathrm{D}_{k} \mathrm{MP}_{k, k+1}-\mathrm{D}_{k+1} \mathrm{MP}_{k+1, k+2} \\
{\left.\left[\{\mathrm{H}\}_{k}, \mathrm{M}\right]\right]=} & \frac{1}{2}\left(\mathrm{D}_{k}^{2}+\mathrm{D}_{k+1}^{2}\right) \mathrm{M}+\mathrm{D}_{k} \mathrm{MP}_{k, k+1}+\mathrm{D}_{k+1} \mathrm{MP}_{k+1, k+2} \\
& +2 \mathrm{D}_{k} \mathrm{D}_{k+1} \mathrm{M}+2 \mathrm{D}_{k} \mathrm{MP}_{k+1, k+2}+\mathrm{D}_{k+1} \mathrm{MP}_{k, k+1},
\end{aligned}
$$

where $[\mathrm{H}]_{k}=\left[\mathrm{H}_{k}, \mathrm{H}_{k+1}\right]$ and $\{\mathrm{H}\}_{k}=\left\{\mathrm{H}_{k}, \mathrm{H}_{k+1}\right\}$. When $k=L-1, L$ the action in (4.48) has to be supplemented with boundary terms,

$$
\begin{aligned}
{\left[[\mathrm{H}]_{L-1}, \mathrm{M}\right] } & =\left[[\mathrm{H}]_{L-1}, \mathrm{M}\right]_{\text {bulk }}+\left[\delta_{\text {bound }}-2 i \mathrm{Q}_{3}, \mathrm{M}\right] \\
{\left[[\mathrm{H}]_{L}, \mathrm{M}\right] } & =\left[[\mathrm{H}]_{L}, \mathrm{M}\right]_{\text {bulk }}-\left[\delta_{\text {bound }}, \mathrm{M}\right], \quad \delta_{\text {bound }}=\left(\frac{1}{2} \mathrm{Q}_{2}^{2}+i \mathrm{Q}_{3}+\mathrm{P}_{1 L} \mathrm{Q}_{2}\right)
\end{aligned}
$$


These expressions, together with the action of overlapping $\mathrm{D}_{k}$ and $\mathrm{H}_{l}$ that are derived in appendix D, are all we need to obtain (4.39), provided that we choose $\nu_{0}=\rho_{0}=0$. Let us notice that the expressions (4.48), (4.49), (4.50) obey the Leibniz rule, e.g.

$$
\left[[\mathrm{H}]_{k}, \mathrm{M}_{1} \mathrm{M}_{2}\right]=\mathrm{M}_{1}\left[[\mathrm{H}]_{k}, \mathrm{M}_{2}\right]+\left[[\mathrm{H}]_{k}, \mathrm{M}_{1}\right] \mathrm{M}_{2} .
$$

We can thus safely replace $\mathrm{M}$ by any product of monodromy matrices in all the commutators above. This feature is at the origin of the morphism property.

\section{$5 \quad$ Three-point function of $\mathfrak{s u}(2)$ fields beyond tree level}

We consider operators which have definite conformal dimensions $\Delta^{(1)}, \Delta^{(2)}$ and $\Delta^{(3)}$. The three-point function of three renormalized operators $\mathcal{O}^{(1)}, \mathcal{O}^{(2)}$ and $\mathcal{O}^{(3)}$ in the $\mathcal{N}=4$ gauge theory is almost entirely fixed by conformal symmetry,

$$
\left\langle\mathcal{O}^{(1)}\left(x_{1}\right) \mathcal{O}^{(2)}\left(x_{2}\right) \mathcal{O}^{(3)}\left(x_{3}\right)\right\rangle=\frac{N_{c}^{-1} \sqrt{L^{(1)} L^{(2)} L^{(3)}} C_{123}\left(g^{2}\right)}{\left|x_{12}\right|^{\Delta^{(1)}+\Delta^{(2)}-\Delta^{(3)}}\left|x_{13}\right|^{\Delta^{(1)}+\Delta^{(3)}-\Delta^{(2)}}\left|x_{23}\right|^{\Delta^{(2)}-\Delta^{(3)}-\Delta^{(1)}}} .
$$

The only part which remains to be evaluated is the scheme-independent structure constant

$$
C_{123}\left(g^{2}\right)=\sum_{k \geq 0} C_{123}^{[k]} g^{2 k}
$$

A particular embedding of $\mathfrak{s u}(2)$ fields in the $\mathfrak{s o}(4)$ sector. The structure constant depends on the quantum numbers of the three $\mathfrak{s u}(2)$ fields. Each $\mathfrak{s u}(2)$ type field is characterized by an on-shell Bethe state in the XXX chain, as well as by the embedding of the $\mathfrak{s u}(2)$ sector in $\mathfrak{s o}(6) .{ }^{25}$ The correlation function considered here, as well as in [23, 24, 27, 39], corresponds to a particular choice of the $\mathfrak{s u}(2)$ sectors to which the three operators belong. With this choice, the three $\mathfrak{s u}(2)$ operators are traces of two complex bosons

$$
\mathcal{O}^{(1)} \in\{Z, X\}, \quad \mathcal{O}^{(2)} \in\{\bar{Z}, \bar{X}\}, \quad \mathcal{O}^{(3)} \in\{Z, \bar{X}\},
$$

for example $\mathcal{O}^{(1)}\left(x_{1}\right) \sim \operatorname{Tr} Z Z X X X \ldots X X Z X\left(x_{1}\right)$. Chosen in this way, the three $\mathfrak{s u}(2)$ operators belong to the $\mathfrak{s o}(4) \sim \mathfrak{s u}(2)_{R} \oplus \mathfrak{s u}(2)_{L}$ subsector of $\mathfrak{s o}(6)$. The two $\mathfrak{s u}(2)$ groups act by left and right multiplication of the complex matrix

$$
\left(\begin{array}{cc}
\Phi_{1}+i \Phi_{2} & \Phi_{3}+i \Phi_{4} \\
-\Phi_{3}+i \Phi_{4} & \Phi_{1}-i \Phi_{2}
\end{array}\right)=\left(\begin{array}{cc}
Z & X \\
-\bar{X} & \bar{Z}
\end{array}\right)
$$

The operators $\mathcal{O}^{(1)}$ and $\mathcal{O}^{(2)}$ belong to two dual $\mathfrak{s u}(2)_{R}$ sectors, while the operator $\mathcal{O}^{(3)}$ belongs to the $\mathfrak{s u}(2)_{L}$ sector. Under the right multiplications, $\{Z, X\}$ and $\{-\bar{X}, \bar{Z}\}$ transform as $\mathfrak{s u}(2)$ doublets. Under the left multiplications, the pairs of fields $\{Z,-\bar{X}\}$ and $\{X, \bar{Z}\}$ transform as $\mathfrak{s u}(2)_{R}$ doublets [87].

\footnotetext{
${ }^{25}$ The choice of the $\mathfrak{s u}(2)$ sector is determined by a set of global coordinates (angles). One can argue that the dependence of the three-point function on the global angles factorizes [86], but we will not discuss this issue here.
} 
The spin-chain lengths and the magnon numbers of the three states are related to the two $\mathfrak{s o}(4)$ charges by

$$
\begin{array}{ll}
L^{(1)}=+J_{1}^{(1)}+J_{2}^{(1)}, & N^{(1)}=+J_{2}^{(1)}, \\
L^{(2)}=-J_{1}^{(2)}-J_{2}^{(2)}, & N^{(2)}=-J_{2}^{(2)}, \\
L^{(3)}=+J_{1}^{(3)}-J_{2}^{(3)}, & N^{(3)}=-J_{2}^{(3)} .
\end{array}
$$

In order to have a non-zero three-point function, the sum of the $R$-charges of the three operators must be zero:

$$
\sum_{a=1}^{3} J_{1}^{(a)}=\sum_{a=1}^{3} J_{2}^{(a)}=0 .
$$

The conservation of charges gives

$$
N^{(1)}=N^{(2)}+N^{(3)}, \quad 2 N^{(3)}=L^{(1)}+L^{(3)}-L^{(2)} .
$$

The tree-level structure constant for the case when $\mathcal{O}^{(2)}$ is a BPS field was computed in $[23,24]$, and in the general case in [26]. Here we will apply the formalism of this paper to compute the one-loop result, previously obtained in [37, 39]. Our computation agrees with that of $[37,39]$, giving the result in a concise and elegant form. The classical limit of the one loop result is taken in section 6 .

\subsection{Three-point functions at one loop}

In the language of integrable spin chains one identifies

$$
\operatorname{Tr} Z Z X X X \ldots X X Z X \quad \leftrightarrow \quad|\uparrow \uparrow \downarrow \downarrow \ldots \downarrow \uparrow \uparrow\rangle,
$$

and the three operators are in correspondence with three eigenstates $\left|\mathbf{u}^{(1)}\right\rangle,\left|\mathbf{u}^{(2)}\right\rangle$ and $\left|\mathbf{u}^{(3)}\right\rangle$ of the dilatation operator. The structure constant is expressed as [23]

$$
C_{123}\left(g^{2}\right)=\frac{\left\langle\mathbf{u}^{(1)}, \mathbf{u}^{(2)}, \mathbf{u}^{(3)}\right\rangle}{\left(\left\langle\mathbf{u}^{(1)} \mid \mathbf{u}^{(1)}\right\rangle\left\langle\mathbf{u}^{(2)} \mid \mathbf{u}^{(2)}\right\rangle\left\langle\mathbf{u}^{(3)} \mid \mathbf{u}^{(3)}\right\rangle\right)^{1 / 2}},
$$

with the cubic vertex in the numerator given by $[39]^{26}$

$$
\left\langle\mathbf{u}^{(1)}, \mathbf{u}^{(2)}, \mathbf{u}^{(3)}\right\rangle \equiv\left\langle\mathbf{u}^{(2)}\left|\mathbb{I}_{2} \mathcal{O}_{12} \mathbb{I}_{1}\right| \mathbf{u}^{(1)}\right\rangle\left\langle\uparrow^{L^{(23)}} \cdots \downarrow^{L^{(13)}} \cdots \downarrow\left|\mathbb{I}_{3}\right| \mathbf{u}^{(3)}\right\rangle .
$$

The operator $\mathcal{O}_{12}$ captures the particular structure of the contractions of the elementary fields at the splitting point, cf. figure 2 :

$$
\mathcal{O}_{12}=\sum_{i_{1} \ldots i_{L}(12)=\uparrow, \downarrow}\left|i_{1} \ldots i_{L^{(12)}} \uparrow^{L^{(23)}} \cdots\right\rangle\left\langle i_{1} \ldots i_{L^{(12)}} \downarrow^{L^{(13)}} \cdots \downarrow\right| .
$$

\footnotetext{
${ }^{26}$ We prefer to use the formulation from [39] for the three-point functions, since it is free from the complications which arise when considering "flipping".
} 


$$
\begin{array}{r}
\mathcal{O}_{12}=\sum_{i_{1} \ldots i_{L^{(12)}}=\uparrow, \downarrow}\left|i_{1} \ldots i_{L^{(12)}} \uparrow^{L^{(23)}} \cdots\right\rangle\left\langle i_{1} \ldots i_{L^{(12)}} \downarrow^{L^{(13)}} \cdots \downarrow\right| \\
\\
\quad\left\langle\uparrow^{L^{(23)}} \cdots \downarrow^{L^{(13)}} \cdots \downarrow\right|
\end{array}
$$

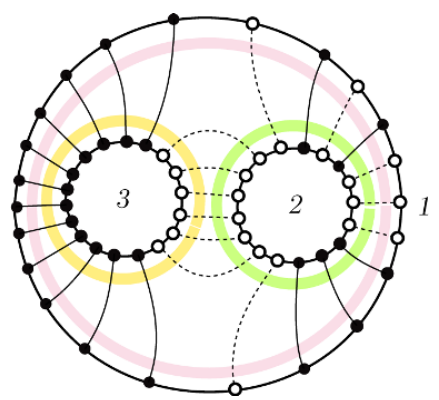

Figure 2. Structure of contractions captured by the operator $\mathcal{O}_{12}$ defined by (5.9) and by the vector $\left\langle\uparrow^{L^{(23)}} \cdots \uparrow \downarrow^{L^{(13)}} \cdots \downarrow\right|$.

The insertions $\mathbb{I}_{1,2,3}$ represent the Hamiltonian insertions and they have to be determined by perturbative gauge theory computations. Up to one-loop order they have been computed in $[22,34]$

$$
\begin{aligned}
& \mathbb{I}_{1}=1-g^{2}\left(\mathrm{H}_{L^{(12)}}^{(1)}+\mathrm{H}_{L^{(1)}}^{(1)}\right)+\ldots \\
& \mathbb{I}_{2}=1-g^{2}\left(\mathrm{H}_{L^{(12)}}^{(2)}+\mathrm{H}_{L^{(2)}}^{(2)}\right)+\ldots \\
& \mathbb{I}_{3}=1-g^{2}\left(\mathrm{H}_{L^{(31)}}^{(3)}+\mathrm{H}_{L^{(3)}}^{(3)}\right)+\ldots
\end{aligned}
$$

The knowledge of the Hamiltonian insertions at higher order is one of the main obstructions in computing the three-point function at two loop and higher. The other obstructions are to take into account the dressing factor and the wrapping contributions. Let us show now how to compute the three-point function at one loop. First, we are going to choose carefully the inhomogeneities corresponding to the three operators. Since we are splitting and joining the chains, it is convenient to have the same values of the inhomogeneities for the pieces that we are matching, i.e.

$$
\boldsymbol{\theta}^{(1)}=\boldsymbol{\theta}^{(12)} \cup \boldsymbol{\theta}^{(13)}, \quad \boldsymbol{\theta}^{(2)}=\boldsymbol{\theta}^{(12)} \cup \boldsymbol{\theta}^{(23)}, \quad \boldsymbol{\theta}^{(3)}=\boldsymbol{\theta}^{(13)} \cup \boldsymbol{\theta}^{(23)} .
$$

Moreover, we are going to choose the values of the three groups of inhomogeneities as follows

$$
\theta_{l}^{(a b)}=2 g \sin \frac{2 \pi l}{L^{(a b)}}, \quad l=1, \ldots, L^{(a b)} .
$$

This choice is compatible with the following values of the coefficients defined in (4.10) and (4.14)

$$
\begin{aligned}
& \nu_{0}^{(a b)}=\nu_{L^{(a b)}}^{(a b)}=0, \quad \rho_{0}^{(a b)}=\rho_{L^{(a b)}}^{(a b)}=0 \\
& \rho_{1}^{(a b)}=\rho_{L^{(a b)}+1}^{(a b)}=2 g^{2} .
\end{aligned}
$$

To compute the cubic vertex, we shall split each of the $\mathrm{S}^{(a)}$-operators into pieces which commute with the insertions and among themselves, $\mathrm{S}^{(a b)}$, and pieces which do not commute with the insertions and the rest, $\delta \mathrm{S}^{(a)}$,

$$
\mathrm{S}^{(1)}=\mathrm{S}^{(12)} \mathrm{S}^{(13)} \delta \mathrm{S}^{(1)}, \quad \mathrm{S}^{(2)}=\mathrm{S}^{(12)} \mathrm{S}^{(23)} \delta \mathrm{S}^{(2)}, \quad \mathrm{S}^{(3)}=\mathrm{S}^{(13)} \mathrm{S}^{(23)} \delta \mathrm{S}^{(3)} .
$$


For the one-loop three-point function, this splitting is done as follows

$$
\begin{aligned}
& \mathrm{S}^{(a b)}=\exp \left(i \sum_{k=1}^{L^{(a b)}-1} \nu_{k}^{(a b)} \mathrm{H}_{k}^{(a b)}-\frac{1}{2} \sum_{k=2}^{L^{(a b)}-1} \rho_{k}^{(a b)}[\mathrm{H}]_{k-1}^{(a b)}+\ldots\right), \\
& \delta \mathrm{S}^{(1)}=1-g^{2}\left([\mathrm{H}]_{L^{(1)}}^{(1)}+[\mathrm{H}]_{L^{(12)}}^{(1)}\right)+\ldots \\
& \delta \mathrm{S}^{(2)}=1-g^{2}\left([\mathrm{H}]_{L^{(2)}}^{(2)}+[\mathrm{H}]_{L^{(12)}}^{(2)}\right)+\ldots \\
& \delta \mathrm{S}^{(3)}=1-g^{2}\left([\mathrm{H}]_{L^{(3)}}^{(3)}+[\mathrm{H}]_{L^{(13)}}^{(3)}\right)+\ldots
\end{aligned}
$$

Inserting the split expressions into the equation (5.8), one can see that the $\mathrm{S}^{(a b)}$ parts cancel, $\mathrm{S}^{(12)}$ because it commutes with $\mathcal{O}_{12}$, and $\mathrm{S}^{(13)}$ and $\mathrm{S}^{(23)}$ because they act on totally symmetric pieces. One is left with

$$
\begin{aligned}
\left\langle\mathbf{u}^{(1)}, \mathbf{u}^{(2)}, \mathbf{u}^{(3)}\right\rangle & =\text { involved } \times \text { simple }, \\
\text { involved } & =\left\langle\mathbf{u}^{(2)} ; \boldsymbol{\theta}^{(2)}\left|\delta \mathrm{S}_{2}^{-1} \mathbb{I}_{2} \mathcal{O}_{12} \mathbb{I}_{1} \delta \mathrm{S}_{1}\right| \mathbf{u}^{(1)} ; \boldsymbol{\theta}^{(1)}\right\rangle \\
\text { simple } & =\left\langle\uparrow \ldots \uparrow \downarrow \ldots \downarrow\left|\mathbb{I}_{3} \delta \mathrm{S}_{3}\right| \mathbf{u}^{(3)} ; \boldsymbol{\theta}^{(3)}\right\rangle,
\end{aligned}
$$

where we used the notations from [39] to facilitate the comparison. The leading (tree-level) contribution to the correlator can be computed by fixing the inhomogeneities and using the freezing method of [25]. The basic idea of the freezing trick is to get a sequence of down spins by synchronizing the rapidities of a number of magnons with the same number of inhomogeneities. The simplest example is

$$
|\uparrow \ldots \uparrow \downarrow \ldots \downarrow\rangle=\mathrm{B}\left(z_{1}^{(23)}\right) \ldots \mathrm{B}\left(z_{L^{(23)}}^{(23)}\right)|\Omega\rangle=\left|\mathbf{z}^{(23)} ; \boldsymbol{\theta}^{(3)}\right\rangle \quad \text { with } \quad z_{k}^{(a b)} \equiv \theta_{k}^{(a b)}-\frac{i}{2}
$$

so that we obtain

$$
\left\langle\uparrow \ldots \uparrow \downarrow \ldots \downarrow \mid \mathbf{u}^{(3)} ; \boldsymbol{\theta}^{(3)}\right\rangle=\left\langle\mathbf{z}^{(23)} ; \boldsymbol{\theta}^{(3)} \mid \mathbf{u}^{(3)} ; \boldsymbol{\theta}^{(3)}\right\rangle
$$

and, similarly,

$$
\left\langle\mathbf{u}^{(2)} ; \boldsymbol{\theta}^{(2)}\left|\mathcal{O}_{12}\right| \mathbf{u}^{(1)} ; \boldsymbol{\theta}^{(1)}\right\rangle=\left\langle\mathbf{u}^{(2)} \cup \mathbf{z}^{(13)} ; \boldsymbol{\theta}^{(1)} \mid \mathbf{u}^{(1)} ; \boldsymbol{\theta}^{(1)}\right\rangle
$$

Both of these expressions are scalar products of a Bethe eigenstate with an off-shell vector, and as such they can be expressed in terms of Slavnov determinants. Let us point out that the two scalar products (5.18), (5.19) are no longer symmetric under the permutation of inhomogeneities $\boldsymbol{\theta}^{(3)}$ and $\boldsymbol{\theta}^{(1)}$, respectively. Instead, as we show in appendix F (see also [26]) the expression (5.18) does not depend at all on the group of inhomogeneities $\boldsymbol{\theta}^{(23)}$ but only on $\boldsymbol{\theta}^{(13)}$ and, similarly, (5.19) does not depend on $\boldsymbol{\theta}^{(13)}$ but only on $\boldsymbol{\theta}^{(12)}$.

Now let us proceed with the calculation of the one-loop corrections. The norms are computed as

$$
\left\langle\mathbf{u}^{(a)} \mid \mathbf{u}^{(a)}\right\rangle=\left\langle\mathbf{u}^{(a)}, \boldsymbol{\theta}^{(a)} \mid \mathbf{u}^{(a)}, \boldsymbol{\theta}^{(a)}\right\rangle,
$$


where the inhomogeneities on the r.h.s. are given by the values (5.11). The corrections to the cubic vertex coming from the Hamiltonian insertions and the insertions of the Soperators can be easiest evaluated by transforming them into derivatives. For this purpose, we use the relations

$$
\begin{aligned}
\mathrm{H}_{L^{(a)}}^{(a)}\left|\mathbf{u}^{(a)}, \boldsymbol{\theta}^{(a)}\right\rangle & =\left(\mathrm{Q}_{2}^{(a)}-\mathrm{D}_{L^{(a)}}^{(a)}\right)\left|\mathbf{u}^{(a)}, \boldsymbol{\theta}^{(a)}\right\rangle \\
\mathrm{H}_{L^{(a)}}^{(a)}\left|\mathbf{u}^{(a)}, \boldsymbol{\theta}^{(a)}\right\rangle & =-\mathrm{D}_{L^{(b a)}\left(\mathbf{u}^{(a)}, \boldsymbol{\theta}^{(a)}\right\rangle}^{(a)}, \\
{[\mathrm{H}]_{L^{(b a)}}^{(a)}\left|\mathbf{u}^{(a)}, \boldsymbol{\theta}^{(a)}\right\rangle } & =\left(\frac{1}{2}\left(\mathrm{D}_{L^{(b a)}}^{(a) 2}-\mathrm{D}_{L^{(b a)}+1}^{(a) 2}\right)+\left(\mathrm{D}_{L^{(b a)}}^{(a)}-\mathrm{D}_{L^{(b a)}+1}^{(a)}\right)\right)\left|\mathbf{u}^{(a)}, \boldsymbol{\theta}^{(a)}\right\rangle, \\
{[\mathrm{H}]_{L^{(a)}}^{(a)}\left|\mathbf{u}^{(a)}, \boldsymbol{\theta}^{(a)}\right\rangle } & =\left(\frac{1}{2}\left(\mathrm{D}_{L^{(a)}}^{(a) 2}-\mathrm{D}_{1}^{(a) 2}\right)+\left(\mathrm{D}_{L^{(a)}}^{(a)}-\mathrm{D}_{1}^{(a)}\right)-\delta_{\text {bound }}^{(a)}\right)\left|\mathbf{u}^{(a)}, \boldsymbol{\theta}^{(a)}\right\rangle,
\end{aligned}
$$

with

$$
\begin{aligned}
\delta_{\text {bound }}^{(a)}\left|\mathbf{u}^{(a)}, \boldsymbol{\theta}^{(a)}\right\rangle & =\left(\frac{1}{2} \mathrm{Q}_{2}^{(a) 2}+i \mathrm{Q}_{3}^{(a)}+\mathrm{P}_{1 L^{(a)}} \mathrm{Q}_{2}^{(a)}\right)\left|\mathbf{u}^{(a)}, \boldsymbol{\theta}^{(a)}\right\rangle \\
& =\left(i \mathrm{E}_{3}^{(a)}-\frac{1}{2} \mathrm{E}_{2}^{(a) 2}+\mathrm{E}_{2}^{(a)}\left(1+\mathrm{D}_{L^{(a)}}^{(a)}\right)\right)\left|\mathbf{u}^{(a)}, \boldsymbol{\theta}^{(a)}\right\rangle .
\end{aligned}
$$

Above, it is understood that the inhomogeneities are set to zero after acting with the derivatives. After performing the algebra, see appendix G, we obtain for the factor simple:

$$
\begin{aligned}
\text { simple }= & \left\langle\mathbf{z}^{(23)} ; \boldsymbol{\theta}^{(3)}\left|\mathbb{I}_{3} \delta \mathrm{S}_{3}\right| \mathbf{u}^{(3)} ; \boldsymbol{\theta}^{(3)}\right\rangle \\
= & \left\langle\mathbf{z}^{(23)} ; \boldsymbol{\theta}^{(3)} \mid \mathbf{u}^{(3)} ; \boldsymbol{\theta}^{(3)}\right\rangle \\
& +\left.g^{2}\left(\partial_{1}^{(3)} \partial_{2}^{(3)}-i \mathrm{E}_{2}^{(3)} \partial_{1}^{(3)}+i \mathrm{E}_{3}^{(3)}-\frac{1}{2} \mathrm{E}_{2}^{(3) 2}\right)\left\langle\mathbf{z}^{(23)} ; \boldsymbol{\theta}^{(3)} \mid \mathbf{u}^{(3)} ; \boldsymbol{\theta}^{(3)}\right\rangle\right|_{\theta=0}
\end{aligned}
$$

and for the factor involved:

$$
\begin{aligned}
\text { involved }= & \left\langle\mathbf{u}^{(2)} ; \boldsymbol{\theta}^{(2)}\left|\delta \mathrm{S}_{2}^{-1} \mathbb{I}_{2} \mathcal{O}_{12} \mathbb{I}_{1} \delta \mathrm{S}_{1}\right| \mathbf{u}^{(1)} ; \boldsymbol{\theta}^{(1)}\right\rangle \\
= & \left\langle\mathbf{u}^{(2)} \cup \mathbf{z}^{(13)} ; \boldsymbol{\theta}^{(1)} \mid \mathbf{u}^{(1)} ; \boldsymbol{\theta}^{(1)}\right\rangle \\
& +\left.g^{2}\left(\partial_{1}^{(1)} \partial_{2}^{(1)}-i \delta \mathrm{E}_{2} \partial_{1}^{(1)}+i \delta \mathrm{E}_{3}-\frac{1}{2} \delta \mathrm{E}_{2}^{2}\right)\left\langle\mathbf{u}^{(2)} \cup \mathbf{z}^{(13)} ; \boldsymbol{\theta}^{(1)} \mid \mathbf{u}^{(1)} ; \boldsymbol{\theta}^{(1)}\right\rangle\right|_{\theta=0}
\end{aligned}
$$

where we have used the notation $\delta \mathrm{E}_{r}=\mathrm{E}_{r}^{(1)}-\mathrm{E}_{r}^{(2)}$. In the main terms in the equations (5.22) and (5.23) the inhomogeneities are set to their BDS values (5.11), while in the last term they are set to zero. Since here we are interested only in the one-loop order, it is not important whether we set the inhomogeneities to zero or not, after taking the derivatives. The two expressions (5.22) and (5.23) look similar, the first being a particular limit of the second. When computing the three-point function, only the modulus square of the overlaps is relevant, since the phase can be always changed by a redefinition of the states. By this argument, we should drop the imaginary part in the above expressions, e.g. the terms containing $\mathrm{E}_{3}^{(a)}$ with $a=1,2,3$. Gromov and Vieira argued in [39] that the term containing $i \delta \mathrm{E}_{2} \partial_{1}^{(1)}+\frac{1}{2} \delta \mathrm{E}_{2}^{2}$ is also imaginary. By the same argument, the term containing $i \mathrm{E}_{2}^{(3)} \partial_{1}^{(3)}+\frac{1}{2} \mathrm{E}_{2}^{(3) 2}$ should be imaginary, too. 
Let us check now that our results are compatible with those of $[37,39]$. Written in our notations, their result is given by $^{27}$ (see (4.29) and appendix F)

$$
\begin{aligned}
\text { simple } & =\left(1-\frac{g^{2}}{2} \sum_{k=1}^{L^{(3)}} \mathrm{D}_{k}^{(3) 2}\right) \mathscr{A}_{\mathbf{u}^{(3)}, \boldsymbol{\theta}^{(13)}}+\ldots, \\
\text { involved } & =\left(1-\frac{g^{2}}{2} \sum_{k=1}^{L^{(1)}} \mathrm{D}_{k}^{(1) 2}\right) \mathscr{A}_{\mathbf{u}^{(1)} \cup \mathbf{u}^{(2)}, \boldsymbol{\theta}^{(12)}}+\ldots,
\end{aligned}
$$

where the dots on the r.h.s. stand for terms which are supposed to be imaginary or of higher order in $g$. The functionals $\mathscr{A}_{\mathbf{u}^{(3)}, \boldsymbol{\theta}^{(13)}}$ and $\mathscr{A}_{\mathbf{u}^{(1)} \cup \mathbf{u}^{(2)}, \boldsymbol{\theta}^{(12)}}$ are not symmetric in all the variables $\boldsymbol{\theta}^{(3)}$ and $\boldsymbol{\theta}^{(1)}$, respectively, since they do not depend at all on $\boldsymbol{\theta}^{(23)}$ and $\boldsymbol{\theta}^{(13)}$, respectively. This means that the action of the derivatives does not simply amount to the substitution of the inhomogeneities by the BDS values (5.11) (as it would be the case for symmetric functionals). The symmetry default can be cured by rewriting (5.24), (5.25) as symmetric differential operators in the variables $\boldsymbol{\theta}^{(13)}$ and $\boldsymbol{\theta}^{(12)}$ acting on the functionals $\mathscr{A}$ :

$$
\begin{aligned}
& \text { simple }=\left(1-\frac{g^{2}}{2} \sum_{k=1}^{L^{(13)}} \mathrm{D}_{k}^{(13) 2}+\frac{g^{2}}{2}\left(\mathrm{D}_{L^{(13)}}^{(13) 2}-D_{L^{(3)}}^{(3) 2}-D_{L^{(13)}}^{(3) 2}\right)\right) \mathscr{A}_{\mathbf{u}^{(3)}, \boldsymbol{\theta}^{(13)}}+\ldots \\
& =\mathscr{A}_{\mathbf{u}^{(3)}, \boldsymbol{\theta}^{(13)}}+g^{2} \partial_{1} \partial_{L^{(13)}} \mathscr{A}_{\mathbf{u}^{(3)}, \boldsymbol{\theta}^{(13)}}+\ldots \\
& \text { involved }=\left(1-\frac{g^{2}}{2} \sum_{k=1}^{L^{(12)}} \mathrm{D}_{k}^{(12) 2}+\frac{g^{2}}{2}\left(\mathrm{D}_{L^{(12)}}^{(12) 2}-D_{L^{(1)}}^{(1) 2}-D_{L^{(12)}}^{(1) 2}\right)\right) \mathscr{A}_{\mathbf{u}^{(1)} \cup \mathbf{u}^{(2)}, \boldsymbol{\theta}^{(12)}}+\ldots \\
& =\mathscr{A}_{\mathbf{u}^{(1)} \cup \mathbf{u}^{(2)}, \boldsymbol{\theta}^{(12)}}+g^{2} \partial_{1} \partial_{L^{(12)}} \mathscr{A}_{\mathbf{u}^{(1)} \cup \mathbf{u}^{(2)}, \boldsymbol{\theta}^{(12)}}+\ldots
\end{aligned}
$$

These are exactly the results in (5.22) and (5.23), up to the terms supposed to be imaginary. We conclude that our results agree with those of [37,39]. The advantage of our formulation is that we can straightforwardly take the classical limit, while in the formulation of [39] this limit is hardly possible to take, due to the complexity of the answer. Knowing the insertions at two loops would allow to compute the two-loop correlation function in the same manner as above. If the two-loop insertions are restricted to a few sites around the splitting points, then the corresponding correction would be given by terms containing four derivatives with respect to inhomogeneities around the splitting points. This kind of contribution is subdominant in the Frolov-Tseytlin limit, as we show in section 6.3. We hope to be able to report on this point in a separate work.

\section{Three-point functions in the semi-classical limit}

In this section we evaluate the one-loop three-point function obtained in the previous section, in the limit of three heavy operators, also called semi-classical or thermodynamical limit. We send $N, L \rightarrow \infty$ but the mode numbers are kept finite. In this limit, which is

\footnotetext{
${ }^{27}$ We neglected the factors $d\left(u_{j}\right) d\left(v_{j}\right)$ because they cancel with the norms in the denominator, and we dropped the superscript from $\mathscr{A}_{\mathbf{u}, \boldsymbol{\theta}}^{-}$to use just $\mathscr{A}_{\mathbf{u}, \boldsymbol{\theta}}$.
} 
interesting from the point of view of comparison with string theory, the Bethe roots arrange themselves into a small number of macroscopic strings [87, 88]. The solution of the Bethe equations in this limit is described by a Riemann surface with a finite number of cuts. ${ }^{28}$ The general finite zone solution in the $\mathfrak{s u}(2)$ sector is described by a hyperelliptic complex curve [87].

\subsection{Scalar products and norms in the semi-classical limit}

An $N$-magnon Bethe state with magnon rapidities $\mathbf{u}=\left\{u_{1}, \ldots, u_{N}\right\}$ is characterized by its quasi-momentum $p(u)$, which is defined modulo $\pi$ by (4.27). The quasi-momentum of an on-shell Bethe state satisfies $N$ conditions

$$
\left.e^{2 i p(u)}\right|_{u=u_{j}}=-1 \quad(j=1,2,, \ldots, N),
$$

which are equivalent to the Bethe equations for the roots $\mathbf{u}$. In the thermodynamical limit the quasi-momentum is given by

$$
p(u) \simeq G_{\mathbf{u}}(u)-\frac{1}{2} G_{\boldsymbol{\theta}}(u)+\pi n,
$$

with $G_{\mathbf{u}}$ and $G_{\boldsymbol{\theta}}$ being the resolvents for the magnon rapidities and the inhomogeneities,

$$
G_{\mathbf{u}}=\partial \log Q_{\mathbf{u}}, \quad G_{\boldsymbol{\theta}}=\partial \log Q_{\boldsymbol{\theta}} .
$$

The resolvent corresponding to the distribution of the rapidities (3.8) is

$$
G_{\boldsymbol{\theta}}(u)=\frac{L}{\sqrt{u^{2}-4 g^{2}}} .
$$

The semi-classical limit of the scalar product and the norm follow from that of the functional (4.30) $[26,89]^{29}$

$$
\log \mathscr{A}_{\mathbf{u}, \boldsymbol{\theta}}^{ \pm}= \pm \oint_{\mathcal{C}} \frac{d u}{2 \pi} \operatorname{Li}_{2}\left(e^{ \pm i G_{\mathbf{u}}(u) \mp i G_{\boldsymbol{\theta}}(u)}\right)+\mathcal{O}(1), \quad L \rightarrow \infty, \quad N / L \sim 1,
$$

where the contour $\mathcal{C}$ surrounds the rapidities $\mathbf{u}$ and leaves outside $\boldsymbol{\theta}$. As a consequence, the scalar product (4.29) is expressed through the sum of the two quasi-momenta:

$$
\log \left\langle\mathbf{u}^{(1)} ; \boldsymbol{\theta} \mid \mathbf{u}^{(2)} ; \boldsymbol{\theta}\right\rangle=\oint_{\mathcal{C}^{(1)} \cup \mathcal{C}^{(2)}} \frac{d u}{2 \pi} \operatorname{Li}_{2}\left(e^{i p^{(1)}(u)+i p^{(2)}(u)}\right),
$$

where the contour $\mathcal{C}^{(a)}$ surrounds the set of rapidities $\mathbf{u}^{(a)}$ and leaves outside the set of the inhomogeneities $\boldsymbol{\theta}$. In the classical limit the derivative of the quasimomentum $p^{(a)}$ is defined on a four-sheeted Riemann surface and the discrete set of points $\mathbf{u}^{(a)}$ condenses into a set of cuts on the main sheet (similarly for the set $\boldsymbol{\theta}^{(a)}$ ).

\footnotetext{
${ }^{28}$ In [87], the spectral parameter was rescaled as $u=L x$ with $x \sim 1$. We will not introduce a new rescaled variable, but will keep in mind that $u \sim L$.

${ }^{29}$ The two expressions differ by a phase factor. This also follows from the functional relation for the dilogarithm $\operatorname{Li}_{2}\left(\frac{1}{\omega}\right)=-\operatorname{Li}_{2}(\omega)-\frac{\pi^{2}}{6}-\frac{1}{2} \log ^{2}(-\omega)$.
} 
The norm of a Bethe eigenstate is obtained in the classical limit by taking $\mathbf{u}^{(1)}=$ $\mathbf{u}^{(2)}=\mathbf{u}$ in $(6.6)$ :

$$
\log \langle\mathbf{u} ; \boldsymbol{\theta} \mid \mathbf{u} ; \boldsymbol{\theta}\rangle=\oint_{\mathcal{C}} \frac{d u}{2 \pi} \operatorname{Li}_{2}\left(e^{2 i p(u)}\right),
$$

where the contour of integration surrounds the rapidities $\mathbf{u}$ and leaves outside $\boldsymbol{\theta}$. The determination of the contour is a subtle issue because of the logarithmic branch cuts starting at the points where the argument of the dilogarithm equals 1 . The contour must avoid these cuts and its choice depends on the analytic properties of the quasimomenta.

\subsection{One-loop three-point function in the semi-classical limit}

By the computation of the previous subsection, the structure constant up to two-loop corrections is given by

$$
\begin{aligned}
\left\langle\mathbf{u}^{(1)}, \mathbf{u}^{(2)}, \mathbf{u}^{(3)}\right\rangle & \equiv e^{F_{123}}=\left(1+g^{2} \hat{\Delta}\right) e^{F_{123}(\boldsymbol{\theta})}+\mathcal{O}\left(g^{4}\right), \\
F_{123}(\boldsymbol{\theta}) & \equiv \log \mathscr{A}_{\mathbf{u}^{(2)} \cup \mathbf{u}^{(1)}, \boldsymbol{\theta}^{(12)}}+\log \mathscr{A}_{\mathbf{u}^{(3)}, \boldsymbol{\theta}^{(13)}} .
\end{aligned}
$$

where the differential operator $\hat{\Delta}$ is defined as $\left(\delta \mathrm{E}_{r}=\mathrm{E}_{r}^{(1)}-\mathrm{E}_{r}^{(2)}\right)$

$$
\hat{\Delta}=\left(\partial_{1}^{(3)} \partial_{2}^{(3)}-i \mathrm{E}_{2}^{(3)} \partial_{1}^{(3)}+i \mathrm{E}_{3}^{(3)}-\frac{1}{2} \mathrm{E}_{2}^{(3) 2}\right)+\left(\partial_{1}^{(1)} \partial_{2}^{(1)}-i \delta \mathrm{E}_{2} \partial_{1}^{(1)}+i \delta \mathrm{E}_{3}-\frac{1}{2} \delta \mathrm{E}_{2}^{2}\right) .
$$

Thus the one-loop result for the structure constant is expressed in terms of the tree-level quasiclassical expression with the inhomogeneities entering as free parameters (6.6). Using the quasiclassical formula (6.5), one obtains in the thermodynamical limit

$$
\begin{aligned}
F_{123}(\boldsymbol{\theta}) \simeq & \oint_{\mathcal{C}^{(1)} \cup \mathcal{C}^{(2)}} \frac{d u}{2 \pi} \operatorname{Li}_{2}\left(e^{i p^{(1)}(u)+i p^{(2)}(u)-i q^{(3)}(u)}\right) \\
& +\oint_{\mathcal{C}^{(3)}} \frac{d u}{2 \pi} \operatorname{Li}_{2}\left(e^{i p^{(3)}(u)+i q^{(1)}(u)-i q^{(2)}(u)}\right) .
\end{aligned}
$$

Here $p^{(a)}$ are the three quasimomenta and $q^{(a)}$ are their singular parts:

$$
p^{(a)}=G_{\mathbf{u}^{(a)}}+q^{(a)} \quad q^{(a)}=-\frac{1}{2} G_{\boldsymbol{\theta}^{(a)}} \quad(a=1,2,3) .
$$

For the complete phase in (6.8) we obtain

$$
F_{123}=F_{123}(\boldsymbol{\theta})+g^{2} \delta F_{123}+\mathcal{O}\left(g^{4}\right),
$$

where the inhomogeneities in the first term on the r.h.s. are fixed to their BDS values, and the second term

$$
\delta F_{123}=\left.e^{-F_{123}(\boldsymbol{\theta})} \hat{\Delta} e^{F_{123}(\boldsymbol{\theta})}\right|_{\boldsymbol{\theta}=0}
$$

will be computed below. The first term $F_{123}(\boldsymbol{\theta})$ is an infinite series in $g^{2}$ from which only the $\mathcal{O}\left(g^{0}\right)$ and the $\mathcal{O}\left(g^{2}\right)$ terms should be retained. 
In order to evaluate $\delta F_{123}$ one should compute the derivatives in $\theta_{1,2}$ of the phase $F_{123}(\boldsymbol{\theta})$. The computation of the derivatives in $\theta_{1,2}$ is done using the representation (6.5) of the $\mathscr{A}$-functional:

$$
\begin{aligned}
\frac{\partial}{\partial \theta_{1}} \log \mathscr{A}_{\mathbf{u}, \boldsymbol{\theta}} & =-i \oint_{\mathcal{C}} \frac{d u}{2 \pi i} \frac{1}{u^{2}} \log \left(1-e^{i G_{\mathbf{u}}-i G_{\boldsymbol{\theta}}}\right), \\
\frac{\partial}{\partial \theta_{1}} \frac{\partial}{\partial \theta_{2}} \log \mathscr{A}_{\mathbf{u}, \boldsymbol{\theta}} & =-\oint_{\mathcal{C}} \frac{d u}{2 \pi i} \frac{1}{u^{4}} \frac{1}{1-e^{i G_{\mathbf{u}}-i G_{\boldsymbol{\theta}}}} .
\end{aligned}
$$

Below we will neglect the term with the second derivative, which is of order $1 / L$ compared to the other terms. Then we have

$$
\begin{aligned}
\delta F_{123}= & i \mathrm{E}_{3}^{(3)}-\frac{1}{2} \mathrm{E}_{2}^{(3) 2}+i \mathrm{E}_{3}^{(1)}-i \mathrm{E}_{3}^{(2)}-\frac{1}{2}\left(\mathrm{E}_{3}^{(1)}-\mathrm{E}_{3}^{(2)}\right)^{2} \\
& -\left(\mathrm{E}_{2}^{(1)}-\mathrm{E}_{2}^{(2)}\right) \oint_{\mathcal{C}^{(1)} \cup \mathcal{C}^{(2)}} \frac{d u / u^{2}}{2 \pi i} \log \left(1-e^{i p^{(1)}+i p^{(2)}-i q^{(3)}}\right) \\
& -\mathrm{E}_{2}^{(3)} \oint_{\mathcal{C}^{(3)}} \frac{d u / u^{2}}{2 \pi i} \log \left(1-e^{i p^{(3)}+i q^{(1)}-i q^{(2)}}\right) \\
& -\left[\oint_{\mathcal{C}^{(3)}} \frac{d u / u^{2}}{2 \pi i} \log \left(1-e^{i p^{(3)}+i q^{(1)}-i q^{(2)}}\right)\right]^{2} \\
& -\left[\oint_{\mathcal{C}^{(1)} \cup \mathcal{C}^{(2)}} \frac{d u / u^{2}}{2 \pi i} \log \left(1-e^{i p^{(1)}+i p^{(2)}-i q^{(3)}}\right)\right]^{2} .
\end{aligned}
$$

The complete result for $\log C_{123}$ is obtained by subtracting from $F_{123}+\delta F_{123}$ the logarithms of the norms of the three states, given by the contour integrals (6.7).

As we mentioned earlier, the choice of the integration contours is a non-trivial problem. The heuristic derivations of the quasiclassical limit in $[24,26]$ require that the contour of integration $\mathcal{C}^{(a)}$ encircles the cuts $\mathbf{u}^{(a)}$ and leaves outside the the $\boldsymbol{\theta}$-cut. However this prescription does not determine the contours completely because it says nothing about the logarithmic singularities of the integrand at the points where the argument of the dilogarithm takes value 1 . A necessary condition on the integration contours is that they should not cross any of the cuts produced by these singularities. In the contour integral along $\mathcal{C}^{(a)} \cup \mathcal{C}^{(b)}$ the positions of the singularities depend on the analytic properties of both $p^{(a)}$ and $p^{(b)}$. Let us denote by $\mathcal{C}^{(a b \mid c)}$ the contour which encircles the cuts $\mathbf{u}^{(a)}$ and $\mathbf{u}^{(b)}$, leaves outside the $\boldsymbol{\theta}$-cut and does not cross any of the logarithmic cuts ending at the other singularities of the integrand:

$$
\mathcal{C}^{(a)} \cup \mathcal{C}^{(b)} \rightarrow \mathcal{C}^{(a b \mid c)}
$$

In order to determine the contour of integration $\mathcal{C}^{(a b \mid c)}$, one can consider a family of solutions characterised by their global filling fractions $\alpha^{(a)}=N^{(a)} / L^{(a)}$, solve for the singular points in the limit $\alpha^{(a)} \ll 1(a=1,2,3)$ and place the contours $\mathcal{C}^{(a b \mid c)}$ so that they return to the same sheet. When $\alpha^{(a)}$ increases, the contour deforms in a continuous way.

The above rule works only if the logarithmic singularities at the points where the argument of the dilogarithm equals 1 are macroscopically far from the cuts formed by 
condensation of Bethe roots. If a singular point gets close or crosses such a cut, the integration contour should be closed on the second sheet, possibly through infinity, as in the example considered in [26].

\subsection{Comparison with the string theory results}

The semiclassical limit of the one-loop result in the SYM theory is expected to match the strong coupling result in the Frolov-Tseytlin [67] limit, where the gauge coupling $g$ is large, but the typical length $L$ is even larger, so that the effectve coupling $g^{\prime}=g / L$ is small. This is however not obvious because of the order-of-limits problem [61, 90].

The hope that such a comparison is meaningful is based on the observation that the first two orders of the expansion in $g^{\prime 2}=g^{2} / L^{2}$ of the anomalous dimension of a heavy operator in the weakly coupled gauge theory, and of the energy of the corresponding classical string state, coincide. Since the computation of the correlation function requires the knowledge of the wave functions one order beyond, it is reasonable to expect that for the three-point functions the match is to the linear order in $g^{2}$.

A string theory computation of the three-point function at strong coupling was carried out very recently by Kazama and Komatsu [46]. Kazama and Komatsu expressed the threepoint function in terms of the quasimomenta $p^{(1)}, p^{(2)}, p^{(3)}$ obtained from the monodromy matrix for a solution of the $\mathfrak{s o}(4)$ sigma model at strong coupling. They obtained for the logarithm of the structure constant an expression in terms of contour integrals, very similar to (6.11). The arguments of the dilogarithm functions are $p^{(a)}+p^{(b)}-p^{(c)}$ for $a, b, c \in\{1,2,3\}$, as well as $p^{(1)}+p^{(2)}+p^{(3)}$, and the expression is symmetric in the permutations of the three operators.

Here we will compare the Frolov-Tseytlin limit of the strong-coupling answer of [46] with the quasiclassical limit of our solution (1.4) to the linear order in $g^{\prime 2}$, assuming that the integration contours coincide, which is very likely to be the case. The main obstacle in going to two loops is that the Hamiltonian insertions have not yet been computed, although the computation seems doable and we hope to be able to report on it separately.

Let $L$ be the length scale such that $L^{(a)} / L \sim 1$ for $a=1,2,3$. The operators $\mathcal{O}^{(a)}$ correspond to solutions of the Bethe equations consisting of a few macroscopic Bethe strings. Since the typical distance between the roots forming such a string is $\sim 1$, the spectral parameter scales as $u \sim L$, which implies for the conserved charges $E_{r} \sim L^{1-r} \quad(r=1,2$, $\ldots)$. As a consequence, the correction $\delta F_{123}$ to the phase $(6.13)$ scales as $^{30}$

$$
g^{2} \delta F_{123} \sim g^{\prime 2}
$$

On the other hand, the one-loop correction in $F_{123}(\boldsymbol{\theta})$ due to the inhomogeneities, which comes from replacing $L / u \rightarrow G_{\boldsymbol{\theta}}=L / u+2 L g^{2} / u^{3}+\ldots$, scales as $g^{2} / L=L g^{\prime 2}{ }^{31}$ Therefore

\footnotetext{
${ }^{30}$ The fact that $\delta F_{123}$ does not contain a factor of $L$ in the Frolov-Tseytlin limit is not trivial in our computation presented in section 5.1, because Hamiltonian insertions scale as $L g^{\prime 2}$ and $\delta S$ also contains terms that scale as $L g^{\prime 2}$. These two contributions nicely cancel each other leaving us with a net result scaling as $g^{\prime 2}$. We suspect that similar cancellations will happen also at higher loop orders.

${ }^{31}$ The additional factor of $L$ comes from the differential $d u$ in (6.11).
} 
the correction $g^{2} \delta F_{123} / F_{123} \sim 1 / L$ can be neglected in the Frolov-Tseytlin limit and our one-loop result reads simply

$$
\left\langle\mathbf{u}^{(1)}, \mathbf{u}^{(2)}, \mathbf{u}^{(3)}\right\rangle \simeq \exp F_{123}(\boldsymbol{\theta}) .
$$

The fact that $\delta F_{123}$ disappears in the Frolov-Tseytlin limit is easy to explain: unlike the "bulk" corrections, the insertions are localised at the splitting points, and are suppressed by a factor of $1 / L$.

In the Frolov-Tseytlin limit the result of Kazama and Komatsu for $F_{123}$ (section 7.5 of $[46])$ consists of four terms,

$$
\begin{aligned}
F_{123}^{\mathrm{KK}} \simeq & \oint \operatorname{Li}_{2}\left(e^{i p^{(1)}+i p^{(2)}-i p^{(3)}}\right)+\oint \operatorname{Li}_{2}\left(e^{i p^{(3)}+i p^{(1)}-i p^{(2)}}\right) \\
& +\oint \operatorname{Li}_{2}\left(e^{i p^{(2)}+i p^{(3)}-i p^{(1)}}\right)+\oint \operatorname{Li}_{2}\left(e^{i p^{(2)}+i p^{(3)}+i p^{(1)}}\right) .
\end{aligned}
$$

Comparing this with (6.11), we see that the first two terms resemble the two terms of (6.11), while the last two terms do not have counterparts in the weak coupling result. The correspondence with the gauge theory requires that the last two terms vanish, but it is not clear if this is the case. In this paper we will discuss only the first two terms.

We will compare the first two terms (6.19) with the one-loop result (6.11). We will give an interpretation of the exponent in (6.18) in terms of the complex curves of the three heavy fields. Obviously the asymmetric form of the tree-level expression (6.11) is a consequence of the specific choice of the $\mathfrak{s u}(2)$ sectors for the three operators $\left(\mathcal{O}_{1}, \mathcal{O}_{2} \in \mathfrak{s u}(2)_{R}\right.$ and $\mathcal{O}_{3} \in$ $\left.\mathfrak{s u}(2)_{L}\right)$. Since the left and the right $\mathfrak{s u}(2)$ sectors do not talk to each other perturbatively, the dependence on the third operator factors out. This factorisation is accidental and is a consequence of the choice of the three $\mathfrak{s u}(2)$ sectors and the weak coupling limit. At strong coupling, there is no reason to expect that the three-point function factorises.

Below we are going to show that the arguments of the dilogarithm function in (6.11) are the $g / L \rightarrow 0$ limit of symmetric combinations of the three quasimomenta, e.g. $p^{(3)}+q^{(1)}-$ $q^{(2)}$ is obtained as a limit of $p^{(3)}+p^{(1)}-p^{(2)}$. For that we assume that the three operators are on-shell Bethe states from the $\mathfrak{s o}(4)$ sector. This makes sense at strong coupling when the $\mathfrak{s o}(4)$ sector is closed. ${ }^{32}$ Then the linear combination of the three quasimomenta is a meromorphic function with a four-sheeted Riemann surface as the one depicted in figure 3.

The natural parametrization of the momenta in the strong coupling limit is by the Zhukovsky variable $x$ defined by (3.7). The $a$-th quasimomentum is determined by the set

\footnotetext{
${ }^{32}$ The $\mathfrak{s o}(4)$ sectors at weak and at strong coupling have different nature and the comparison should be taken with caution, see the discussion in [91]. In the XXX spin chain (with or without inhomogeneities) the length of the chain $L=\left.\Delta\right|_{g=0}$ is expressed in terms of the two conserved $R$-charges. At perturbative level the length of an operator is conserved, since the dimension $\Delta$ of the states that contain $n$ pairs $X \bar{X}$ and have the same $R$-charges is separated by a gap $2 n$ from the states belonging to the $\mathfrak{s u}(2)$ sector and are unreachable perturbatively. On the contrary, in the sigma model there is no such gap and to the states of given charge one can add $X$ and $\bar{X}$ as constituent fields, since this combination has zero total charge. The length of a state is not a conserved charge and it is not defined at strong coupling. The $\mathfrak{s o}(4)$ sector is therefore not stable for finite $g$, but in the limit $g \rightarrow \infty$ it becomes stable again, as the $\mathfrak{s o}(4)$ sigma model is classically stable.
} 


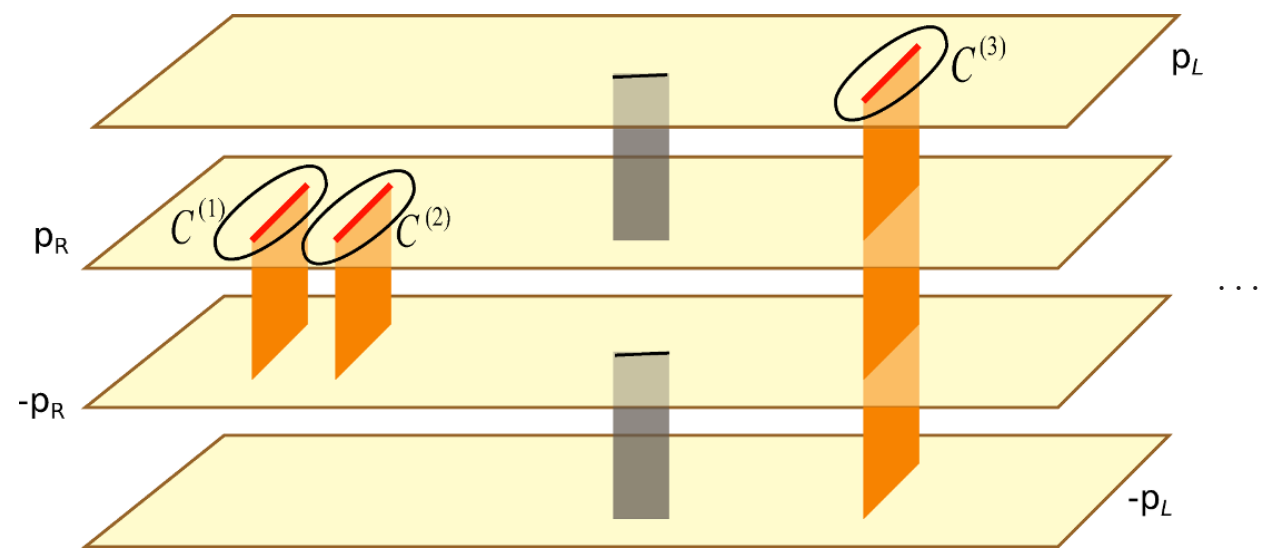

Figure 3. The Riemann surface for the three quasimomenta in the $u$-parametrization. For simplicity we assumed one-cut solutions. The left (sheets 1,4) and the right (sheets 2,3) sectors are connected by Zhukowsky cuts. In the limit $g \rightarrow 0$ the Zhukovsky cuts shrink to points and the $\mathfrak{s o}(4)$ Riemann surface decomposes into two disconnected two-sheet Riemann surfaces describing the $\mathfrak{s u}(2)_{R}$ and the $\mathfrak{s u}(2)_{L}$ sectors.

of $N^{(a)}$ rapidities $\mathbf{x}^{(a)}=\left\{x_{1}^{(a)}, \ldots, x_{N^{(a)}}^{(a)}\right\}$, which are related to the rapidities $\mathbf{u}^{(a)}$ by the Zhukovsky map (3.7). Instead of (6.12), we have

$$
p(x)=\mathcal{G}(x)-\frac{\Delta / 2}{x-g^{2} / x},
$$

where $\Delta=L+\delta$ is the conformal dimension and the resolvent

$$
\mathcal{G}(x)=\sum_{j} \frac{x_{j}^{\prime}}{x-x_{j}}, \quad x_{j}^{\prime} \equiv \frac{1}{1-g^{2} / x_{j}^{2}},
$$

is related to the resolvent in the $u$-plane by

$$
G(u)=\mathcal{G}(x)+\mathcal{G}\left(g^{2} / x\right)-\mathcal{G}(0) .
$$

The left and the right $\mathfrak{s u}(2)$ sectors in $\mathfrak{s o}(4)$ are related by the inversion symmetry $x \leftrightarrow g^{2} / x$, which exchanges right and left quasimomenta, $p_{R}$ and $p_{L}[87,92]$ :

$$
p_{R}(x)=-p_{L}\left(g^{2} / x\right)-2 \pi m, \quad m \in \mathbb{Z} .
$$

This allows to go from the four-sheeted Riemann surface in the $u$-parametrization to a two-sheet Riemann surface in the $x$-parametrization. We will use the convention

$$
p_{R}(u)=\left.p(x)\right|_{|x|>g}, \quad p_{L}(x)=\left.p(x)\right|_{|x|<g} .
$$

With this convention the left and right quasimomenta are assembled into a single quasimomentum $p(x)$ without inversion symmetry, defined on the whole $x$-plane [87]. The quasimomentum $p(x)$ is thus an analytic function defined on a hyper-elliptic Riemann surface, with poles at $x=0, x=\infty$ and at the fixed points of the inversion symmetry $x= \pm g$. 
The behavior of the quasimomentum near these poles is $[87]^{33}$

$$
p(x) \simeq\left\{\begin{array}{cl}
\left(N-\frac{1}{2} L\right) / x & (x \rightarrow \infty) \\
-\frac{1}{2} \Delta /\left(x-g^{2} / x\right) & (x \rightarrow \pm g) \\
2 \pi m+\frac{1}{2} L x / g^{2} & (x \rightarrow 0)
\end{array}\right.
$$

For the problem we are interested in, $p^{(1)}$ and $p^{(2)}$ belong to the $\mathfrak{s u}(2)_{R}$ sector, while $p^{(3)}$ belongs to the $\mathfrak{s u}(2)_{L}$ sector. Therefore the linear combinations of the type $p^{(1)}+p^{(2)}-p^{(3)}$ should be understood as

$$
\begin{aligned}
& p^{(1)}+p^{(2)}-p^{(3)} \rightarrow p^{(1)}(x)+p^{(2)}(x)+p^{(3)}\left(g^{2} / x\right) \\
& p^{(3)}+p^{(1)}-p^{(2)} \rightarrow p^{(3)}(x)-p^{(1)}\left(g^{2} / x\right)+p^{(2)}\left(g^{2} / x\right) .
\end{aligned}
$$

In the limit $g^{2} \rightarrow 0$, as it is clear from the asymptotics (6.25) of the quasimomentum at the origin, we obtain exactly the combination that appeared in the arguments of the dilogarithm in (6.11)! Since the quasimomentum appears only in the exponent, the term $2 \pi m$ can be neglected.

Now let us see if the the r.h.s. of (6.26) and the arguments of the dilogarithm in (6.11) match at linear order in $g^{\prime 2}=g^{2} / L^{2}$. This will be the case if the function $p\left(g^{2} / x\right)+q(x)$ vanishes up to $g^{\prime 4}$. We have from (6.20)

$$
\begin{aligned}
p\left(g^{2} / x\right)+q(x) & =\sum_{j=1}^{N} \frac{x_{j}^{\prime}}{g^{2} / x-x_{j}}+\frac{\Delta / 2}{x-g^{2} / x}-\frac{L / 2}{x-g^{2} / x} \\
& =2 \pi m+g^{4}\left(\frac{E_{2}}{x^{3}}-\frac{2 E_{3}}{x^{2}}\right)+\mathcal{O}\left(g^{6}\right)
\end{aligned}
$$

Therefore, if the second two terms in (6.19) can be ignored, the Frolov-Tseytlin limit of the strong coupling result from the string theory side matches, up to the subtleties related to the choice of the contour, with the one-loop result from the SYM side at order $g^{2} / L^{2}$. In any case, if the results match at tree level, they will match also at one loop. Note that if the Hamiltonian insertions at two loops are located only at the splitting points, there will be disagreement at two-loop order in the Frolov-Tseytlin limit.

We also see that the factorisation of the structure constant into two pieces, the first depending on $\mathbf{u}^{(1)}$ and $\mathbf{u}^{(2)}$ and the second depending on $\mathbf{u}^{(3)}$, takes place only in the weak coupling limit and it is a consequence of the fact that at $g \rightarrow 0$ the spectral curve for the $\mathfrak{s o}(4)$ sector splits into two components connected by a vanishing cycle (the Zhukowsky circle $|x|=g$ ). Returning to the $u$-parametrization, the three operators are defined on the Riemann surface for the $\mathfrak{s o}(4)$ sector sketched in figure 3 . The Riemann surface splits into two disjoined hyperelliptic surfaces in the limit $g \rightarrow 0$, when the two Zhukovsky cuts disappear.

\footnotetext{
${ }^{33}$ In our convention the quasimomentum has negative sign compared to [87].
} 


\section{Conclusions \& outlook}

In this work we have considered the relation between inhomogeneous and boost-induced long-range spin chains which share the same spectrum. ${ }^{34}$ We followed the philosophy that both models can be generated from a homogeneous XXX spin chain using different kinds of transformations. In one case the generators of the transformation are the boost operators studied in $[63,64]$ and the transformation can be written as a singular unitary operator $\mathrm{S}_{\mathcal{B}}$. In the other case, the transformation from a homogeneous to an inhomogeneous chain is generated by $\mathrm{S}_{\theta}$ and agrees at least up to terms of order $g^{2}$ with an inhomogeneous version of Baxter's corner transfer matrix. Since both deformations have the same spectrum, they should be related by a unitary non-singular operator $\mathrm{S}=\mathrm{S}_{\mathcal{B}} \mathrm{S}_{\theta}^{-1}$. Using the map between the two models, we have determined the scalar products of the long-range model. ${ }^{35}$ We have determined the unitary operator S up to terms of order $g^{3}$, the highest order being obtained from the comparison with the corner transfer matrix. The method works for a large class of long-range deformations of the spin $1 / 2$ XXX spin chain and can be straightforwardly extended to similar deformations of higher-rank or higher-spin models.

The map that we have discussed here is also a morphism of Yangian algebras. In particular, in the case of $\mathcal{N}=4$ super Yang-Mills theory, this morphism allows to relate the Yangian algebra of the higher loop dilatation operator to the Yangian of an inhomogeneous spin chain. A similar Yangian algebra was found for scattering amplitudes in this gauge theory (see e.g. [93] and references therein). It would be interesting to investigate whether this morphism can also be used to exploit the integrability of amplitudes at higher loops.

We have used the map between long-range and inhomogeneous spin chains in order to compute the three-point function of three operators in different $\mathfrak{s u}(2)$ sectors of $\mathcal{N}=4$ super Yang-Mills theory. The necessary ingredients are the wave functions of the dilatation operator at higher loop order, plus the diagrammatic field-theoretical corrections. These corrections have not yet been computed at two-loop order, and thus we have not performed the computation of the three-point function at two loops.

We have re-derived the results of Gromov and Viera at one loop [37, 39] at finite length, in a form that allows to straightforwardly take the semi-classical limit. In the so-called Frolov-Tseytlin limit the results of the classical limit agree with the conjecture in [38]. We have compared the one-loop computation with the strong coupling result obtained recently by Kazama and Komatsu [46] and we have found that if the results match at tree order, they match also at one loop. If the Hamiltonian insertions at two loops are located only at the splitting points, there will possibly be disagreement at two-loop order.

In order to go to three loops and beyond, one has to take into account the dressing phase as well. To include the dressing phase into this framework, we note that the generator of the corresponding long-range model $\mathrm{S}_{[\mathrm{Q} \mid \mathrm{Q}]}$ is known from the investigations in [63, 64]. For the dressing phase contributions, this operator furnishes the analogue of the boost generator $\mathrm{S}_{\mathcal{B}}$ discussed above. However, extending the correspondence to an asymptotically dual model, like the inhomogeneous spin chain, should be more involved because the values of the inhomogeneities will be state-dependent.

\footnotetext{
${ }^{34} \mathrm{Up}$ to wrapping interactions.

${ }^{35}$ The explicit expression of the operator $\mathrm{S}$ is not important for computing the scalar product.
} 


\section{Acknowledgments}

We would like to thank O. Foda, S. Komatsu, N. Gromov and M. Wheeler for stimulating discussions. We thank N. Gromov and P. Vieira for useful remarks on the initial versions of the preprint. FL would like to thank Till Bargheer for initial collaboration on studying the relation between boost operators and theta derivatives as well as for helpful discussions. FL would also like to thank Peter Orland for a discussion on corner transfer matrices. IK and DS would like to thank Melbourne University, and YJ, IK and DS would like to thank SCGP, YITP and IPMU for their warm hospitality. This work received funding from the PHC Sakura 27588UA, the European Programme IRSES UNIFY No 269217 and People Programme (Marie Curie Actions) of the European Union's Seventh Framework Programme FP7/2007-2013/ under REA Grant Agreement No 317089. The work of FL was supported by a fellowship within the Postdoc-Program of the German Academic Exchange Service (DAAD).

\section{A Inhomogeneous CTM at Order $\theta^{3}$}

It is instructive to expand the inhomogeneous CTM to order $\theta^{3}$. In the bulk we find up to terms proportional to the identity

$$
\mathcal{A}_{\theta}(0)=\exp \left[\cdots+\frac{1}{6} \sum_{k=1}^{L}\left(-\mu_{k}^{[a]} \mathrm{H}_{k}+\mu_{k}^{[b]}\left(\mathrm{H}_{k}[\mathrm{H}]_{k-1}-[\mathrm{H}]_{k-1}\right)+\mu_{k}^{[c]}[[\mathrm{H}]]_{k-1}\right)+\mathcal{O}\left(\theta^{4}\right)\right]
$$

where the individual coefficients are functions of the inhomogeneities given by

$$
\begin{aligned}
& \mu_{k}^{[a]}=-2 \sum_{x=1}^{k} \theta_{x}^{3}-2\left(\theta_{k+1}-\theta_{k}\right) \sum_{x=1}^{k} \nu_{x-1} \theta_{x}-\hat{\rho}_{k}\left(2 \theta_{k}+\theta_{k+1}\right)-\nu_{k}\left(\theta_{k}^{2}+\theta_{k} \theta_{k+1}\right), \\
& \mu_{k}^{[b]}=-2 \sum_{x=1}^{k} \theta_{x}^{3}-3 \hat{\rho}_{k} \theta_{k}-2 \nu_{k} \theta_{k}^{2} \\
& \mu_{k}^{[c]}=-2 \sum_{x=1}^{k} \theta_{x}^{3}+\left(\theta_{k+1}-\theta_{k}\right) \sum_{x=1}^{k} \nu_{x-1} \theta_{x}-\hat{\rho}_{k}\left(2 \theta_{k}+\theta_{k+1}\right)-2 \nu_{k} \theta_{k}^{2}
\end{aligned}
$$

The coefficients $\mu$ vanish for $\theta_{k}=u$ as expected.

\section{B The BDS charges from boost deformations}

Here we give explicit solutions for the BDS charges up to four loop order. We may restrict the construction introduced in $[63,64]$ to the BDS model using the above expression (3.23) for $\tau_{k}$ to find the deformation equation (3.15) for the BDS Hamiltonians:

$$
\frac{d}{d g} \overline{\mathrm{Q}}_{r}(g)=\sum_{k=1}^{\infty} 2 g^{2 k-1}\left(2 k i\left[\mathcal{B}\left[\overline{\mathrm{Q}}_{2 k+1}(g)\right], \overline{\mathrm{Q}}_{r}(g)\right]+(r+2 k-1) \overline{\mathrm{Q}}_{r+2 k}(g)\right) .
$$


Solving the above equation perturbatively one finds the following contributions at order $g^{2}, g^{4}$ and $g^{6}$ :

$$
\begin{aligned}
\overline{\mathrm{Q}}_{r}^{[2]}= & 2 i\left[\mathcal{B}\left[\overline{\mathrm{Q}}_{3}^{[0]}\right], \overline{\mathrm{Q}}_{r}^{[0]}\right]+(r+1) \overline{\mathrm{Q}}_{r+2}^{[0]}, \\
\overline{\mathrm{Q}}_{r}^{[4]}= & \frac{1}{2}\left[2 i\left[\mathcal{B}\left[\overline{\mathrm{Q}}_{3}^{[2]}\right], \overline{\mathrm{Q}}_{r}^{[0]}\right]+2 i\left[\mathcal{B}\left[\overline{\mathrm{Q}}_{3}^{[0]}\right], \overline{\mathrm{Q}}_{r}^{[2]}\right]+(r+1) \overline{\mathrm{Q}}_{r+2}^{[2]}\right. \\
& \left.+4 i\left[\mathcal{B}\left[\overline{\mathrm{Q}}_{5}^{[0]}\right], \overline{\mathrm{Q}}_{r}^{[0]}\right]+(r+3) \overline{\mathrm{Q}}_{r+4}^{[0]}\right], \\
\overline{\mathrm{Q}}_{r}^{[6]}= & \frac{1}{3}\left[2 i\left[\mathcal{B}\left[\overline{\mathrm{Q}}_{3}^{[4]}\right], \overline{\mathrm{Q}}_{r}^{[0]}\right]+2 i\left[\mathcal{B}\left[\overline{\mathrm{Q}}_{3}^{[2]}\right], \overline{\mathrm{Q}}_{r}^{[2]}\right]+2 i\left[\mathcal{B}\left[\overline{\mathrm{Q}}_{3}^{[0]}\right], \overline{\mathrm{Q}}_{r}^{[4]}\right]+(r+1) \overline{\mathrm{Q}}_{r+2}^{[4]}\right. \\
& +4 i\left[\mathcal{B}\left[\overline{\mathrm{Q}}_{5}^{[2]}\right], \overline{\mathrm{Q}}_{r}^{[0]}\right]+4 i\left[\mathcal{B}\left[\overline{\mathrm{Q}}_{5}^{[0]}\right], \overline{\mathrm{Q}}_{r}^{[2]}\right]+(r+3) \overline{\mathrm{Q}}_{r+4}^{[2]} \\
& \left.+6 i\left[\mathcal{B}\left[\overline{\mathrm{Q}}_{7}^{[0]}\right], \overline{\mathrm{Q}}_{r}^{[0]}\right]+(r+5) \overline{\mathrm{Q}}_{r+6}^{[0]}\right] .
\end{aligned}
$$

When the $\overline{\mathrm{Q}}_{r}^{[0]}$ are chosen to be the XXX charges, these expressions give the BDS Hamiltonians at two, three and four gauge theory loops.

\section{Derivation of the S-operator at order $g^{2}$}

In this appendix we compute the S-operator to higher order. We explicitly evaluate the right hand side of (4.2) to find

$$
\begin{aligned}
& \mathrm{SU}_{\theta} \mathrm{S}^{-1}= \\
& \mathrm{U}_{0}\left[1+i \sum_{k=1}^{L} \nu_{k-1} \mathrm{H}_{k}-\frac{1}{2} \sum_{k, l=1}^{L} \nu_{k-1} \nu_{l-1} \mathrm{H}_{k} \mathrm{H}_{l}-\frac{1}{2} \sum_{k=1}^{L} \rho_{k-1}[\mathrm{H}]_{k-1}\right] \\
& \times\left[1-i \sum_{k=1}^{L} \theta_{k} \mathrm{H}_{k}-\frac{1}{2} \sum_{k, l=1}^{L} \theta_{k} \theta_{l} \mathrm{H}_{k} \mathrm{H}_{l}-\frac{1}{2} \sum_{k=1}^{L} \theta_{k-1} \theta_{k}[\mathrm{H}]_{k-1}\right] \\
& \quad \times\left[1-i \sum_{k=1}^{L} \nu_{k} \mathrm{H}_{k}-\frac{1}{2} \sum_{k, l=1}^{L} \nu_{k} \nu_{l} \mathrm{H}_{k} \mathrm{H}_{l}+\frac{1}{2} \sum_{k=1}^{L} \rho_{k}[\mathrm{H}]_{k-1}\right]+\mathcal{O}\left(g^{3}\right) .
\end{aligned}
$$

Here we assumed periodicity of $\nu_{k}$ and $\rho_{k}$ to commute the shift operator with the first

bracket. Making use of the above constraints on the paramter $\nu_{k}$ that guarantee a vanishing contribution at $g^{1}$, this immediately evaluates to

$$
\begin{aligned}
\cdots=\mathrm{U}_{0}[1 & +\frac{1}{2} \sum_{k, l=1}^{L}\left(2 \nu_{k-1} \theta_{l}-2 \theta_{k} \nu_{l}+2 \nu_{k-1} \nu_{l}-\nu_{k-1} \nu_{l-1}-\nu_{k} \nu_{l}\right) \mathrm{H}_{k} \mathrm{H}_{l} \\
& \left.-\frac{1}{2} \sum_{k, l=1}^{L} \theta_{k} \theta_{l} \mathrm{H}_{k} \mathrm{H}_{l}-\frac{1}{2} \sum_{k=1}^{L} \theta_{k-1} \theta_{k}[\mathrm{H}]_{k-1}+\frac{1}{2} \sum_{k=1}^{L}\left(\rho_{k}-\rho_{k-1}\right)[\mathrm{H}]_{k-1}\right]+\mathcal{O}\left(g^{3}\right) .
\end{aligned}
$$

and using again (4.9) the first line can be simplified according to

$$
\left(2 \nu_{k-1} \theta_{l}-2 \theta_{k} \nu_{l}+2 \nu_{k-1} \nu_{l}-\nu_{k-1} \nu_{l-1}-\nu_{k} \nu_{l}\right) \mathrm{H}_{k} \mathrm{H}_{l}=\theta_{k} \theta_{l} \mathrm{H}_{k} \mathrm{H}_{l}+\nu_{k} \theta_{l}\left[\mathrm{H}_{k}, \mathrm{H}_{l}\right] .
$$

Combining the terms finally results in (4.12). 


\section{From permutations to derivatives}

In this appendix, we explain how to convert the action of any permutation operators on the monodromy matrix of Bethe states into derivatives with respect to impurities. We shall call this kind of relations PD relations. We derive the PD relations both in the bulk and at the boundary.

\section{D.1 PD relations in the bulk}

We start with algebraic Bethe ansatz. For simplicity, we choose a different normalization from the main text. The R-matrix at each site is given by

$$
\mathrm{R}_{\alpha n}^{\prime}(u)=\mathrm{I}_{\alpha n}+\frac{i}{u} \mathrm{P}_{\alpha n}, \quad n=1, \ldots, L
$$

and it is related to the one in the main text by

$$
\mathrm{R}_{\alpha \beta}^{\prime}(u)=\frac{u+i}{u} \mathrm{R}_{\alpha \beta}(u) .
$$

Here $\alpha$ denotes the auxiliary space and $n$ is the quantum space. I and $\mathrm{P}$ are identity and permutation operators, respectively. The monodromy matrix is defined as in the main text

$$
\mathrm{M}_{\alpha}(u, \boldsymbol{\theta}) \equiv \prod_{n=1}^{L} \mathrm{R}_{\alpha n}^{\prime}\left(u-\theta_{n}-i / 2\right)
$$

which becomes, in the homogeneous limit where $\theta_{k} \rightarrow 0$,

$$
\mathrm{M}_{\alpha}(u)=\prod_{n=1}^{L} \mathrm{R}_{\alpha n}^{\prime}(u-i / 2) .
$$

The authors in [37] found the following relation

$$
\left[\mathrm{P}_{k, k+1}, \mathrm{M}_{\alpha}(u)\right]=\left.i\left(\partial_{k}-\partial_{k+1}\right) \mathrm{M}_{\alpha}(u, \boldsymbol{\theta})\right|_{\boldsymbol{\theta}=0}, \quad \partial_{k} \equiv \frac{\partial}{\partial \theta_{k}},
$$

where in the r.h.s. one first takes the derivatives with respect to the impurities and then sends all impurities to zero. For simplicity, we will denote the r.h.s. of (D.5) by $i\left(\partial_{k}-\right.$ $\left.\partial_{k+1}\right) \mathrm{M}_{\alpha}(u)$ and adopt the same convention for all PD relations. As in the main text, we introduce the following notation

$$
\mathrm{D}_{k} \equiv i\left(\partial_{k}-\partial_{k+1}\right), \quad \mathrm{D}_{L}=i\left(\partial_{L}-\partial_{1}\right)
$$

We will generalize (D.5) to the case when several permutations act on the monodromy matrix. To this end, we first notice that if the action of permutation and derivatives do not overlap, they will act independently. This means, for example

$$
\partial_{j}\left[\mathrm{P}_{k, k+1}, \mathrm{M}_{\alpha}(u)\right]=\partial_{j} \mathrm{D}_{k} \mathrm{M}_{\alpha}(u), \quad \text { if } j \neq k, k+1 \text {. }
$$


The case where permutations and derivatives overlap needs to be considered more carefully. From the definition of monodromy matrix, one can derive the following relations

$$
\begin{aligned}
\partial_{k}^{n}\left[\mathrm{P}_{k, k+1}, \mathrm{M}_{\alpha}(u)\right] & =-\left(\partial_{k}^{n}-\partial_{k+1}^{n}\right) \mathrm{M}_{\alpha}(u) \mathrm{P}_{k, k+1}+\frac{1}{n+1}\left(i \partial_{k}^{n+1}-i \partial_{k+1}^{n+1}\right) \mathrm{M}_{\alpha}(u) \\
\partial_{k+1}^{n}\left[\mathrm{P}_{k, k+1}, \mathrm{M}_{\alpha}(u)\right] & =\left(\partial_{k}^{n}-\partial_{k+1}^{n}\right) \mathrm{M}_{\alpha}(u) \mathrm{P}_{k, k+1}+\frac{1}{n+1}\left(i \partial_{k}^{n+1}-i \partial_{k+1}^{n+1}\right) \mathrm{M}_{\alpha}(u) \\
\partial_{k}^{m} \partial_{k+1}^{n}\left[\mathrm{P}_{k, k+1}, \mathrm{M}_{\alpha}(u)\right] & =\frac{m ! n !}{(m+n+1) !}\left(i \partial_{k}^{m+n+1}-i \partial_{k+1}^{m+n+1}\right) \mathrm{M}_{\alpha}(u)
\end{aligned}
$$

for any $m, n \in \mathbb{N}$. Relations (D.8) can also be written as

$$
\begin{aligned}
\mathrm{P}_{k, k+1} \partial_{k+1}^{n} \mathrm{M}_{\alpha}(u) & =\partial_{k}^{n} \mathrm{M}_{\alpha}(u) \mathrm{P}_{k, k+1}+\frac{1}{n+1}\left(i \partial_{k}^{n+1}-i \partial_{k+1}^{n+1}\right) \mathrm{M}_{\alpha}(u) \\
\mathrm{P}_{k, k+1} \partial_{k}^{n} \mathrm{M}_{\alpha}(u) & =\partial_{k+1}^{n} \mathrm{M}_{\alpha}(u) \mathrm{P}_{k, k+1}+\frac{1}{n+1}\left(i \partial_{k}^{n+1}-i \partial_{k+1}^{n+1}\right) \mathrm{M}_{\alpha}(u) \\
\mathrm{P}_{k, k+1} \partial_{k}^{m} \partial_{k+1}^{n} \mathrm{M}_{\alpha}(u) & =\frac{m ! n !}{(m+n+1) !}\left(i \partial_{k}^{m+n+1}-i \partial_{k+1}^{m+n+1}\right) \mathrm{M}_{\alpha}(u)+\partial_{k}^{m} \partial_{k+1}^{n} \mathrm{M}_{\alpha}(u) \mathrm{P}_{k, k+1} .
\end{aligned}
$$

By the help of (D.9), we can derive the general PD relation. To see how this works, let us consider the following example

$$
\begin{aligned}
& {\left[\mathrm{P}_{k, k-1} \mathrm{P}_{k, k+1}, \mathrm{M}_{\alpha}(u)\right]=\left[\mathrm{P}_{k, k-1}, \mathrm{M}_{\alpha}(u)\right] \mathrm{P}_{k, k+1}+\mathrm{P}_{k, k-1}\left[\mathrm{P}_{k, k+1}, \mathrm{M}_{\alpha}(u)\right]} \\
& =\mathrm{D}_{k-1} \mathrm{M}_{\alpha}(u) \mathrm{P}_{k, k+1}+i \mathrm{P}_{k, k-1}\left(\partial_{k}-\partial_{k+1}\right) \mathrm{M}_{\alpha}(u) \\
& =\mathrm{D}_{k-1} \mathrm{M}_{\alpha}(u) \mathrm{P}_{k, k+1}+\frac{i^{2}}{2}\left(\partial_{k-1}^{2}-\partial_{k}^{2}\right) \mathrm{M}_{\alpha}(u)+i \partial_{k-1} \mathrm{M}_{\alpha}(u) \mathrm{P}_{k, k-1} \\
& \quad-i \partial_{k+1} \mathrm{M}_{\alpha}(u) \mathrm{P}_{k, k-1}-i^{2} \partial_{k+1}\left(\partial_{k-1}-\partial_{k}\right) \mathrm{M}_{\alpha}(u) \\
& =\frac{1}{2}\left(\mathrm{D}_{k-1}^{2}+2 \mathrm{D}_{k-1} \mathrm{D}_{k}\right) \mathrm{M}_{\alpha}(u)+\mathrm{D}_{k-1} \mathrm{M}_{\alpha}(u) \mathrm{P}_{k, k+1}+\left(\mathrm{D}_{k-1}+\mathrm{D}_{k}\right) \mathrm{M}_{\alpha}(u) \mathrm{P}_{k, k-1}
\end{aligned}
$$

Similarly, we can derive

$$
\begin{aligned}
& {\left[\mathrm{P}_{k, k+1} \mathrm{P}_{k, k-1}, \mathrm{M}_{\alpha}(u)\right]=} \\
& \quad=\frac{1}{2}\left(\mathrm{D}_{k}^{2}+2 \mathrm{D}_{k-1} \mathrm{D}_{k}\right) \mathrm{M}_{\alpha}(u)+\mathrm{D}_{k} \mathrm{M}_{\alpha}(u) \mathrm{P}_{k, k-1}+\left(\mathrm{D}_{k-1}+\mathrm{D}_{k}\right) \mathrm{M}_{\alpha}(u) \mathrm{P}_{k, k+1} .
\end{aligned}
$$

It is straightforward to generalize this calculation to $\left[\mathcal{P}, \mathrm{M}_{\alpha}(u)\right]$ where $\mathcal{P}$ is a product of $\mathrm{P}_{k, k+1}$. In order to apply $\mathrm{PD}$ relation on a Bethe state instead of monodromy matrix, one has also need to show the PD relation has morphism property. This means, given two functions of the monodromy matrix $\mathrm{X}(u)$ and $\mathrm{Y}(u)$, we have

$$
\left[\mathrm{P}_{k, k-1} \mathrm{P}_{k, k+1}, \mathrm{XY}\right]=\frac{1}{2}\left(\mathrm{D}_{k}^{2}+2 \mathrm{D}_{k-1} \mathrm{D}_{k}\right)(\mathrm{XY})+\mathrm{D}_{k}(\mathrm{XY}) \mathrm{P}_{k, k-1}+\left(\mathrm{D}_{k-1}+\mathrm{D}_{k}\right)(\mathrm{XY}) \mathrm{P}_{k, k+1}
$$

One can show this is true by explicit calculation. Using PD relation and morphism property we can derive the following relations, which will be useful in later discussion

$$
\begin{aligned}
\mathrm{H}_{k-1} \mathrm{H}_{k}|\mathbf{u}\rangle & =\left[\mathrm{P}_{k, k-1},\left[\mathrm{P}_{k, k+1}, \mathrm{~B}(\mathbf{u})\right]\right]|\Omega\rangle \\
& =\left[\mathrm{P}_{k, k-1} \mathrm{P}_{k, k+1}, \mathrm{~B}(\mathbf{u})\right]|\Omega\rangle-\left[\mathrm{P}_{k, k+1}, \mathrm{~B}(\mathbf{u})\right]|\Omega\rangle-\left[\mathrm{P}_{k, k-1}, \mathrm{~B}(\mathbf{u})\right]|\Omega\rangle \\
& =\frac{1}{2}\left(\mathrm{D}_{k-1}^{2}+2 \mathrm{D}_{k} \mathrm{D}_{k-1}\right)|\mathbf{u}\rangle+\mathrm{D}_{k-1}|\mathbf{u}\rangle,
\end{aligned}
$$


where we use the shorthand notation $\mathrm{B}(\mathbf{u}) \equiv \mathrm{B}\left(u_{1}\right) \cdots \mathrm{B}\left(u_{N}\right)$. Similarly, we have

$$
\mathrm{H}_{k} \mathrm{H}_{k-1}|\mathbf{u}\rangle=\frac{1}{2}\left(\mathrm{D}_{k}^{2}+2 \mathrm{D}_{k} \mathrm{D}_{k-1}\right)|\mathbf{u}\rangle+\mathrm{D}_{k}|\mathbf{u}\rangle
$$

Taking the sum and difference of (D.13) and (D.14), we obtain

$$
\begin{aligned}
{\left[\mathrm{H}_{k-1}, \mathrm{H}_{k}\right]|\mathbf{u}\rangle } & =[\mathrm{H}]_{k-1}|\mathbf{u}\rangle=\left(\frac{1}{2}\left(\mathrm{D}_{k-1}^{2}-\mathrm{D}_{k}^{2}\right)+\mathrm{D}_{k-1}-\mathrm{D}_{k}\right)|\mathbf{u}\rangle \\
\left\{\mathrm{H}_{k-1}, \mathrm{H}_{k}\right\}|\mathbf{u}\rangle & =\left(\frac{1}{2}\left(\mathrm{D}_{k-1}^{2}+\mathrm{D}_{k}^{2}\right)+\mathrm{D}_{k-1}+\mathrm{D}_{k}+2 \mathrm{D}_{k} \mathrm{D}_{k-1}\right)|\mathbf{u}\rangle .
\end{aligned}
$$

Higher order PD relations can be determined along the same lines.

\section{D.2 PD relations at the boundary}

The PD relations at the boundary are more subtle. In this section, we will derive the boundary PD relations for one and two overlapping permutations, at least one of them involving the bond $1 L$. The key observation is to notice that $\mathrm{D}_{k}$ should satisfy the following trivial constraint

$$
\sum_{k=1}^{L} \mathrm{D}_{k}=0
$$

At first order, we have

$$
\mathrm{D}_{L}|\mathbf{u}\rangle=-\sum_{k=1}^{L-1} \mathrm{D}_{k}|\mathbf{u}\rangle=\sum_{k=1}^{L-1} \mathrm{H}_{k}|\mathbf{u}\rangle=\mathrm{E}_{2}|\mathbf{u}\rangle-\mathrm{H}_{L}|\mathbf{u}\rangle,
$$

hence we find the boundary term at first order,

$$
\mathrm{H}_{L}|\mathbf{u}\rangle=-\mathrm{D}_{L}|\mathbf{u}\rangle+E_{2}|\mathbf{u}\rangle
$$

We consider now the square,

$$
\mathrm{D}_{L}^{2}=\left(\mathrm{D}_{1}+\cdots \mathrm{D}_{L-1}\right)^{2}
$$

such that

$$
\frac{1}{2}\left(\mathrm{D}_{L}^{2}-\mathrm{D}_{1}^{2}\right)|\mathbf{u}\rangle=\frac{1}{2}\left(\mathrm{D}_{2}^{2}+2 \mathrm{D}_{1} \mathrm{D}_{2}\right)|\mathbf{u}\rangle+\cdots+\frac{1}{2}\left(\mathrm{D}_{L-1}^{2}+2 \mathrm{D}_{L-2} \mathrm{D}_{L-1}\right)|\mathbf{u}\rangle+\text { non-connected }
$$

where "non-connected" are the terms $2 \mathrm{D}_{j} \mathrm{D}_{k}|\mathbf{u}\rangle$ with $|j-k| \geq 2$. Using (D.14),

$$
\frac{1}{2}\left(\mathrm{D}_{k}^{2}+2 \mathrm{D}_{k-1} \mathrm{D}_{k}\right)|\mathbf{u}\rangle=\mathrm{H}_{k} \mathrm{H}_{k-1}|\mathbf{u}\rangle-\mathrm{D}_{k}|\mathbf{u}\rangle
$$

we have

$$
\frac{1}{2}\left(\mathrm{D}_{L}^{2}-\mathrm{D}_{1}^{2}\right)|\mathbf{u}\rangle=\left(\mathrm{H}_{2} \mathrm{H}_{1}+\cdots+\mathrm{H}_{L-1} \mathrm{H}_{L-2}\right)|\mathbf{u}\rangle-\left(\mathrm{D}_{2}+\cdots \mathrm{D}_{L-1}\right)|\mathbf{u}\rangle+\text { non-connected },
$$


which is the same as

$$
\left(\frac{1}{2}\left(\mathrm{D}_{L}^{2}-\mathrm{D}_{1}^{2}\right)+\left(\mathrm{D}_{L}-\mathrm{D}_{1}\right)\right)|\mathbf{u}\rangle=\sum_{k=2}^{L-1} \mathrm{H}_{k} \mathrm{H}_{k-1}|\mathbf{u}\rangle+2 \mathrm{D}_{L}|\mathbf{u}\rangle+\text { non-connected } .
$$

Similarly, using (D.13) we have

$$
\left(\frac{1}{2}\left(\mathrm{D}_{L}^{2}-\mathrm{D}_{L-1}^{2}\right)+\left(\mathrm{D}_{L}-\mathrm{D}_{L-1}\right)\right)|\mathbf{u}\rangle=\sum_{k=2}^{L-1} \mathrm{H}_{k-1} \mathrm{H}_{k}|\mathbf{u}\rangle+2 \mathrm{D}_{L}|\mathbf{u}\rangle+\text { non-connected }
$$

Taking the difference of (D.24) and (D.25), we have

$$
\begin{aligned}
& \left(\frac{1}{2}\left(\mathrm{D}_{L}^{2}-\mathrm{D}_{1}^{2}\right)+\left(\mathrm{D}_{L}-\mathrm{D}_{1}\right)\right)|\mathbf{u}\rangle-\left(\frac{1}{2}\left(\mathrm{D}_{L}^{2}-\mathrm{D}_{L-1}^{2}\right)+\left(\mathrm{D}_{L}-\mathrm{D}_{L-1}\right)\right)|\mathbf{u}\rangle \\
& =-\sum_{k=2}^{L-1}\left[\mathrm{H}_{k-1}, \mathrm{H}_{k}\right]|\mathbf{u}\rangle=\left(2 i \mathrm{Q}_{3}+\left[\mathrm{H}_{L-1}, \mathrm{H}_{L}\right]+\left[\mathrm{H}_{L}, \mathrm{H}_{1}\right]\right)|\mathbf{u}\rangle=\left(2 i E_{3}+[\mathrm{H}]_{L-1}+[\mathrm{H}]_{L}\right)|\mathbf{u}\rangle .
\end{aligned}
$$

If we take instead the sum of (D.24) and (D.25), we have

$$
\begin{aligned}
& \left(\frac{1}{2}\left(\mathrm{D}_{L}^{2}-\mathrm{D}_{1}^{2}\right)+\left(\mathrm{D}_{L}-\mathrm{D}_{1}\right)\right)|\mathbf{u}\rangle+\left(\frac{1}{2}\left(\mathrm{D}_{L}^{2}-\mathrm{D}_{L-1}^{2}\right)+\left(\mathrm{D}_{L}-\mathrm{D}_{L-1}\right)\right)|\mathbf{u}\rangle \\
& =\sum_{k=2}^{L-1}\left\{\mathrm{H}_{k-1}, \mathrm{H}_{k}\right\}|\mathbf{u}\rangle+4 \mathrm{D}_{L}|\mathbf{u}\rangle+\text { cross terms }=\left(\sum_{k=1}^{L-1} \mathrm{H}_{k}\right)^{2}|\mathbf{u}\rangle+2 \mathrm{D}_{L}|\mathbf{u}\rangle,
\end{aligned}
$$

where we have used the fact that

$$
\sum_{k=1}^{L-1} \mathrm{H}_{k}^{2}|\mathbf{u}\rangle=2 \sum_{k=1}^{L-1} \mathrm{H}_{k}|\mathbf{u}\rangle=-2 \sum_{k=1}^{L-1} \mathrm{D}_{k}|\mathbf{u}\rangle=2 \mathrm{D}_{L}|\mathbf{u}\rangle .
$$

Now we plug $\sum_{k=1}^{L-1} \mathrm{H}_{k}=\mathrm{Q}_{2}-\mathrm{H}_{L}$ into (D.27),

$$
\begin{aligned}
& \left(\frac{1}{2}\left(\mathrm{D}_{L}^{2}-\mathrm{D}_{1}^{2}\right)+\left(\mathrm{D}_{L}-\mathrm{D}_{1}\right)\right)|\mathbf{u}\rangle+\left(\frac{1}{2}\left(\mathrm{D}_{L}^{2}-\mathrm{D}_{L-1}^{2}\right)+\left(\mathrm{D}_{L}-\mathrm{D}_{L-1}\right)\right)|\mathbf{u}\rangle= \\
& =\left(\mathrm{Q}_{2}-\mathrm{H}_{L}\right)^{2}|\mathbf{u}\rangle+2 \mathrm{D}_{L}|\mathbf{u}\rangle=\left(\mathrm{E}_{2}^{2}+2 \mathrm{E}_{2}-2 \mathrm{E}_{2} \mathrm{H}_{L}\right)|\mathbf{u}\rangle-\left[\mathrm{Q}_{2}, \mathrm{H}_{L}\right]|\mathbf{u}\rangle \\
& =\left(\mathrm{E}_{2}^{2}+2 \mathrm{E}_{2}-2 \mathrm{E}_{2} \mathrm{H}_{L}\right)|\mathbf{u}\rangle-[\mathrm{H}]_{L-1}|\mathbf{u}\rangle+[\mathrm{H}]_{L}|\mathbf{u}\rangle,
\end{aligned}
$$

where we have used (D.19). Taking the sum of (D.26) and (D.29), we find that

$$
\begin{aligned}
{[\mathrm{H}]_{L}|\mathbf{u}\rangle } & =\left(\frac{1}{2}\left(\mathrm{D}_{L}^{2}-\mathrm{D}_{1}^{2}\right)+\left(\mathrm{D}_{L}-\mathrm{D}_{1}\right)\right)|\mathbf{u}\rangle+\mathrm{E}_{2} \mathrm{H}_{L}|\mathbf{u}\rangle-C(\mathbf{u})|\mathbf{u}\rangle \\
{[\mathrm{H}]_{L-1}|\mathbf{u}\rangle } & =\left(\frac{1}{2}\left(\mathrm{D}_{L-1}^{2}-\mathrm{D}_{L}^{2}\right)+\left(\mathrm{D}_{L}-\mathrm{D}_{1}\right)\right)|\mathbf{u}\rangle-\mathrm{E}_{2} \mathrm{H}_{L}|\mathbf{u}\rangle+C(\mathbf{u})|\mathbf{u}\rangle-2 i \mathrm{E}_{3}|\mathbf{u}\rangle .
\end{aligned}
$$

where $C(\mathbf{u})$ is a function of rapidities defined by

$$
C(\mathbf{u})=\frac{1}{2}\left[\mathrm{E}_{2}^{2}(\mathbf{u})+2 \mathrm{E}_{2}(\mathbf{u})+2 i \mathrm{E}_{3}(\mathbf{u})\right] .
$$

In the derivation above, we use the fact that the second and third conserved charge read

$$
\mathrm{Q}_{2}=\sum_{k=1}^{L} \mathrm{H}_{k}, \quad \mathrm{Q}_{3}=\frac{i}{2} \sum_{k=1}^{L}[\mathrm{H}]_{k}
$$

and $\mathrm{Q}_{r}|\mathbf{u}\rangle=E_{r}|\mathbf{u}\rangle$ when $|\mathbf{u}\rangle$ is on-shell. 


\section{E From S-transformation to theta-morphism}

In this section, we will show how the theta morphism can be derived from the S-operator. Up to the order $g^{2}$, the $S$-operator reads

$$
\mathrm{S}=\exp \left(\sum_{k=1}^{L} i \nu_{k} \mathrm{H}_{k}-\frac{1}{2} \rho_{k}[\mathrm{H}]_{k-1}\right) .
$$

Let us first recall the main result of this section

$$
\mathrm{S}|\mathbf{u} ; \theta\rangle=\left(1-g^{2} E_{2} \mathrm{H}_{L}-\frac{g^{2}}{2} \sum_{k=1}^{L}\left(\mathrm{D}_{k}^{2}+2 \mathrm{D}_{k}\right)\right)|\mathbf{u}\rangle-g^{2} C|\mathbf{u}\rangle .
$$

For simplicity, we compute the action of $\mathrm{S}$ on an eigenstate $|\mathbf{u}\rangle$, but the action on a product of elements of the monodromy matrix can be computed along the same lines. The derivation of (E.2) makes use of the PD relations. At first order, we use

$$
\begin{aligned}
\mathrm{H}_{k}|\mathbf{u}\rangle & =-\mathrm{D}_{k}|\mathbf{u}\rangle, \quad k=1, \cdots L-1 \\
\mathrm{H}_{L}|\mathbf{u}\rangle & =-\mathrm{D}_{L}|\mathbf{u}\rangle+\mathrm{E}_{2}|\mathbf{u}\rangle
\end{aligned}
$$

At the second order, we use (D.15) in the bulk and (D.30) at the boundary and

$$
\mathrm{H}_{j} \mathrm{D}_{k}|\mathbf{u}\rangle=-\mathrm{H}_{j} \mathrm{H}_{k}|\mathbf{u}\rangle
$$

Now we start the derivation. The BDS eigenstate can be obtain from the homogeneous XXX state as follows

$$
|\mathbf{u}\rangle_{\mathrm{BDS}}=\mathrm{S}|\mathbf{u} ; \theta\rangle=\mathrm{S} \exp \left(\sum_{k=1}^{L} \theta_{k} \partial_{k}\right)|\mathbf{u}\rangle=\mathrm{S} \exp \left(i \sum_{k=1}^{L} \nu_{k} \mathrm{D}_{k}\right)|\mathbf{u}\rangle=\mathscr{D}_{\theta}|\mathbf{u}\rangle
$$

where $\theta_{k}$ is related to $\nu_{k}$ by the relation (4.9) with $\nu_{L}=0$, and we define the operator

$$
\mathscr{D}_{\theta} \equiv \mathrm{S} \exp \left(i \sum_{k=1}^{L} \nu_{k} \mathrm{D}_{k}\right)=1+\sum_{k=1}^{\infty} g^{k} \mathscr{D}_{\theta}^{(k)}
$$

We shall show this operator reproduce theta-morphism up to order $g^{2}$. At first order,

$$
g \mathscr{D}_{\theta}^{(1)}|\mathbf{u}\rangle=\left(i \sum_{k=1}^{L} \nu_{k} \mathrm{H}_{k}+i \sum_{k=1}^{L} \nu_{k} \mathrm{D}_{k}\right)|\mathbf{u}\rangle=\left(-i \sum_{k=1}^{L} \nu_{k} \mathrm{D}_{k}+i \sum_{k=1}^{L} \nu_{k} \mathrm{D}_{k}\right)|\mathbf{u}\rangle=0,
$$

where we have used (E.3). Hence the first order contribution vanishes. Note that by our choice of parameter $\mu_{L}=\mu_{0}=0$ hence we do not need to consider the boundary operator. As for the second order, we consider separately the non-local and local contributions,

$$
\mathscr{D}_{\theta}^{(2)}=\mathscr{D}_{\mathrm{NL}}^{(2)}+\mathscr{D}_{\mathrm{L}}^{(2)} .
$$


By non-local contribution we mean the case where two operators act independently

$$
g^{2} \mathscr{D}_{\mathrm{NL}}^{(2)}|\mathbf{u}\rangle=\sum_{|j-k| \geq 2} \nu_{j} \nu_{k}\left(-\frac{1}{2} \mathrm{H}_{j} \mathrm{H}_{k}-\frac{1}{2} \mathrm{D}_{j} \mathrm{D}_{k}+\mathrm{H}_{j} \mathrm{D}_{k}\right)|\mathbf{u}\rangle=0,
$$

hence the non-local terms do not contribute. Note that again since $\nu_{L}=0$, we do not need consider the boundary terms for non-local terms. For the local terms, we have

$$
\begin{aligned}
g^{2} \mathscr{D}_{\mathrm{L}}^{(2)}|\mathbf{u}\rangle= & -\frac{1}{2} \sum_{k=2}^{L} \rho_{k}[\mathrm{H}]_{k-1}|\mathbf{u}\rangle-\frac{1}{2} \sum_{k=1}^{L} \nu_{k} \nu_{k-1}\left\{\mathrm{H}_{k-1}, \mathrm{H}_{k}\right\}|\mathbf{u}\rangle \\
& -\sum_{k=1}^{L} \nu_{k} \nu_{k-1} \mathrm{D}_{k} \mathrm{D}_{k-1}|\mathbf{u}\rangle-\sum_{k=1}^{L} \nu_{k} \nu_{k-1}\left(\mathrm{H}_{k} \mathrm{D}_{k-1}+\mathrm{H}_{k-1} \mathrm{D}_{k}\right)|\mathbf{u}\rangle \\
& -\frac{1}{2} \sum_{k=1}^{L} \nu_{k}^{2}\left(\mathrm{H}_{k}^{2}+\mathrm{D}_{k}^{2}+2 \mathrm{H}_{k} \mathrm{D}_{k}\right)|\mathbf{u}\rangle-\frac{\rho_{1}}{2}[\mathrm{H}]_{L}|\mathbf{u}\rangle
\end{aligned}
$$

Using (E.4),

$$
\begin{aligned}
g^{2} \mathscr{D}_{\mathrm{L}}^{(2)}|\mathbf{u}\rangle= & -\frac{1}{2} \sum_{k=2}^{L} \rho_{k}[\mathrm{H}]_{k-1}|\mathbf{u}\rangle+\frac{1}{2} \sum_{k=1}^{L} \nu_{k} \nu_{k-1}\left\{\mathrm{H}_{k-1}, \mathrm{H}_{k}\right\}|\mathbf{u}\rangle \\
& -\sum_{k=1}^{L} \nu_{k} \nu_{k-1} \mathrm{D}_{k} \mathrm{D}_{k-1}|\mathbf{u}\rangle-\frac{1}{2} \sum_{k=1}^{L} \nu_{k}^{2}\left(\mathrm{D}_{k}^{2}+2 \mathrm{D}_{k}\right)|\mathbf{u}\rangle-g^{2}[\mathrm{H}]_{L}|\mathbf{u}\rangle .
\end{aligned}
$$

Now we use (D.15) to simplify the first line of (E.11),

$$
\begin{aligned}
\frac{1}{2} \sum_{k=2}^{L} \rho_{k}[\mathrm{H}]_{k-1}|\mathbf{u}\rangle & =\frac{1}{4} \sum_{k=2}^{L} \rho_{k}\left[\left(\mathrm{D}_{k-1}^{2}+2 \mathrm{D}_{k-1}\right)-\left(\mathrm{D}_{k}^{2}+2 \mathrm{D}_{k}\right)\right]|\mathbf{u}\rangle \\
& =\frac{1}{4} \sum_{k=1}^{L}\left(\rho_{k+1}-\rho_{k}\right)\left(\mathrm{D}_{k}^{2}+2 \mathrm{D}_{k}\right)|\mathbf{u}\rangle+\frac{g^{2}}{2}\left[\left(\mathrm{D}_{1}^{2}+2 \mathrm{D}_{1}\right)-\left(\mathrm{D}_{L}^{2}+2 \mathrm{D}_{L}\right)\right]|\mathbf{u}\rangle .
\end{aligned}
$$

We now use the equation (4.13) with $\tau_{3}=2 g^{2}$,

$$
\rho_{k+1}-\rho_{k}=2 g^{2}+\left(\nu_{k+1}-2 \nu_{k}+\nu_{k-1}\right) \nu_{k}
$$

that we substitute into (E.12)

$$
\begin{aligned}
-\frac{1}{2} \sum_{k=2}^{L} \rho_{k}[\mathrm{H}]_{k-1}|\mathbf{u}\rangle= & -\frac{g^{2}}{2} \sum_{k=1}^{L}\left(\mathrm{D}_{k}^{2}+2 \mathrm{D}_{k}\right)|\mathbf{u}\rangle-\frac{1}{4} \sum_{k=1}^{L}\left(\nu_{k-1} \nu_{k}+\nu_{k+1} \nu_{k}\right)\left(\mathrm{D}_{k}^{2}+2 \mathrm{D}_{k}\right)|\mathbf{u}\rangle \\
& +\frac{1}{2} \sum_{k=1}^{L} \nu_{k}^{2}\left(\mathrm{D}_{k}^{2}+2 \mathrm{D}_{k}\right)-\frac{g^{2}}{2}\left[\left(\mathrm{D}_{1}^{2}+2 \mathrm{D}_{1}\right)-\left(\mathrm{D}_{L}^{2}+2 \mathrm{D}_{L}\right)\right]|\mathbf{u}\rangle .
\end{aligned}
$$


We can also express the action of the anticommutators, using equation (D.16),

$$
\begin{aligned}
& \frac{1}{2} \sum_{k=1}^{L} \nu_{k} \nu_{k-1}\left\{\mathrm{H}_{k-1}, \mathrm{H}_{k}\right\}|\mathbf{u}\rangle \\
& =\frac{1}{4} \sum_{k=1}^{L} \nu_{k-1} \nu_{k}\left[\left(\mathrm{D}_{k-1}^{2}+2 \mathrm{D}_{k-1}\right)+\left(\mathrm{D}_{k}^{2}+2 \mathrm{D}_{k}\right)\right]|\mathbf{u}\rangle+\sum_{k=1}^{L} \nu_{k-1} \nu_{k} \mathrm{D}_{k} \mathrm{D}_{k-1}|\mathbf{u}\rangle \\
& =\frac{1}{4} \sum_{k=1}^{L}\left(\nu_{k} \nu_{k-1}+\nu_{k} \nu_{k+1}\right)\left(\mathrm{D}_{k}^{2}+2 \mathrm{D}_{k}\right)|\mathbf{u}\rangle+\sum_{k=1}^{L} \nu_{k-1} \nu_{k} \mathrm{D}_{k} \mathrm{D}_{k-1}|\mathbf{u}\rangle
\end{aligned}
$$

Last, we consider the boundary term, from (D.30),

$$
-g^{2}[\mathrm{H}]_{L}|\mathbf{u}\rangle=-\frac{g^{2}}{2}\left[\left(\mathrm{D}_{L}^{2}+2 \mathrm{D}_{L}\right)-\left(\mathrm{D}_{1}^{2}+2 \mathrm{D}_{1}\right)\right]|\mathbf{u}\rangle-g^{2} \mathrm{E}_{2} \mathrm{H}_{1, L}|\mathbf{u}\rangle-g^{2} C|\mathbf{u}\rangle .
$$

Plugging (E.14), (E.15) and (E.16) into (E.11), we obtain

$$
g^{2} \mathscr{D}_{\mathrm{L}}^{(2)}|\mathbf{u}\rangle=-\frac{g^{2}}{2} \sum_{k=1}^{L}\left(\mathrm{D}_{k}^{2}+2 \mathrm{D}_{k}\right)|\mathbf{u}\rangle-g^{2} \mathrm{E}_{2} \mathrm{H}_{1, L}|\mathbf{u}\rangle-g^{2} C|\mathbf{u}\rangle=g^{2} \mathscr{D}_{\theta}^{(2)}|\mathbf{u}\rangle,
$$

since $\mathscr{D}_{\mathrm{NL}}^{(2)}|\mathbf{u}\rangle=0$. Therefore, we have derived our main result (E.2).

\section{F Reduction formula}

In this section we prove a reduction formula for the functional $\mathscr{A}$ that we use together with the freezing method.

Reduction formula: Let $\tilde{\boldsymbol{\theta}}=\left\{\tilde{\theta}_{l}\right\}_{l=1}^{\tilde{L}}$ and $\tilde{\boldsymbol{\theta}}^{ \pm}=\left\{\tilde{\theta}_{l} \pm \frac{i}{2}\right\}_{l=1}^{\tilde{L}}$. Then

$$
\mathscr{A}_{\mathbf{u} \cup \tilde{\boldsymbol{\theta}}^{ \pm}, \boldsymbol{\theta} \cup \tilde{\boldsymbol{\theta}}}^{ \pm}=\mathscr{A}_{\mathbf{u}, \boldsymbol{\theta}}^{ \pm} .
$$

The proof is based on the representation of the scalar product (4.29) and a reduction formula for the functional $\mathscr{A}$ defined by (4.30).

Proof: By the definition (4.30),

$$
\mathscr{A}_{\mathbf{u}, \boldsymbol{\theta}}^{ \pm}=\frac{1}{\Delta_{\mathbf{u}}} \prod_{j}\left(1-\frac{Q_{\boldsymbol{\theta}}\left(u_{j} \mp \frac{i}{2}\right)}{Q_{\boldsymbol{\theta}}\left(u_{j} \pm \frac{i}{2}\right)} e^{ \pm i \partial / \partial u_{j}}\right) \Delta_{\mathbf{u}}
$$

Now compute the l.h.s., replacing in the last expression $\mathbf{u} \rightarrow \mathbf{u} \cup \tilde{\boldsymbol{\theta}}^{ \pm}$and $\boldsymbol{\theta} \rightarrow \boldsymbol{\theta} \cup \tilde{\boldsymbol{\theta}}$ :

$$
\begin{aligned}
\mathscr{A}_{\mathbf{u} \cup \tilde{\boldsymbol{\theta}}^{ \pm}, \boldsymbol{\theta} \cup \tilde{\boldsymbol{\theta}}}^{ \pm} & \frac{1}{\Delta_{\mathbf{u}} \Delta_{\tilde{\boldsymbol{\theta}}^{ \pm}} \prod_{j, l}\left(u_{j}-\tilde{\theta}_{l} \mp \frac{i}{2}\right)} \prod_{j}\left(1-\frac{Q_{\tilde{\boldsymbol{\theta}} \cup \boldsymbol{\theta}}\left(u_{j} \mp \frac{i}{2}\right)}{Q_{\tilde{\boldsymbol{\theta}} \cup \boldsymbol{\theta}}\left(u_{j} \pm \frac{i}{2}\right)} e^{ \pm i \partial / \partial u_{j}}\right) \\
& \times \prod_{l=1}^{\tilde{L}}\left(1-\frac{Q_{\tilde{\boldsymbol{\theta}} \cup \boldsymbol{\theta}}\left(\tilde{\theta}_{l}\right)}{Q_{\tilde{\boldsymbol{\theta}} \cup \boldsymbol{\theta}}\left(\tilde{\theta}_{l} \pm i\right)} e^{ \pm \partial / \partial \tilde{\theta}_{l}}\right) \Delta_{\mathbf{u}} \Delta_{\tilde{\boldsymbol{\theta}}^{ \pm}} \prod_{j, l}\left(u_{j}-\tilde{\theta}_{l} \mp \frac{i}{2}\right) .
\end{aligned}
$$


Since $Q_{\tilde{\boldsymbol{\theta}} \cup \boldsymbol{\theta}}\left(\tilde{\theta}_{l}\right)=0$, the factors containing shift operators in $\tilde{\theta}_{l}$ are equal to 1 . But then we can also remove the Vandermonds $\Delta_{\tilde{\boldsymbol{\theta}}^{ \pm}}$from both sides and write, using that $Q_{\boldsymbol{\theta}}(u-i / 2)=$ $Q_{\boldsymbol{\theta}^{ \pm}}(u)$,

$$
\begin{aligned}
\mathscr{A}_{\mathbf{u} \cup \tilde{\boldsymbol{\theta}} \pm, \boldsymbol{\theta} \cup \tilde{\boldsymbol{\theta}}}^{ \pm} & =\frac{1}{\Delta_{\mathbf{u}} \prod_{j, l}\left(u_{j}-\tilde{\theta}_{l} \mp \frac{i}{2}\right)} \prod_{j}\left(1-\frac{Q_{\tilde{\boldsymbol{\theta}} \cup \boldsymbol{\theta}}\left(u_{j} \mp \frac{i}{2}\right)}{Q_{\tilde{\boldsymbol{\theta}} \cup \boldsymbol{\theta}}\left(u_{j} \pm \frac{i}{2}\right)} e^{ \pm i \partial / \partial u_{j}}\right) \Delta_{\mathbf{u}} \prod_{j, l}\left(u_{j}-\tilde{\theta}_{l} \mp \frac{i}{2}\right) \\
& =\frac{1}{\Delta_{\mathbf{u}}} \prod_{j}\left(1-\frac{Q_{\tilde{\boldsymbol{\theta}}}\left(u_{j} \mp \frac{i}{2}\right)}{Q_{\tilde{\boldsymbol{\theta}}}\left(u_{j} \pm \frac{i}{2}\right)} \frac{Q_{\tilde{\boldsymbol{\theta}} \cup \boldsymbol{\theta}}\left(u_{j} \mp \frac{i}{2}\right)}{Q_{\tilde{\boldsymbol{\theta}} \cup \boldsymbol{\theta}}\left(u_{j} \pm \frac{i}{2}\right)} e^{ \pm i \partial / \partial u_{j}}\right) \Delta_{\mathbf{u}} \\
& =\frac{1}{\Delta_{\mathbf{u}}} \prod_{j}\left(1-\frac{Q_{\boldsymbol{\theta}}\left(u_{j} \mp \frac{i}{2}\right)}{Q_{\boldsymbol{\theta}}\left(u_{j} \pm \frac{i}{2}\right)} e^{ \pm i \partial / \partial u_{j}}\right) \Delta_{\mathbf{u}}=\mathscr{A}_{\mathbf{u}, \boldsymbol{\theta}}^{ \pm}
\end{aligned}
$$

As a consequence of the reduction formula, denoting $\mathbf{z}=\boldsymbol{\theta}^{-}$,

$$
\begin{aligned}
\left\langle\mathbf{z}^{(23)} ; \boldsymbol{\theta}^{(3)} \mid \mathbf{u}^{(3)} ; \boldsymbol{\theta}^{(3)}\right\rangle & =\mathscr{A}_{\mathbf{z}^{(23)} \cup \mathbf{u}^{(3)}, \boldsymbol{\theta}^{(3)}}=\mathscr{A}_{\mathbf{z}^{(23)} \cup \mathbf{u}^{(3)}, \boldsymbol{\theta}^{(13)} \cup \boldsymbol{\theta}^{(23)}}=\mathscr{A}_{\mathbf{u}^{(3)}, \boldsymbol{\theta}^{(13)}} ; \\
\left\langle\mathbf{u}^{(2)} \cup \mathbf{z}^{(13)} ; \boldsymbol{\theta}^{(1)} \mid \mathbf{u}^{(1)} ; \boldsymbol{\theta}^{(1)}\right\rangle & =\mathscr{A}_{\mathbf{u}^{(2)} \cup z^{(13)}, \boldsymbol{\theta}^{(1)}}=\mathscr{A}_{\mathbf{u}^{(2)} \cup z^{(13)}, \boldsymbol{\theta}^{(12)} \cup \boldsymbol{\theta}^{(13)}}=\mathscr{A}_{\mathbf{u}^{(2)}, \boldsymbol{\theta}^{(12)}} .
\end{aligned}
$$

\section{G Calculation of three-point function}

In this section, we give the details of the computation of the three- point function. We have to compute the two factors, denoted simple, respectively involved in [39],

$$
\begin{aligned}
\text { simple } & =\left\langle\uparrow \ldots \uparrow \downarrow \ldots \downarrow\left|\mathbb{I}_{3} \delta \mathrm{S}_{3}\right| \mathbf{u}^{(3)} ; \boldsymbol{\theta}^{(3)}\right\rangle, \\
\text { involved } & =\left\langle\mathbf{u}^{(2)} ; \boldsymbol{\theta}^{(2)}\left|\delta \mathrm{S}_{2}^{-1} \mathbb{I}_{2} \mathcal{O}_{12} \mathbb{I}_{1} \delta \mathrm{S}_{1}\right| \mathbf{u}^{(1)} ; \boldsymbol{\theta}^{(1)}\right\rangle .
\end{aligned}
$$

The Hamiltonian insertions $\mathbb{I}_{j}$ and the operators $\delta \mathrm{S}_{j}$ are given in equations (5.10) and (5.15), respectively. As explained in the main text, we are going to use the freezing trick, which allows to express

$$
\begin{aligned}
\left\langle\uparrow \ldots \uparrow \downarrow \ldots \downarrow \mid \mathbf{u}^{(3)} ; \boldsymbol{\theta}^{(3)}\right\rangle & =\left\langle\mathbf{z}^{(23)} ; \boldsymbol{\theta}^{(3)} \mid \mathbf{u}^{(3)} ; \boldsymbol{\theta}^{(3)}\right\rangle, \\
\left\langle\mathbf{u}^{(2)} ; \boldsymbol{\theta}^{(2)}\left|\mathcal{O}_{12}\right| \mathbf{u}^{(1)} ; \boldsymbol{\theta}^{(1)}\right\rangle & =\left\langle\mathbf{u}^{(2)} \cup \mathbf{z}^{(13)} ; \boldsymbol{\theta}^{(1)}|| \mathbf{u}^{(1)} ; \boldsymbol{\theta}^{(1)}\right\rangle .
\end{aligned}
$$

We have shown in the previous appendix that $\left\langle\mathbf{z}^{(23)} ; \boldsymbol{\theta}^{(3)} \mid \mathbf{u}^{(3)} ; \boldsymbol{\theta}^{(3)}\right\rangle$ does not depend on the inhomogeneities $\boldsymbol{\theta}^{(23)}$ and moreover it is a symmetric function of the remaining inhomogeneities $\boldsymbol{\theta}^{(13)}$. Using the equations (5.21) to transform the permutations into derivatives, we obtain

$$
\begin{aligned}
& \left\langle\mathbf{z}^{(23)} ; \boldsymbol{\theta}^{(3)}\left|\mathbb{I}_{3} \delta \mathrm{S}_{3}\right| \mathbf{u}^{(3)} ; \boldsymbol{\theta}^{(3)}\right\rangle=\left\langle\mathbf{z}^{(23)} ; \boldsymbol{\theta}^{(3)} \mid \mathbf{u}^{(3)} ; \boldsymbol{\theta}^{(3)}\right\rangle+ \\
& g^{2}\left(\mathrm{D}_{1}^{(3)}+\mathrm{D}_{L^{(13)}+1}^{(3)}+\mathrm{E}_{2}^{(3)} \mathrm{D}_{L^{(3)}}^{(3)}+i \mathrm{E}_{3}^{(3)}+\frac{1}{2}\left(\mathrm{D}_{1}^{(3) 2}+\mathrm{D}_{L^{(13)}+1}^{(3) 2}-\mathrm{D}_{L^{(3)}}^{(3) 2}-\mathrm{D}_{L^{(13)}}^{(3) 2}-\mathrm{E}_{2}^{(3) 2}\right)\right)\left\langle\mathbf{z} \mid \mathbf{u}^{(3)}\right\rangle \\
& \quad=\left\langle\mathbf{z}^{(23)} ; \boldsymbol{\theta}^{(3)} \mid \mathbf{u}^{(3)} ; \boldsymbol{\theta}^{(3)}\right\rangle+g^{2}\left(-i \mathrm{E}_{2}^{(3)} \partial_{1}^{(3)}+i \mathrm{E}_{3}^{(3)}+\partial_{1}^{(3)} \partial_{2}^{(3)}-\frac{1}{2} \mathrm{E}_{2}^{(3) 2}\right)\left\langle\mathbf{z} \mid \mathbf{u}^{(3)}\right\rangle
\end{aligned}
$$


The involved factor in (5.8) can be evaluated similarly; let us first consider

$$
\begin{aligned}
\mathcal{O}_{12} \mathbb{I}_{1} \delta \mathrm{S}_{1} \mid \mathbf{u}^{(1)} & \left.; \boldsymbol{\theta}^{(1)}\right\rangle=\mathcal{O}_{12}\left|\mathbf{u}^{(1)} ; \boldsymbol{\theta}^{(1)}\right\rangle \\
& +g^{2}\left(\mathrm{D}_{1}^{(1)}+\mathrm{D}_{L^{(12)}+1}^{(1)}+\mathrm{E}_{2}^{(1)} \mathrm{D}_{L^{(1)}}^{(1)}\right. \\
& \left.+i \mathrm{E}_{3}^{(1)}+\frac{1}{2}\left(\mathrm{D}_{1}^{(1) 2}+\mathrm{D}_{L^{(12)}+1}^{(1) 2}-\mathrm{D}_{L^{(1)}}^{(1) 2}-\mathrm{D}_{L^{(12)}}^{(1) 2}-\mathrm{E}_{2}^{(1) 2}\right)\right) \mathcal{O}^{(12)}\left|\mathbf{u}^{(1)}\right\rangle \\
= & \mathcal{O}_{12}\left|\mathbf{u}^{(1)} ; \boldsymbol{\theta}^{(1)}\right\rangle \\
& +g^{2}\left(\mathrm{D}_{1}^{(2)}+\mathrm{D}_{L^{(12)}+1}^{(2)}+\mathrm{E}_{2}^{(1)} \mathrm{D}_{L^{(2)}}^{(2)}\right. \\
& \left.+i \mathrm{E}_{3}^{(1)}+\frac{1}{2}\left(\mathrm{D}_{1}^{(2) 2}+\mathrm{D}_{L^{(12)}+1}^{(2) 2}-\mathrm{D}_{L^{(2)}}^{(2) 2}-\mathrm{D}_{L^{(12)}}^{(2) 2}-\mathrm{E}_{2}^{(1) 2}\right)\right) \mathcal{O}_{12}\left|\mathbf{u}^{(1)}\right\rangle
\end{aligned}
$$

In the last line we have used that $\mathcal{O}_{12}|\mathbf{v}\rangle$ does not depend neither on $\boldsymbol{\theta}^{(23)}$ nor on $\boldsymbol{\theta}^{(13)}$, so we can replace the derivatives $\mathrm{D}_{k}^{(1)}$ by $\mathrm{D}_{k}^{(2)}$. Similarly, we obtain for the action on the bra vector

$$
\begin{aligned}
& \left\langle\mathbf{u}^{(2)} ; \boldsymbol{\theta}^{(2)}\right| \delta \mathrm{S}_{2}^{-1} \mathbb{I}_{2}=\left\langle\mathbf{u}^{(2)} ; \boldsymbol{\theta}^{(2)}\right|+ \\
& g^{2}\left[-\mathrm{D}_{1}^{(2)}-\mathrm{D}_{L^{(12)}+1}^{(2)}-\mathrm{E}_{2}^{(2)} \mathrm{D}_{L^{(2)}}^{(2)}-i \mathrm{E}_{3}^{(2)}+\frac{1}{2}\left(\mathrm{D}_{1}^{(2) 2}+\mathrm{D}_{L^{(12)}+1}^{(2) 2}-\mathrm{D}_{L^{(2)}}^{(2) 2}-\mathrm{D}_{L^{(12)}}^{(2) 2}-\mathrm{E}_{2}^{(2) 2}\right)\right]\left\langle\mathbf{u}^{(1)}\right|,
\end{aligned}
$$

where we used that the action of the operators $\mathrm{D}_{k}$ on the left vectors is $\left(\mathrm{D}_{k}|\mathbf{u}\rangle\right)^{\dagger}=-\mathrm{D}_{k}\langle\mathbf{u}|$. Using the Leibniz rule, we have $\mathrm{D}_{k}(\langle\mathbf{u} \mid \mathbf{v}\rangle)=\left\langle\mathbf{u}\left|\mathrm{D}_{k}\right| \mathbf{v}\right\rangle+\mathrm{D}_{k}(\langle\mathbf{u}|)|\mathbf{v}\rangle$. This quantity is zero unless $k=L^{(a)}$ or $L^{(a b)}$, for the type of vectors we use in this section. We also have

$$
D_{k}^{2}(\langle\mathbf{u} \mid \mathbf{v}\rangle)=D_{k}^{2}(\langle\mathbf{u}|)|\mathbf{v}\rangle+\langle\mathbf{u}| D_{k}^{2}(|\mathbf{v}\rangle)+2 D_{k}(\langle\mathbf{u}|) D_{k}(|\mathbf{v}\rangle),
$$

and $2 D_{k}(\langle\mathbf{u}|) D_{k}(|\mathbf{v}\rangle)=4\left\langle\mathbf{u}\left|D_{k}\right| \mathbf{v}\right\rangle$. For $k=L^{(12)}$ we use that

$$
D_{L^{(12)}}^{2}\left(\left\langle\mathbf{u}\left|\mathcal{O}_{12}\right| \mathbf{v}\right\rangle\right)=D_{L^{(12)}}^{(2) 2}(\langle\mathbf{u}|) \mathcal{O}_{12}|\mathbf{v}\rangle+\langle\mathbf{u}| D_{L^{(12)}}^{(1) 2}(|\mathbf{v}\rangle)-2\left\langle\mathbf{u}\left|\mathrm{H}_{L^{(12)}}^{(2)} \mathcal{O}_{12} \mathrm{H}_{L^{(12)}}^{(1)}\right| \mathbf{v}\right\rangle
$$

with the last term being zero because $\mathrm{H}_{L^{(12)}}^{(2)} \mathcal{O}_{12} \mathrm{H}_{L^{(12)}}^{(1)}=0$, as noticed already in [39]. For $k=L^{(2)}$ one has

$$
D_{L^{(2)}}^{2}\left(\left\langle\mathbf{u}\left|\mathcal{O}_{12}\right| \mathbf{v}\right\rangle\right)=D_{L^{(2)}}^{(2) 2}(\langle\mathbf{u}|) \mathcal{O}_{12}|\mathbf{v}\rangle+\langle\mathbf{u}| D_{L^{(1)}}^{(1) 2}(|\mathbf{v}\rangle)-2\left\langle\mathbf{u}\left|\mathrm{D}_{L^{(2)}}^{(2)} \mathcal{O}_{12} \mathrm{D}_{L^{(1)}}^{(1)}\right| \mathbf{v}\right\rangle
$$

Proceeding as previously, we get

$$
0=\left\langle\mathbf{u}\left|\mathrm{H}_{L^{(2)}}^{(2)} \mathcal{O}_{12} \mathrm{H}_{L_{1}}^{(1)}\right| \mathbf{v}\right\rangle=\left\langle\mathbf{u}\left|\left(E_{2}^{(2)}-\mathrm{D}_{L^{(2)}}^{(2)}\right) \mathcal{O}_{12}\left(E_{2}^{(1)}-\mathrm{D}_{L_{1}}^{(1)}\right)\right| \mathbf{v}\right\rangle
$$

so that

$$
\left\langle\mathbf{u}\left|\mathrm{D}_{L_{2}}^{(2)} \mathcal{O}_{12} \mathrm{D}_{L_{1}}^{(1)}\right| \mathbf{v}\right\rangle=\left\langle\mathbf{u}\left|\left(E_{2}^{(2)}+E_{2}^{(1)}\right) \mathrm{D}_{L_{2}}^{(2)} \mathcal{O}_{12}\right| \mathbf{v}\right\rangle-E_{2}^{(2)} E_{2}^{(1)}\left\langle\mathbf{u}\left|\mathcal{O}_{12}\right| \mathbf{v}\right\rangle
$$

Putting together the various identities above, we obtain for involved

$$
\begin{aligned}
\left\langle\mathbf{u}^{(2)} ; \boldsymbol{\theta}^{(2)}\right| \delta \mathrm{S}_{2}^{-1} & \mathbb{I}_{2} \mathcal{O}_{12} \mathbb{I}_{1} \delta \mathrm{S}_{1}\left|\mathbf{u}^{(1)} ; \boldsymbol{\theta}^{(1)}\right\rangle=\left\langle\mathbf{u}^{(2)} ; \boldsymbol{\theta}^{(2)}\left|\mathcal{O}_{12}\right| \mathbf{u}^{(1)} ; \boldsymbol{\theta}^{(1)}\right\rangle \\
& +\frac{g^{2}}{2}\left(\mathrm{D}_{1}^{(2) 2}+\mathrm{D}_{L^{(12)}+1}^{(2) 2}-\mathrm{D}_{L^{(2)}}^{(2) 2}-\mathrm{D}_{L^{(12)}}^{(2) 2}\right. \\
& -\left(\mathrm{E}_{2}^{(1)}-\mathrm{E}_{2}^{(2)}\right)^{2}+i\left(\mathrm{E}_{3}^{(1)}-\mathrm{E}_{3}^{(2)}\right)\left\langle\mathbf{u}^{(2)} ; \boldsymbol{\theta}^{(2)}\left|\mathcal{O}_{12}\right| \mathbf{u}^{(1)} ; \boldsymbol{\theta}^{(1)}\right\rangle .
\end{aligned}
$$


Since the scalar product $\left\langle\mathbf{u}^{(2)} ; \boldsymbol{\theta}^{(2)}\left|\mathcal{O}_{12}\right| \mathbf{u}^{(1)} ; \boldsymbol{\theta}^{(1)}\right\rangle$ does not depend on the inhomogeneities $\boldsymbol{\theta}^{(13)}$ or $\boldsymbol{\theta}^{(23)}$ and is a symmetric function of the inhomogeneities $\boldsymbol{\theta}^{(12)}$, one can write

$$
\begin{aligned}
\left\langle\mathbf{u}^{(2)} ; \boldsymbol{\theta}^{(2)}\right| & \delta \mathrm{S}_{2}^{-1} \mathbb{I}_{2} \mathcal{O}_{12} \mathbb{I}_{1} \delta \mathrm{S}_{1}\left|\mathbf{u}^{(1)} ; \boldsymbol{\theta}^{(1)}\right\rangle=\left\langle\mathbf{u}^{(2)} ; \boldsymbol{\theta}^{(2)}\left|\mathcal{O}_{12}\right| \mathbf{u}^{(1)} ; \boldsymbol{\theta}^{(1)}\right\rangle \\
+ & \frac{g^{2}}{2}\left(2 \partial_{1} \partial_{2}-\left(\mathrm{E}_{2}^{(1)}-\mathrm{E}_{2}^{(2)}\right)^{2}+i\left(\mathrm{E}_{3}^{(1)}-\mathrm{E}_{3}^{(2)}\right)\right)\left\langle\mathbf{u}^{(2)} ; \boldsymbol{\theta}^{(2)}\left|\mathcal{O}_{12}\right| \mathbf{u}^{(1)} ; \boldsymbol{\theta}^{(1)}\right\rangle .
\end{aligned}
$$

This finishes our derivation of the three-point function at one loop.

Open Access. This article is distributed under the terms of the Creative Commons Attribution License (CC-BY 4.0), which permits any use, distribution and reproduction in any medium, provided the original author(s) and source are credited.

\section{References}

[1] N. Beisert et al., Review of AdS/CFT Integrability: An Overview, Lett. Math. Phys. 99 (2012) 3 [arXiv:1012.3982] [INSPIRE].

[2] J.M. Maldacena, The large- $N$ limit of superconformal field theories and supergravity, Adv. Theor. Math. Phys. 2 (1998) 231 [Int. J. Theor. Phys. 38 (1999) 1113] [hep-th/9711200] [INSPIRE].

[3] J.M. Drummond, J.M. Henn and J. Plefka, Yangian symmetry of scattering amplitudes in $N=4$ super Yang-Mills theory, JHEP 05 (2009) 046 [arXiv:0902.2987] [INSPIRE].

[4] T. Bargheer, N. Beisert, W. Galleas, F. Loebbert and T. McLoughlin, Exacting $N=4$ Superconformal Symmetry, JHEP 11 (2009) 056 [arXiv:0905.3738] [INSPIRE].

[5] G. Korchemsky and E. Sokatchev, Superconformal invariants for scattering amplitudes in N =4 SYM theory, Nucl. Phys. B $\mathbf{8 3 9}$ (2010) 377 [arXiv:1002.4625] [INSPIRE].

[6] J. Drummond, L. Ferro and É. Ragoucy, Yangian symmetry of light-like Wilson loops, JHEP 11 (2011) 049 [arXiv: 1011.4264] [INSPIRE].

[7] N. Beisert, J. Henn, T. McLoughlin and J. Plefka, One-Loop Superconformal and Yangian Symmetries of Scattering Amplitudes in $N=4$ Super Yang-Mills, JHEP 04 (2010) 085 [arXiv: 1002.1733] [INSPIRE].

[8] L.F. Alday, J. Maldacena, A. Sever and P. Vieira, Y-system for Scattering Amplitudes, J. Phys. A 43 (2010) 485401 [arXiv:1002.2459] [inSPIRE].

[9] S. Caron-Huot and S. He, Jumpstarting the All-Loop S-matrix of Planar $N=4$ Super Yang-Mills, JHEP 07 (2012) 174 [arXiv:1112.1060] [INSPIRE].

[10] D. Correa, J. Maldacena and A. Sever, The quark anti-quark potential and the cusp anomalous dimension from a TBA equation, JHEP 08 (2012) 134 [arXiv:1203.1913] [INSPIRE].

[11] N. Drukker, Integrable Wilson loops, JHEP 10 (2013) 135 [arXiv:1203.1617] [INSPIRE].

[12] A. Sever, P. Vieira and T. Wang, From Polygon Wilson Loops to Spin Chains and Back, JHEP 12 (2012) 065 [arXiv:1208.0841] [INSPIRE].

[13] L. Ferro, T. Lukowski, C. Meneghelli, J. Plefka and M. Staudacher, Spectral parameters for scattering amplitudes in $N=4$ super Yang-Mills theory, JHEP 01 (2014) 094 [arXiv:1308.3494] [INSPIRE]. 
[14] D. Chicherin, S. Derkachov and R. Kirschner, Yang-Baxter operators and scattering amplitudes in $N=4$ super-Yang-Mills theory, Nucl. Phys. B 881 (2014) 467 [arXiv: 1309.5748] [INSPIRE].

[15] D. Müller, H. Münkler, J. Plefka, J. Pollok and K. Zarembo, Yangian Symmetry of smooth Wilson Loops in $\mathcal{N}=4$ super Yang-Mills Theory, JHEP 11 (2013) 081 [arXiv:1309.1676] [INSPIRE].

[16] B. Basso, A. Sever and P. Vieira, Space-time S-matrix and Flux-tube S-matrix at Finite Coupling, Phys. Rev. Lett. 111 (2013) 091602 [arXiv:1303.1396] [INSPIRE].

[17] B. Basso, A. Sever and P. Vieira, Space-time S-matrix and Flux tube S-matrix II. Extracting and Matching Data, JHEP 01 (2014) 008 [arXiv:1306.2058] [INSPIRE].

[18] H. Elvang and Y.-t. Huang, Scattering Amplitudes, arXiv:1308.1697 [INSPIRE].

[19] S. Lee, S. Minwalla, M. Rangamani and N. Seiberg, Three point functions of chiral operators in $D=4, \mathcal{N}=4 S Y M$ at large- $N$, Adv. Theor. Math. Phys. 2 (1998) 697 [hep-th/9806074] [INSPIRE].

[20] D.Z. Freedman, S.D. Mathur, A. Matusis and L. Rastelli, Correlation functions in the $C F T(d) / A d S(d+1)$ correspondence, Nucl. Phys. B 546 (1999) 96 [hep-th/9804058] [INSPIRE].

[21] R. Roiban and A. Volovich, Yang-Mills correlation functions from integrable spin chains, JHEP 09 (2004) 032 [hep-th/0407140] [INSPIRE].

[22] K. Okuyama and L.-S. Tseng, Three-point functions in $N=4$ SYM theory at one-loop, JHEP 08 (2004) 055 [hep-th/0404190] [INSPIRE].

[23] J. Escobedo, N. Gromov, A. Sever and P. Vieira, Tailoring three-point functions and integrability, JHEP 09 (2011) 028 [arXiv: 1012.2475] [INSPIRE].

[24] N. Gromov, A. Sever and P. Vieira, Tailoring three-point functions and integrability III. Classical tunneling, JHEP 07 (2012) 044 [arXiv: 1111.2349] [INSPIRE].

[25] O. Foda, $N=4$ SYM structure constants as determinants, JHEP 03 (2012) 096 [arXiv:1111.4663] [INSPIRE].

[26] I. Kostov, Three-point function of semiclassical states at weak coupling, J. Phys. A 45 (2012) 494018 [arXiv: 1205.4412] [InSPIRE].

[27] J. Escobedo, N. Gromov, A. Sever and P. Vieira, Tailoring three-point functions and integrability II. Weak/strong coupling match, JHEP 09 (2011) 029 [arXiv:1104.5501] [INSPIRE].

[28] M. Wheeler, Multiple integral formulae for the scalar product of on-shell and off-shell Bethe vectors in SU(3)-invariant models, Nucl. Phys. B 875 (2013) 186 [arXiv:1306.0552] [INSPIRE].

[29] O. Foda, Y. Jiang, I. Kostov and D. Serban, A tree-level 3-point function in the SU(3)-sector of planar $N=4$ SYM, JHEP 10 (2013) 138 [arXiv:1302.3539] [INSPIRE].

[30] G. Georgiou, SL(2) sector: weak/strong coupling agreement of three-point correlators, JHEP 09 (2011) 132 [arXiv:1107.1850] [INSPIRE].

[31] V. Kazakov and E. Sobko, Three-point correlators of twist-2 operators in $N=4 S Y M$ at Born approximation, JHEP 06 (2013) 061 [arXiv:1212.6563] [INSPIRE].

[32] E. Sobko, A new representation for two- and three-point correlators of operators from sl(2) sector, arXiv: 1311.6957 [INSPIRE]. 
[33] P. Vieira and T. Wang, Tailoring Non-Compact Spin Chains, arXiv:1311.6404 [INSPIRE].

[34] L.F. Alday, J.R. David, E. Gava and K. Narain, Structure constants of planar $N=4$ Yang-Mills at one loop, JHEP 09 (2005) 070 [hep-th/0502186] [INSPIRE].

[35] J. Plefka and K. Wiegandt, Three-point functions of twist-two operators in $N=4$ SYM at one loop, JHEP 10 (2012) 177 [arXiv: 1207.4784] [INSPIRE].

[36] G. Georgiou, V. Gili, A. Grossardt and J. Plefka, Three-point functions in planar $\mathcal{N}=4$ super Yang-Mills Theory for scalar operators up to length five at the one-loop order, JHEP 04 (2012) 038 [arXiv: 1201.0992] [INSPIRE].

[37] N. Gromov and P. Vieira, Quantum Integrability for Three-Point Functions, Phys. Rev. Lett. 111 (2013) 211601 [arXiv:1202.4103] [INSPIRE].

[38] D. Serban, A note on the eigenvectors of long-range spin chains and their scalar products, JHEP 01 (2013) 012 [arXiv: 1203.5842] [INSPIRE].

[39] N. Gromov and P. Vieira, Tailoring three-point functions and integrability IV. Theta-morphism, arXiv: 1205.5288 [INSPIRE].

[40] A. Bissi, T. Harmark and M. Orselli, Holographic 3-point function at one loop, JHEP 02 (2012) 133 [arXiv:1112.5075] [INSPIRE].

[41] R.A. Janik, P. Surowka and A. Wereszczynski, On correlation functions of operators dual to classical spinning string states, JHEP 05 (2010) 030 [arXiv: 1002.4613] [INSPIRE].

[42] R.A. Janik and A. Wereszczynski, Correlation functions of three heavy operators: The AdS contribution, JHEP 12 (2011) 095 [arXiv: 1109.6262] [INSPIRE].

[43] Y. Kazama and S. Komatsu, On holographic three point functions for GKP strings from integrability, JHEP 01 (2012) 110 [Erratum ibid. 1206 (2012) 150] [arXiv:1110.3949] [INSPIRE].

[44] Y. Kazama and S. Komatsu, Wave functions and correlation functions for GKP strings from integrability, JHEP 09 (2012) 022 [arXiv:1205.6060] [INSPIRE].

[45] J. Caetano and J. Toledo, $\chi$-Systems for Correlation Functions, arXiv:1208.4548 [INSPIRE].

[46] Y. Kazama and S. Komatsu, Three-point functions in the $\mathrm{SU}(2)$ sector at strong coupling, JHEP 03 (2014) 052 [arXiv:1312.3727] [INSPIRE].

[47] K. Zarembo, Holographic three-point functions of semiclassical states, JHEP 09 (2010) 030 [arXiv: 1008.1059] [INSPIRE].

[48] M.S. Costa, R. Monteiro, J.E. Santos and D. Zoakos, On three-point correlation functions in the gauge/gravity duality, JHEP 11 (2010) 141 [arXiv:1008.1070] [INSPIRE].

[49] E. Buchbinder and A. Tseytlin, On semiclassical approximation for correlators of closed string vertex operators in AdS/CFT, JHEP 08 (2010) 057 [arXiv: 1005.4516] [INSPIRE].

[50] A. Bissi, C. Kristjansen, D. Young and K. Zoubos, Holographic three-point functions of giant gravitons, JHEP 06 (2011) 085 [arXiv:1103.4079] [INSPIRE].

[51] E. Buchbinder and A. Tseytlin, Semiclassical correlators of three states with large $S^{5}$ charges in string theory in $A d S_{5} \times S^{5}$, Phys. Rev. D 85 (2012) 026001 [arXiv:1110.5621] [INSPIRE].

[52] T. Klose and T. McLoughlin, A light-cone approach to three-point functions in $A d S_{5} \times S^{5}$, JHEP 04 (2012) 080 [arXiv:1106.0495] [INSPIRE].

[53] J.A. Minahan, Holographic three-point functions for short operators, JHEP 07 (2012) 187 [arXiv: 1206.3129] [INSPIRE]. 
[54] T. Bargheer, J.A. Minahan and R. Pereira, Computing Three-Point Functions for Short Operators, arXiv: 1311.7461 [INSPIRE].

[55] M.S. Costa, J. Penedones, D. Poland and S. Rychkov, Spinning conformal correlators, JHEP 11 (2011) 071 [arXiv:1107.3554] [INSPIRE].

[56] J. Caetano and J. Escobedo, On four-point functions and integrability in $N=4$ SYM: from weak to strong coupling, JHEP 09 (2011) 080 [arXiv:1107.5580] [INSPIRE].

[57] L.F. Alday and A. Bissi, Higher-spin correlators, JHEP 10 (2013) 202 [arXiv:1305.4604] [INSPIRE].

[58] N. Beisert, V. Dippel and M. Staudacher, A novel long range spin chain and planar $N=4$ super Yang-Mills, JHEP 07 (2004) 075 [hep-th/0405001] [INSPIRE].

[59] A. Rej, D. Serban and M. Staudacher, Planar $N=4$ gauge theory and the Hubbard model, JHEP 03 (2006) 018 [hep-th/0512077] [INSPIRE].

[60] D. Bernard, M. Gaudin, F. Haldane and V. Pasquier, Yang-Baxter equation in long range interacting system, J. Phys. A 26 (1993) 5219 [INSPIRE].

[61] D. Serban and M. Staudacher, Planar $N=4$ gauge theory and the Inozemtsev long range spin chain, JHEP 06 (2004) 001 [hep-th/0401057] [INSPIRE].

[62] D. Serban, Eigenvectors and scalar products for long range interacting spin chains II: the finite size effects, JHEP 08 (2013) 128 [arXiv:1302.3350] [INSPIRE].

[63] T. Bargheer, N. Beisert and F. Loebbert, Boosting Nearest-Neighbour to Long-Range Integrable Spin Chains, J. Stat. Mech. (2008) L11001 [arXiv:0807.5081] [INSPIRE].

[64] T. Bargheer, N. Beisert and F. Loebbert, Long-Range Deformations for Integrable Spin Chains, J. Phys. A 42 (2009) 285205 [arXiv:0902.0956] [InSPIRE].

[65] M. Gaudin, B.M. McCoy and T.T. Wu, emphNormalization sum for the Bethe's hypothesis wave functions of the Heisenberg-Ising chain, Phys. Rev. D 23 (1981) 417.

[66] V. Korepin, Calculation of norms of Bethe wave functions, Commun. Math. Phys. 86 (1982) 391 [InSPIRE].

[67] S. Frolov and A.A. Tseytlin, Semiclassical quantization of rotating superstring in $A d S_{5} \times S^{5}$, JHEP 06 (2002) 007 [hep-th/0204226] [INSPIRE].

[68] R. Baxter, Corner transfer matrices of the eight-vertex model. 1. Low-temperature expansions and conjectured properties, J. Statist. Phys. 15 (1976) 485.

[69] H.B. Thacker, Corner Transfer Matrices and Lorentz Invariance on a Lattice, Physica 18D (1986) 348.

[70] H. Itoyama and H. Thacker, Lattice Virasoro Algebra and Corner Transfer Matrices in the Baxter Eight Vertex Model, Phys. Rev. Lett. 58 (1987) 1395 [INSPIRE].

[71] M. Tetelman, Lorentz group for two-dimensional integrable lattice systems, Sov. Phys. JETP. $\mathbf{5 5}$ (1982) 306.

[72] E. Sklyanin, Quantum inverse scattering method. Selected topics, hep-th/9211111 [INSPIRE].

[73] F. Loebbert, Integrable Spin Chains in $\mathcal{N}=4$ super Yang-Mills Theory, Ph.D. Thesis, Max-Planck-Institut für Gravitationsphysik (Albert-Einstein-Institut), Potsdam-Golm Germany (2010). 
[74] V. Inozemtsev, Integrable Heisenberg-van Vleck chains with variable range exchange, Phys. Part. Nucl. 34 (2003) 166 [hep-th/0201001] [INSPIRE].

[75] F. Haldane, Exact Jastrow-Gutzwiller resonating valence bond ground state of the spin 1/2 antiferromagnetic Heisenberg chain with $1 / r^{2}$ exchange, Phys. Rev. Lett. 60 (1988) 635 [INSPIRE].

[76] B. Sriram Shastry, Exact solution of an $S=1 / 2$ Heisenberg antiferromagnetic chain with long ranged interactions, Phys. Rev. Lett. 60 (1988) 639 [INSPIRE].

[77] N. Beisert, C. Kristjansen and M. Staudacher, The Dilatation operator of conformal $N=4$ super Yang-Mills theory, Nucl. Phys. B 664 (2003) 131 [hep-th/0303060] [INSPIRE].

[78] G. Arutyunov, S. Frolov and M. Staudacher, Bethe ansatz for quantum strings, JHEP 10 (2004) 016 [hep-th/0406256] [INSPIRE].

[79] N. Beisert, R. Hernandez and E. Lopez, A crossing-symmetric phase for $A d S_{5} \times S^{5}$ strings, JHEP 11 (2006) 070 [hep-th/0609044] [INSPIRE].

[80] N. Beisert, B. Eden and M. Staudacher, Transcendentality and Crossing, J. Stat. Mech. (2007) P01021 [hep-th/0610251] [INSPIRE].

[81] F. Loebbert, Recursion Relations for Long-Range Integrable Spin Chains with Open Boundary Conditions, Phys. Rev. D 85 (2012) 086008 [arXiv:1201.0888] [INSPIRE].

[82] N. Beisert, L. Fiévet, M. de Leeuw and F. Loebbert, Integrable Deformations of the XXZ Spin Chain, J. Stat. Mech. (2013) P09028 [arXiv:1308.1584] [INSPIRE].

[83] N.A. Slavnov, Calculation of scalar products of wave functions and form factors in the framework of the algebraic Bethe ansatz, Theor. Math. Phys. 79 (1989) 502.

[84] I. Kostov and Y. Matsuo, Inner products of Bethe states as partial domain wall partition functions, JHEP 10 (2012) 168 [arXiv:1207.2562] [INSPIRE].

[85] E. Bettelheim and I. Kostov, Semi-classical analysis of the inner product of Bethe states, arXiv: 1403.0358 [INSPIRE].

[86] D. Serban, unpublished.

[87] V. Kazakov, A. Marshakov, J. Minahan and K. Zarembo, Classical/quantum integrability in AdS/CFT, JHEP 05 (2004) 024 [hep-th/0402207] [INSPIRE].

[88] B. Sutherland, Low-Lying Eigenstates of the One-Dimensional Heisenberg Ferromagnet for any Magnetization and Momentum, Phys. Rev. Lett. 74 (1995) 816 [INSPIRE].

[89] I. Kostov, Classical Limit of the Three-Point Function of $N=4$ Supersymmetric Yang-Mills Theory from Integrability, Phys. Rev. Lett. 108 (2012) 261604 [arXiv:1203.6180] [INSPIRE].

[90] C.G. Callan Jr. et al., Quantizing string theory in $A d S_{5} \times S^{5}$ : Beyond the pp wave, Nucl. Phys. B 673 (2003) 3 [hep-th/0307032] [INSPIRE].

[91] J.A. Minahan, The SU(2) sector in AdS/CFT, Fortsch. Phys. 53 (2005) 828 [hep-th/0503143] [INSPIRE].

[92] N. Beisert, V. Kazakov and K. Sakai, Algebraic curve for the $\mathrm{SO}(6)$ sector of AdS/CFT, Commun. Math. Phys. 263 (2006) 611 [hep-th/0410253] [INSPIRE].

[93] T. Bargheer, N. Beisert and F. Loebbert, Exact Superconformal and Yangian Symmetry of Scattering Amplitudes, J. Phys. A 44 (2011) 454012 [arXiv:1104.0700] [InSPIRE]. 